\title{
CSI 2264: Investigating rotation and its connection with disk accretion in the young open cluster NGC 2264 $\star \star \star \star$
}

\author{
L. Venuti ${ }^{1,2}$, J. Bouvier ${ }^{2}$, A. M. Cody ${ }^{3}$, J. R. Stauffer ${ }^{4}$, G. Micela ${ }^{1}$, L. M. Rebull ${ }^{4}$, S. H. P. Alencar ${ }^{5}$, A. P. Sousa ${ }^{5}$, \\ L. A. Hillenbrand ${ }^{6}$, and E. Flaccomio ${ }^{1}$
}
${ }^{1}$ Istituto Nazionale di Astrofisica, Osservatorio Astronomico di Palermo G. S. Vaiana, Piazza del Parlamento 1, 90134 Palermo, Italy e-mail: lvenuti@astropa.unipa.it
2 Univ. Grenoble Alpes, CNRS, IPAG, 38000 Grenoble, France
${ }^{3}$ NASA Ames Research Center, Kepler Science Office, Mountain View, CA 94035, USA
${ }^{4}$ Spitzer Science Center, California Institute of Technology, 1200 E California Blvd., Pasadena, CA 91125, USA
5 Departamento de Física - ICEx - UFMG, Av. Antônio Carlos, 6627, 30270-901 Belo Horizonte, MG, Brazil
6 Astronomy Department, California Institute of Technology, Pasadena, CA 91125, USA

Received 16 August 2016 / Accepted 25 October 2016

\begin{abstract}
Context. The low spin rates measured for solar-type stars at an age of a few Myr ( $\sim 10 \%$ of the break-up velocity) indicate that some mechanism of angular momentum regulation must be at play in the early pre-main sequence. This may be associated with magnetospheric accretion and star-disk interaction, as suggested by observations that disk-bearing objects (CTTS) are slower rotators than diskless sources (WTTS) in young star clusters.

Aims. We characterize the rotation properties for members of the star-forming region NGC 2264 ( $3 \mathrm{Myr})$ as a function of mass, and investigate the accretion-rotation connection at an age where about $50 \%$ of the stars have already lost their disks.

Methods. We examined a sample of 500 cluster members ( $40 \%$ with disks, $60 \%$ without disks), distributed in mass between $\sim 0.15$ and $2 M_{\odot}$, whose photometric variations were monitored in the optical for 38 consecutive days with the CoRoT space observatory. Light curves were analyzed for periodicity using three different techniques: the Lomb-Scargle periodogram, the autocorrelation function and the string-length method. Periods were searched in the range between 0.17 days (i.e., $4 \mathrm{~h}$, twice the data sampling adopted) and 19 days (half the total time span). Period detections were confirmed using a variety of statistical tools (false alarm probability, Q-statistics), as well as visual inspection of the direct and phase-folded light curves.

Results. About $62 \%$ of sources in our sample were found to be periodic; the period detection rate is $70 \%$ among WTTS and $58 \%$ among CTTS. The vast majority of periodic sources exhibit rotational periods shorter than $13 \mathrm{~d}$. The period distribution obtained for the cluster consists of a smooth distribution centered around $P=5.2 \mathrm{~d}$ with two peaks, located respectively at $P=1-2 \mathrm{~d}$ and at $P=3-4 \mathrm{~d}$. A separate analysis of the rotation properties for CTTS and WTTS indicates that the $P=1-2 \mathrm{~d}$ peak is associated with the latter, while both groups contribute to the $P=3-4 \mathrm{~d}$ peak. The comparison between CTTS and WTTS supports the idea of a rotationaccretion connection: their respective rotational properties are statistically different, and CTTS rotate on average more slowly than WTTS. We also observe that CTTS with the strongest signatures of accretion (largest UV flux excesses) tend to exhibit slow rotation rates; a clear dearth of fast rotators with strong accretion signatures emerges from our sample. This connection between rotation properties and accretion traced via UV excess measurements is consistent with earlier findings, revealed by IR excess measurements, that fast rotators in young star clusters are typically devoid of dusty disks. On the other hand, WTTS span the whole range of rotation periods detected across the cluster. We also investigated whether the rotation properties we measure for NGC 2264 members show any dependence on stellar mass or on stellar inner structure (radiative core mass to total mass ratio). No statistically significant correlation emerged from our analysis regarding the second issue; however, we did infer some evidence of a period-mass trend, lower-mass stars spinning on average faster than higher-mass stars, although our data did not allow us to assess the statistical significance of such a trend beyond the $10 \%$ level.

Conclusions. This study confirms that disks impact the rotational properties of young stars and influence their rotational evolution. The idea of disk-locking, recently tested in numerical models of the rotational evolution of young stars between 1 and $12 \mathrm{Myr}$, may be consistent with the pictures of rotation and rotation-accretion connection that we observe for the NGC 2264 cluster. However, the origin of the several substructures that we observe in the period distribution, notably the multiple peaks, deserves further investigation.
\end{abstract}

Key words. accretion, accretion disks - stars: low-mass - stars: pre-main sequence - stars: rotation -

stars: variables: T Tauri, Herbig Ae/Be - open clusters and associations: individual: NGC 2264

\section{Introduction}

* Based on observations obtained with the CoRoT space telescope, and with the wide-field imager MegaCam at the Canada-France-Hawaii Telescope (CFHT).

$\star \star$ Table F.1 is also available at the CDS via anonymous ftp to cdsarc.u-strasbg. fr (130.79.128.5) or via

http://cdsarc.u-strasbg.fr/viz-bin/qcat?J/A+A/599/A23
In spite of a substantial effort devoted to the subject over recent decades, the evolution of stellar angular momentum during the pre-main sequence (PMS) remains a somewhat controversial issue. The so-called angular momentum problem (e.g., Bodenheimer 1995) is a long-standing conundrum in star 
formation theories. At an age of a few Myr, low-mass solar type stars (T Tauri stars, TTS; Joy 1945) are known from observations to have a spin rate of merely a fraction of their break-up velocity (e.g., Vogel \& Kuhi 1981; Bouvier et al. 1986; Hartmann \& Stauffer 1989). However, if their early rotational evolution was simply governed by conservation of angular momentum as these objects contract toward the zero age main sequence (ZAMS), by an age of $\lesssim 1$ Myr they ought to rotate much faster. This indicates that some mechanism of angular momentum regulation must be at play during the early PMS that effectively counteracts the spin-up effect linked to stellar contraction. In addition, various observational studies of rotation rates in young stellar clusters (e.g., Edwards et al. 1993; Bouvier et al. 1993; Herbst et al. 2002; Lamm et al. 2005; Littlefair et al. 2010; Henderson \& Stassun 2012; Affer et al. 2013) have reported evidence of statistically distinct rotational behaviors between classical T Tauri stars (CTTS; Herbig 1962) and weak-lined T Tauri stars (WTTS; Herbig \& Bell 1988) within the same region. The former, which are young stellar objects (YSOs) still interacting with an active accretion disk, rotate on average more slowly than WTTS, which are more evolved young stars with no signatures of circumstellar material. These results indicate that angular momentum regulation in YSOs is intimately connected with the star-disk interaction.

The main idea behind the current model of disk accretion in T Tauri stars was first examined in Königl (1991). Based on the formalism developed in Ghosh \& Lamb (1978) for neutron stars, the author suggested that disks around TTS do not reach down to the stellar surface, but are truncated at a distance $R_{\mathrm{T}}$ of a few stellar radii from the central source by the strong magnetic field of the star $(\sim 1 \mathrm{kG}$ at the stellar surface). The accretion of matter from the inner disk to the star therefore occurs in a magnetically controlled fashion: material is lifted from the inner disk and channeled along the magnetic field lines, forming accretion columns that impact the stellar surface at near freefall velocities and thus generate hot shocks close to the magnetic poles. This initial picture, which assumed a stable, funnel-flow accretion process driven by a dipolar magnetosphere aligned with the rotation axis of the star, only provides a basic sketch of the far more complex and dynamic star-disk environment (see, e.g., Romanova et al. 2004; Kurosawa \& Romanova 2013). Nevertheless, the concept of magnetospheric accretion now defines the widely accepted paradigm for disk accretion in TTS, and finds strong support in its capability to explain many observational features associated with YSOs, such as the emission line profiles, large infrared and UV excesses, spectral veiling, presence of warps in the inner disk, strong photometric variability (see, e.g., Bouvier et al. 2007b, for a review).

In the framework of magnetically controlled star-disk interaction, several scenarios have been proposed to solve the angular momentum problem. Königl (1991) suggested that the magnetospheric star-disk coupling may effectively lock the star to the disk. Magnetic field lines threading the disk in the region between $R_{\mathrm{T}}$ and the co-rotation radius $R_{\mathrm{C}}$ (where the orbital velocity in the disk is higher than the angular velocity of the star) transmit a spin-up torque to the star; this is balanced by the spindown torque ensuing from magnetosphere-disk coupling beyond $R_{\mathrm{C}}$. The result is a braking action on the star. A somewhat different mechanism was proposed in Shu et al. (1994), who identified the main source of angular momentum removal from the system in magnetocentrifugally driven winds launched from the diskplane at distances $r>R_{\mathrm{C}}$. Inside $R_{\mathrm{C}}$, near-corotation of disk material with the star is enforced by the strong magnetic field. Both models assume that the dipolar component of the stellar magnetic field, which dominates the star-disk interaction (it decays more slowly with distance from the star than higherorder components), has a strength of a few $\mathrm{kG}$. However, recent studies suggest that the dipole intensity may be a factor of 10 smaller (Gregory et al. 2012). This may imply that the actual stellar dipole is not strong enough to act as an efficient braking source on the star. Alternative models of star-disk interaction have suggested that other mechanisms, such as accretionpowered stellar winds (Matt \& Pudritz 2005) or magnetospheric ejections of material (Zanni \& Ferreira 2013), may play a more decisive role in extracting angular momentum from the systems.

Although the idea of disk-locking in PMS stars has been standing since the early ' 90 s, evidence for this mechanism is still controversial. One consequence of the disk-locking scenario is that, once the disk accretion phase is over, a young star is relieved of the braking effect and can start to spin up freely as it contracts. Therefore, from an observational perspective, an association between the measured rotation periods for young stars and the presence/absence of disk accretion is to be expected if the model is valid. Systematic surveys of rotation in young clusters are of utmost interest to shed some light on these issues: intra-cluster studies enable investigations of the link between rotational properties and other stellar properties and disk indicators; exploring how the distribution of rotation periods varies between clusters of different age traces the evolution of angular momentum in the PMS. The recent review of Bouvier et al. (2014) on the matter well illustrates the current state of the debate. Evidence of statistically distinct rotational behaviors for WTTS and CTTS, reported in several studies, is not supported by others; in some cases, contrasting conclusions are drawn by different authors on the same clusters. External factors such as observational biases or sample completeness, or physical effects such as differing behaviors in different mass ranges, may play a role in this ambiguity.

The typical approach to explore a connection between rotation and disks in young clusters consists of combining optical monitoring surveys, to measure the rotational periods, with near- and mid-IR photometry, to detect a flux excess linked to thermal emission by dust in the stellar surroundings. This approach has been pursued, for instance, in Edwards et al. (1993) for a composite sample of T Tauri stars, Xiao et al. (2012) in Taurus, Herbst et al. (2002) for the Orion Nebula Cluster (ONC), Rebull et al. (2006) in Orion; these studies have shown a statistical association between the amount of IR excess and rotation, in the sense that YSOs with large IR excesses tend to be slow rotators, while young stars with little or no IR excess are spread over a broader range of periods, including both slow and fast rotators. While being indicative of dusty disks, the IR excess diagnostics does not enable direct assessment of whether an accretion process is actually ongoing in the system and at which rate mass is being transferred from the inner disk to the star. This is most directly investigated by detecting and measuring the UV flux excess produced in the accretion shock at the stellar surface. A comparison between UV excess measurements and spin rates derived for large populations of young stars is therefore of great interest to investigate the impact of different accretion regimes on the rotational properties of these sources (see, e.g., Rebull 2001; Makidon et al. 2004). The possibility of an association between UV excess and rotation in young stars was tested by Fallscheer \& Herbst (2006) for a sample of about 100 objects in the 3 Myr-old cluster NGC 2264. Sources with active accretion disks, characterized by a flux excess in the $U$-band and thus smaller (bluer) $U-V$ colors than non-accreting objects, were shown to be slow rotators, whereas fast rotators in the 
sample did not exhibit significant emission in the $U$-band above the photospheric level. This result supports the view that the angular momentum regulation in TTS is related to the process of mass accretion from the disk. As underlined by the authors, large samples of objects with UV excess measurements and rotational periods are needed to explore in detail this rotation-accretion connection. Unfortunately, the challenging and time-consuming nature of UV observations has often limited the use of this diagnostics in studies of young stars and of their evolution.

In this paper, we present a new study of rotation and of its connection with accretion disks across the young open cluster NGC 2264. This investigation has been conducted in the framework of the "Coordinated Synoptic Investigation of NGC 2264" project (CSI 2264; Cody et al. 2014); this consisted of a multisite exploration of YSOs variability in the NGC 2264 cluster, from the X-ray domain to UV, optical and mid-IR wavelengths, on timescales from <hours to several weeks. About fifteen observing sites, both space-borne (e.g., the Spitzer and CoRoT space observatories) and ground-based (e.g., the Canada-FranceHawaii Telescope, CFHT), were employed in the course of the campaign. The NGC 2264 cluster has long been a benchmark for star formation studies; its young age ( $3 \mathrm{Myr})$, relative proximity (distance of about $760 \mathrm{pc}$, in the local spiral arm of the Galaxy), rich population of young stars ( 1000 known members), low average foreground extinction $\left(A_{V} \sim 0.4 \mathrm{mag}\right)$, are some of the reasons of the long-standing interest toward this star-forming region (see Dahm 2008, for a review). The study reported here is centered on the set of optical light curves obtained with the CoRoT satellite (Baglin et al. 2006), which cover a period of 38 consecutive days almost continuously, with a photometric accuracy of $\lesssim 0.01 \mathrm{mag}$. The effectiveness of CoRoT light curves for accurate period determinations was well illustrated in Affer et al. (2013), who examined the rotation properties of about 300 NGC 2264 members based on a first, 23 day-long observing run performed with the CoRoT satellite on the cluster in March 2008. In this study, we use the new, more extensive dataset from the second CoRoT run on NGC 2264 to derive accurate rotational periods for a larger sample of cluster members, both CTTS and WTTS, and combine these results with other information from the CSI 2264 campaign to investigate how the rotational properties of young stars depend on stellar parameters like mass and on the presence of disks and active accretion.

The paper is organized as follows. Section 2 provides a brief description of the CoRoT observations and of the selection of the sample of cluster members investigated in this study. Section 3 describes the methods used to derive rotational periods from the light curves and their implementation. Section 4 illustrates the different variable classes identified across the sample (introduced in more detail in Appendix A) and presents the period distribution derived for the cluster; the rotational properties of cluster members are then discussed as a function of stellar mass and of their CTTS vs. WTTS classification; some considerations on the similarity in nature of WTTS and CTTS periods are also reported. In Sect. 5, the rotation-disk connection in NGC 2264 is explored: rotational periods derived for CTTS and WTTS in our sample are combined with UV excesses from Venuti et al. (2014) to investigate the association between different accretion regimes and the rotational properties of young stars; these results are discussed with reference to the scenarios of magnetospheric accretion and disk-locking, with particular focus on the $R_{\mathrm{T}} / R_{\mathrm{C}}$ ratio estimated following theoretical predictions. In Sect. 6, the case of NGC 2264 is discussed in the context of PMS rotational evolution: its period distribution is compared to those of various clusters between 2 and $13 \mathrm{Myr}$, and their respective features are discussed as a function of mass with reference to the timescales of evolution and dispersal of protoplanetary disks; these observational results are then compared to recent semi-empirical models of rotational evolution of young stellar clusters in the presence of disk locking. Our results and conclusions are summarized in Sect. 7. Spatial coordinates, classification and rotation parameters for all sources investigated in this study are collected in Table 4, reported after the end of the main paper text. Appendix B illustrates the impact that a specific choice of bin size and/or phase may have on a histogram representation of the period distribution derived for the cluster. Cases of objects with discrepant period measurements between this study and Affer et al. (2013), objects with different periods reported in this study with respect to Cieza \& Baliber (2007), and the cases of objects periodic in the first CoRoT run (Affer et al. 2013) but aperiodic here or vice versa are discussed in Appendices C, D, and E, respectively.

\section{Observations and sample selection}

The CoRoT monitoring survey of NGC 2264 extended over 38 consecutive days from December 1, 2011 to January 9, 2012. The instrument specifications, as well as a detailed description of the observing run and of the subsequent photometry reduction, were provided in Cody et al. (2014). Observations were carried out using one of the two CCDs originally dedicated to exoplanetary science for the main scientific program of the CoRoT mission. The instrument has a field of view (FOV) of $1.3^{\circ} \times 1.3^{\circ}$, quite adequate to fit NGC 2264 in its entire spatial extent. Time series aperture photometry is downloaded from the satellite only for objects in a pre-determined list of targets in the FOV; the final CoRoT sample obtained within CSI 2264 contains about 500 sources with robust evidence of membership, 1600 candidate members and 2000 field stars. All of the NGC 2264 light curves we have utilized in producing this paper are available as part of the CSI 2264 public data release in the IRSA archive ${ }^{1}$. The website provides users to both view the light curves and to download them, either individually or as the complete set of light curves in a single tar file. In addition, all scientific data issued from the CoRoT campaign can be found at the IAS CoRoT Public Archive ${ }^{2}$.

The magnitudes of monitored objects range from 11 to 17 in the $R$-band. A time sampling of $512 \mathrm{~s}$ was adopted for most targets in the FOV, hence resulting in over 6300 datapoints per light curve. For a subset of objects, a high-cadence observing mode was adopted, with luminosity measurements every 32 s (corresponding to over 100800 datapoints along the whole observing run). Extracted light curves were preliminarily inspected and corrected for systematic effects such as isolated outliers (flagged by the CoRoT pipeline and discarded in the analysis) or abrupt flux discontinuities due to detector temperature jumps (which occur in about $10 \%$ of light curves in our sample at the same observing epochs).

The sample of cluster members investigated in this study was built following primarily the member list provided in Venuti et al. (2014). Membership and classification (WTTS vs. CTTS) criteria are listed in that paper and comprise photometric or spectroscopic $\mathrm{H} \alpha$, X-ray emission, radial velocity and UV/IR excess diagnostics. The CoRoT counterparts of these sources were identified as their closest match within a radius of 1 arcsec around their spatial coordinates. The sample of members studied

\footnotetext{
http://irsa.ipac. caltech.edu/data/SPITZER/CSI2264/ http://idoc-corot.ias.u-psud.fr/
} 


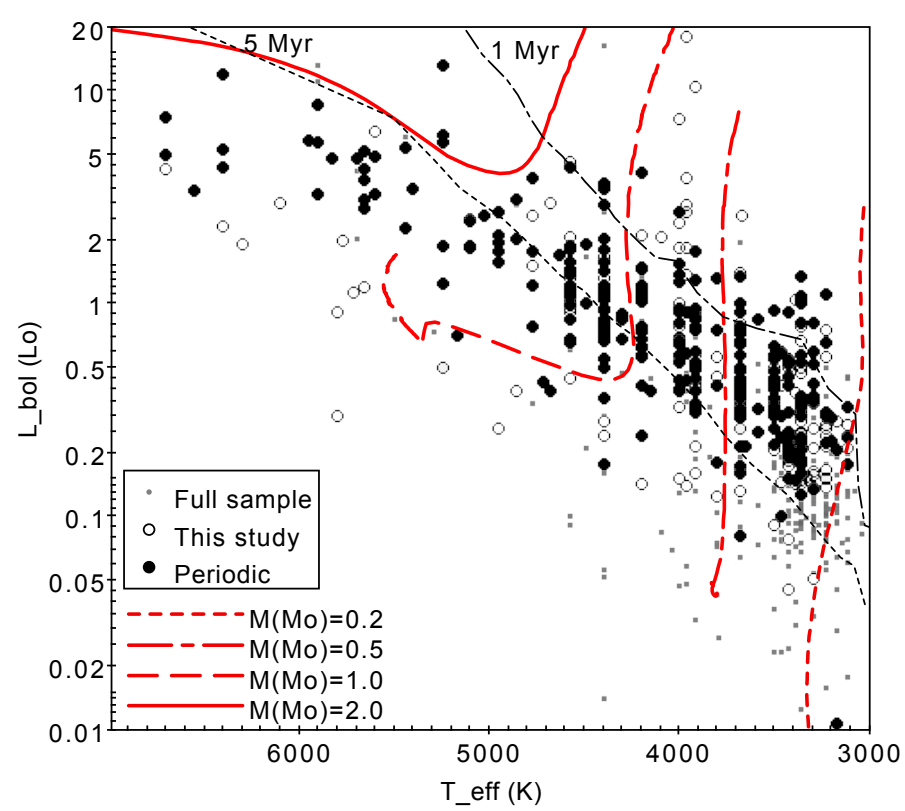

Fig. 1. Properties of NGC 2264 members considered in this study (open and filled circles) are compared to those of the full sample of NGC 2264 members available from CSI 2264 (grey dots). Filled circles identify the periodic sources emerging from the analysis in Sect. 3. Mass tracks shown on the diagram and isochrones at $1 \mathrm{Myr}$ (dash-dotted line) and 5 Myr (dotted line) are from Siess et al.'s (2000) models.

in Venuti et al. (2014, Table 2) includes 757 objects; among these, only 433 have a CoRoT counterpart from the CSI 2264 observing run. A good fraction of the sources not matched in the CoRoT sample are fainter than $R=17$. Additional members, not included in Venuti et al.'s (2014) sample (mainly objects that fell outside the FOV probed in that paper, or brighter than the magnitude range explored), were selected from the CSI 2264 master catalog ${ }^{3}$ of the region, upon the condition of being flagged as "very likely NGC 2264 member" (i.e., MEM = 1; see Cody et al. 2014). The final sample of members thus selected for the present study of rotation comprises $500^{4}$ objects, distributed in mass between $\sim 0.1$ and $2 M_{\odot}$; the ratio of disk-bearing (CTTS) to diskfree (WTTS) members is of about $40 \%$ to $60 \%$.

In Fig. 1, the properties of the subsample of objects examined in this study (periodic or aperiodic as resulting from the analysis in Sect. 3) are compared to those of the full NGC 2264 population on a H-R diagram. Effective temperatures $T_{\text {eff }}$ were assigned to each object based on their spectral type SpT and the SpT- $T_{\text {eff }}$ conversion scale provided in Cohen \& Kuhi (1979, see also Luhman et al. 2003); bolometric luminosities $L_{\text {bol }}$ were derived from the dereddened 2MASS $J$-band magnitudes, us-

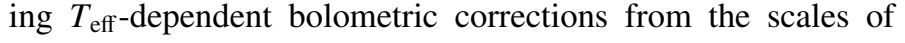

\footnotetext{
3 Available at http://irsa.ipac.caltech.edu/data/SPITZER/ CSI2264/

4 In the process of members selection, we rejected the object CSIMon000661, that had been classified as member in Cody et al. (2014) and Venuti et al. (2014). The reasons for its exclusion are the following: this star is located beyond the periphery of the molecular cloud in an RADec diagram of the region; the CoRoT light curve amplitude for the source is of $\sim 0.02 \mathrm{mag}$, significantly lower than the typical amount of variability measured for these young stars; VLT/FLAMES data acquired for this object show no signs of lithium absorption, which suggests that this star is more evolved; the previous classification as cluster member was based on Fürész et al.'s (2006) radial velocity survey of the cluster, but the specific measurement for this object stood about 3-4 $\sigma$ away from the typical cluster locus.
}

Pecaut \& Mamajek (2013) and Bessell et al. (1998). The reader is referred to Venuti et al. (2014) for further details.

It can be seen that objects appear to be spread over a broad range of $L_{\mathrm{bol}}$ at any given $T_{\mathrm{eff}}$. At first sight, this would indicate a significant age/evolutionary spread among cluster members, although a number of recent studies have stressed how separate effects such as individual accretion histories (Baraffe et al. 2009) or observational uncertainties (e.g., Pecaut \& Mamajek 2016) may result in an artificial apparent age spread on a H-R diagram. Notably, Fig. 1 shows a population of about 25 objects, classified as cluster members and with no detectable periodic behavior, that define a lower boundary to the distribution of the NGC 2264 population on the diagram, well below the $5 \mathrm{Myr}$ isochrone. About $55 \%$ of them are non-variable according to the Stetson's (1996) $J$-index (see Venuti et al. 2015), while the remaining $45 \%$ are variable but exhibit an irregular light curve pattern. For some objects in this group, the classification as cluster member derives from literature studies based on a limited number of parameters (e.g., radial velocity), and the small additional information available for these cases from our campaign did not enable a reassessment of the membership issue. Therefore, some of them, amounting to a small percent of the total sample, may actually be field contaminants. However, sound evidence of being a young star (presence of disk, lithium absorption) is available for other sources in this group; their position on the H-R diagram may then be affected by uncertainties on the derived stellar parameters, or by strong light attenuation by the circumstellar environment.

\section{Period search}

Due to their intense chromospheric activity, as well as magnetospheric accretion on disk-bearing objects, the surface of young stars looks far from homogeneous. In fact, a significant fraction (up to a few tenths) of it appears covered by uneven spots of different temperature relative to the photosphere. As the star rotates, different portions of the stellar surface appear on the line of sight to the observer, hence resulting in a modulation effect of the photospheric flux by surface spot seen at different phases. When objects are monitored during several rotational cycles, provided that the lifetimes of surface spots are longer than the timescales of interest, a periodicity is therefore expected to appear in the light curves, corresponding to the rotation period of the stars.

Several methods have been proposed to extract a periodic component from a time-ordered series of signal measurements. Some, more analytic, consist in decomposing the signal in waves at a given frequency; others, more empirical, are based on a comparative examination of the morphology of different segments of light curve, in units of trial period. We adopt here 3 different methods to search for periodic signals in our sample of light curves: the Lomb-Scargle periodogram (LSP), the autocorrelation function (ACF) and the string-length method (SL). A brief description and comparison of advantages and disadvantages of these methods is provided in Sect. 3.1, while details on their application to our analysis are provided in Sect. 3.2.

\subsection{Methods}

\subsubsection{Lomb-Scargle periodogram}

This approach (Scargle 1982; Horne \& Baliunas 1986) is a revised version of the discrete Fourier transform method, applicable to datasets with uneven temporal sampling and invariant to a shift of time origin. The method is equivalent to least-squares 

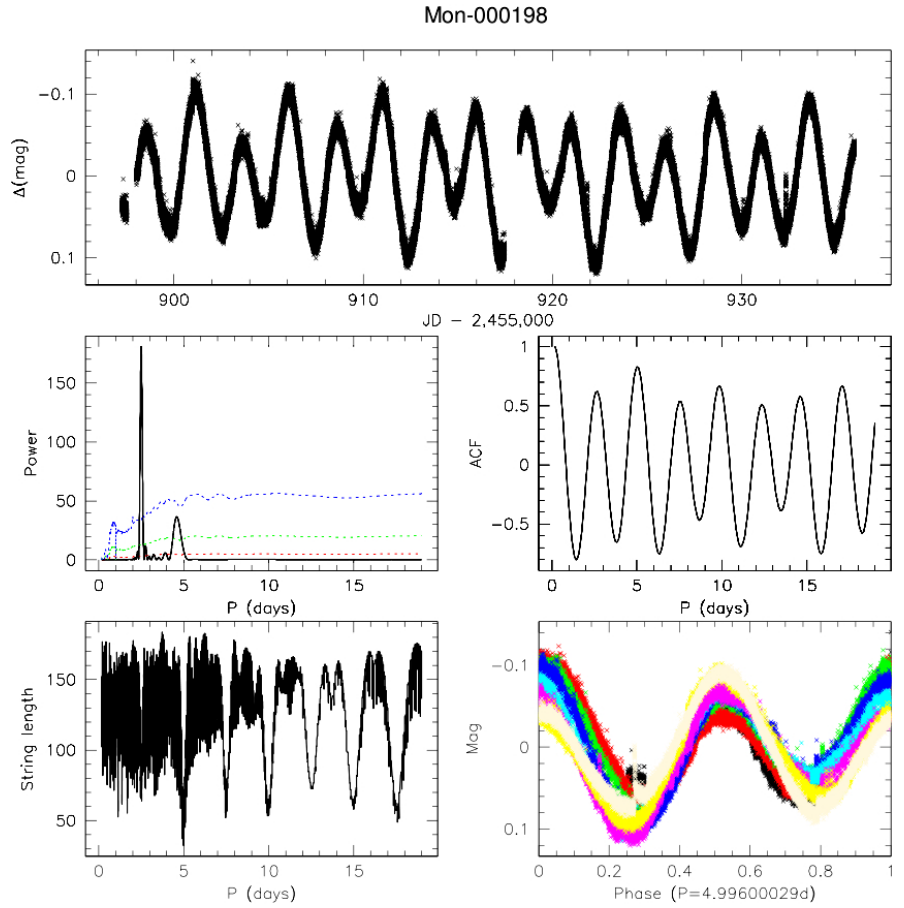

Fig. 2. Period analysis for the object with multiple spots CSIMon000198: original light curve after removal of spurious points in the upper panel, periodogram in the middle left panel, auto-correlation function in the middle right panel, string-length method in the lower left panel, phased light curve with the final period in the lower right panel. Red, green and blue dotted curves superimposed over the periodogram in the middle left panel correspond respectively to the mean, mean $+3 \sigma$ and mean $+10 \sigma$ periodogram expected in case the mag fluctuations about the mean were due to random noise. Different colors in the lower right panel correspond to different rotational cycles. Due to the nearly symmetric shape of the main periodic feature around a less deep minimum at about the half-period, this last is erroneously detected as the highest power (best rotational period) when using the periodogram analysis; on the other hand, the correct periodicity is indicated by both the other methods.

fitting of sinusoidal waves at a test frequency $\omega$ to the observed light curve: the power spectrum of the light curve is reconstructed by varying the test frequency $\omega$ in the range of investigation.

The LSP method ensures more accurate period measurement than ACF and SL, as it is less sensitive to spurious points and long-term trends; in addition, it provides a straightforward estimate of the uncertainty on the derived periodicity, by measuring the Gaussian width of the highest peak in the periodogram. On the other hand, the explicit assumption that the periodic luminosity component can be described in terms of sine curves may contrast with the actual light curve shape observed for these young stellar objects. Moreover, this analytic approximation may lead to incorrect period identifications when the folded light curve is nearly symmetrical with respect to phase $\phi=0.5$, as illustrated by the case in Fig. 2.

\subsubsection{Auto-correlation function}

Contrary to the LSP method, the ACF method (Box \& Jenkins 1976; McQuillan et al. 2013) does not introduce any assumptions on the shape of the light curve. The method consists in exploring a range of trial periods and computing, for each of them, the autocorrelation coefficient $r_{k}$ of the light curve at lag $k$, where $k$ is the tested period $P$ in units of time cadence $\Delta t(P=k \Delta t)$ :

$r_{k}=\frac{\sum_{i=1}^{N-k}\left(m_{i}-\bar{m}\right)\left(m_{i+k}-\bar{m}\right)}{\sum_{i=1}^{N}\left(m_{i}-\bar{m}\right)^{2}}$,

where $m_{i}$ is the magnitude at time $t_{i}, m_{i+k}$ is the magnitude at time $t_{i}+P, \bar{m}$ is the average light curve magnitude and $N$ is the total number of light curve points (equally spaced in time with step $\Delta t$ ). The auto-correlation function is reconstructed by plotting $r_{k}$ as a function of $P$. When the $P$ value tested matches the actual periodicity of the light curve, similar behaviors relative to the typical luminosity state $\bar{m}$ are expected at epochs $i$ and $i+k$, hence resulting in large values of $r_{k}$ and a maximum in the ACF; conversely, small values of $r_{k}$ are found if matched epochs $i$ and $i+k$ are out-of-phase.

By virtue of its direct reference to the actual shape of the light curve, the ACF technique allows more reliable identification of the peak corresponding to the true periodicity (as shown in Fig. 2); however, the need for an even time binning and for adopting a $\Delta P$ step for period investigation that is the same as (or a multiple of) the light curve $\Delta t$ reduces the accuracy of the extracted period value compared with the value of the corresponding peak in the periodogram.

\subsubsection{String-length method}

As with ACF, the SL method (Dworetsky 1983) does not introduce any assumptions on the shape of the light curve. The method explores a range of trial periods; for each $P$, light curve points are ordered in phase and the string-length $L$ is defined as the sum of the lengths of line segments that connect successive points of the phased light curve on the $(m, \phi)$ diagram:

$$
\begin{aligned}
L= & \sum_{i=1}^{N-1} \sqrt{\left(m_{i}-m_{i-1}\right)^{2}+\left(\phi_{i}-\phi_{i-1}\right)^{2}} \\
& +\sqrt{\left(m_{1}-m_{N}\right)^{2}+\left(\phi_{1}-\phi_{N}+1\right)^{2}}
\end{aligned}
$$

A preliminary rescaling of the observed magnitude range to the phase range is needed in order to assign equal weight to variations in the two variables for the computation of $L$. The best period $P$ is the one that minimizes the value of $L$, while large $L$ values will be found when the light curve is phased with an arbitrary period, hence resulting in a scattered cloud of points with no specific pattern on the phase diagram.

This technique combines the advantages of being conceptually straightforward, directly related to the actual variability pattern of the light curve, and of being applicable to any temporal sampling; due to the greater sensitivity to spurious points and long-term trends, the overall SL curve as a function of $P$ tends to be more noisy, notably at lower period values.

\subsection{Implementation}

A preliminary light curve "cleaning" routine was performed to reject all points with flag $\neq 0$ from the CoRoT reduction pipeline ${ }^{5}$. A $10 \sigma$-clipping selection was subsequently performed to discard isolated discrepant points.

Resulting light curves were then rebinned to $2 \mathrm{~h}$. This time step choice was on one side motivated by the $1.7 \mathrm{~h}$-long orbital period of the CoRoT satellite, smoothed out in this new data

5 The meaning of different flag values is detailed in the manual on CoRoT N2 data stored at http://idoc-corot.ias.u-psud. fr 
binning (see also Affer et al. 2012, 2013); this also represents a suitable choice with respect to computation efficiency. We computed the expected break-up velocity $\mathcal{V}^{6}$ for all sources in our sample with estimated mass $M_{\star}$ and radius $R_{\star}$ from Venuti et al. (2014; 86\%), and ascertained that this would result in minimum rotational periods longer than $2 \mathrm{~h}$ for the vast majority of objects. The typical break-up period we estimated across our sample is 0.5 days; less than $1 \%$ of the considered objects have $\mathcal{V}>2 \pi R_{\star} / 2 h$, while about $6 \%$ have $\mathcal{V}>2 \pi R_{\star} / 4 h$ (where $4 \mathrm{~h}$ is the smallest period that can be investigated following the time step definition). Therefore, we assume that no significant bias on the detection of high-frequency periodicities is potentially introduced when performing the data rebinning to $2 \mathrm{~h}$. Nevertheless, we did adopt a smaller temporal bin and inspected the light curves for periods shorter than $4 \mathrm{~h}$ in cases where no period was found from the procedure described in the following, and when hints of a possible short periodicity were conveyed from the light curve and/or the analysis. Only for one object was a significant period shorter than $4 \mathrm{~h}$ actually detected.

Rebinned light curves are processed afterwards for period investigation. Each light curve is examined three times, using a different technique at each step, as enumerated in Sect. 3.1. Periods explored range from $4 \mathrm{~h}$ (i.e., twice the data sampling) to 19 days (i.e., half the total duration of the time series). The upper limit of the period range selection ensues from the assumption that a periodic behavior can be reliably identified if this is repeated at least twice during the monitored time; however, we did extend the range explored to longer periods in cases where the light curve and/or period diagrams obtained provided some hints of a periodicity above $19 \mathrm{~d}$. A step $\mathrm{d} P$ of $0.1 \mathrm{~h}$ is adopted to explore the period range with the LSP and the SL methods, while $\mathrm{d} P$ is set to the data cadence for the ACF method.

Period diagrams from LSP, ACF and SL methods are visually inspected and compared and the best period is selected as the one toward which the 3 diagnostic tools converge. The ACF and SL diagrams are used as the primary reference to locate the correct periodicity, for the reasons explained in Sect. 3.1; the period value is then extracted from the peak displayed in the periodogram at the position predicted from the other two methods. In case no significant features are present in the LSP diagram at this location, the period value is extracted from the SL diagram; an extensive comparison of values measured from different methods for non-ambiguous periodic variables allowed us to conclude that SL estimates are typically more accurate than ACF estimates and in very good agreement with those extracted from the LSP diagram. As illustrated in Fig. 2 and discussed in Sect. 3.1.3, peaks in the SL curve tend to be less sharp than in the ACF or the LSP. For this reason, to derive a more precise SL period, instead of taking the position of the first minimum, we extracted the position of each minimum in the diagram, then measured the distance between every pair of consecutive minima, and defined the best period as the mean of these distances.

To obtain a first indication of the significance of periodogram peaks, we adopted the following procedure (see also Affer et al. 2013; Flaccomio et al. 2005; Eaton et al. 1995). We segmented the original light curve in blocks of $12 \mathrm{~h}$, shuffled them and reassembled them in a random order. Every potential periodicity

\footnotetext{
6 The break-up velocity is defined as the tangential velocity at which the centrifugal force perceived by an element of mass at the surface of the star equals the gravitational force that keeps that mass element bound to the star: $\mathcal{V}=(2 / 3)^{1 / 2} \sqrt{G M_{\star} / R_{\star}}$, where $G$ is the gravitational constant and the factor $(2 / 3)^{1 / 2}$ accounts for the polar-to-equatorial radius ratio when the surface rotates with the critical velocity (cf. Gallet \& Bouvier 2013; Ekström et al. 2008).
}

longer than the time length of the segments is destroyed in the process, hence resulting in a test light curve where the main contribution to variability arises from photometric noise or shortlived events like flares and episodic accretion. The periodogram analysis is then performed on this "stochastic" light curve and the whole routine is iterated 1000 times. The noise periodogram is thus defined point by point as the mean power measured across the 1000 simulations at the given $\omega$ value, while the variance is used to define confidence levels above the mean. The true periodogram of the source is therefore compared to these curves in order to get an indication of the confidence associated with the period detection/non-detection. This is illustrated in the middle panel of Fig. 2. It is important to note that the confidence levels estimated with this procedure might be somewhat optimistic: irrespective of its periodic or non-periodic nature, the variability of $\mathrm{T}$ Tauri stars exhibits a characteristic coherence timescale of a few to several days (see, for instance, the analysis of variability self-correlation in non-periodic TTS presented in Percy et al. 2006). When $12 \mathrm{~h}$-long light curve segments are reshuffled, this coherence in the light curve is destroyed together with the rotational modulation; hence, the procedure may result in an underestimation of the "noise" component that affects the periodogram results.

Another statistical indicator of the degree of periodicity in the light curve is the parameter $Q$ introduced in Cody et al. (2014). This measures how well a periodic trend at the period extracted can describe the original light curve. $Q$ is defined as

$Q=\frac{\left(\mathrm{rms}_{\mathrm{resid}}^{2}-\sigma^{2}\right)}{\left(\mathrm{rms}_{\mathrm{raw}}^{2}-\sigma^{2}\right)}$,

where $\sigma$ is the photometric measurement uncertainty, $\mathrm{rms}_{\mathrm{raw}}$ is the level of rms scatter in the original light curve, and $\mathrm{rms}_{\text {resid }}$ is the level of rms scatter in the light curve after subtraction of the periodic trend (which is reconstructed by generating a smoothed phase-folded curve and overlaying it to the original light curve, repeating it once per period). $Q$ therefore measures how close the light curve points are to the systematic noise floor before and after subtraction of the phased trend from the light curve. Following the scheme of Cody et al. (2014), $Q<0.11$ indicates strictly periodic ${ }^{7}$ light curves, $0.11<Q<0.61$ corresponds to quasi-periodic ${ }^{8}$ light curves, and $Q>0.61$ indicates likely aperiodic light curves, with spurious period determination.

A careful inspection of phase-folded light curves was performed to reinforce the statistical period validation. This visual inspection is especially useful to decide on borderline cases or to select the correct periodicity in case of multiple peaks, as well as to check the accuracy of periodogram estimates (imprecise values will translate to offsets between different cycles in the phase-folded light curve). On the sole basis of individual diagrams such as Fig. 2, we sorted all objects in the sample into periodic or aperiodic; we then combined this visual classification with the results of the $Q$ statistics. In nearly $80 \%$ of the total, the results of the two selections were consistent (i.e., objects visually classified as aperiodic have $Q>0.61$ and vice versa); in the remaining cases, a final decision was taken upon further visual inspection. A few of us (L.V., J.B., A.M.C., J.R.S.) examined the

7 Light curves that exhibit stable, repeating patterns, with shapes that evolve minimally over the monitored time span.

8 Light curves that exhibit a stable period, but with varying shape and/or amplitude from one cycle to the next, or light curves where lower-amplitude, irregular flux variations are superimposed over a modulated pattern. 
Table 1. Morphology types in the light curve sample of NGC 2264 members.

\begin{tabular}{lrrrr}
\hline \hline Morphology class & Count & $\mathrm{c} / \mathrm{w}$ & $\%$ tot. & $\%$ Per \\
\hline Burster & 21 & $21 / 0$ & 4.2 & 38.1 \\
Dipper & 37 & $35 / 0$ & 7.4 & 68.6 \\
Spotted & 187 & $44 / 153$ & 37.3 & 100.0 \\
Multi-periodic & 34 & $6 / 24$ & 7.2 & 100.0 \\
Eclipsing binary & 3 & $2 / 1$ & 0.6 & 100.0 \\
Stochastic & 22 & $19 / 0$ & 4.4 & 31.6 \\
Long-timescale variable & 3 & $2 / 1$ & 0.6 & 33.3 \\
Non-variable & 82 & $20 / 40$ & 16.3 & 0.0 \\
Others* & 114 & $27 / 58$ & 22.7 & 28.7 \\
\hline
\end{tabular}

Notes. Morphology class $=$ classification of the light curve variability type; each class is briefly defined in Appendix A of this work. Count = number of objects in the sample with light curves falling in the corresponding category (a given object is here attributed to a single category, namely the one that best describes the dominant variability features of its light curve, although some cases may also exhibit traits of a different morphology class). $c / w=$ number of CTTS/number of WTTS among objects in the corresponding morphology class. \% tot. $=$ percentage of objects in the sample that fall in the corresponding morphology class. $\%$ Per $=$ percentage of objects in the corresponding morphology class which are found to be periodic. ${ }^{(*)}$ Non-sorted light curves (unclassifiable variable type or affected by, e.g., temperature jumps).

period diagrams for these objects independently, and listed what sources they thought were periodic or aperiodic. These results were then put together to assign or discard a period estimate to a given source with a certain degree of confidence.

\section{Results}

At the end of the analysis detailed in Sect. 3, a definite periodicity is detected for 309 objects, i.e., for $62 \%$ of the objects in our sample. Three of these periodic sources (CSIMon-000256, $6079,6465)$ are eclipsing binaries; another 34 exhibit more than one significant and distinct periodicity (not harmonics).

Among the 272 objects for which a single periodicity is detected in our analysis, several light curve types can be distinguished. As extensively discussed in Cody et al. (2014), the diversity in light curve morphology, very nicely highlighted in the NGC 2264 sample thanks to the accuracy and time coverage of the CoRoT dataset, likely reflects a variety of dominant physical mechanisms. Following the scheme of Cody et al. (2014), in Appendix A we provide a brief list of the main variable classes identified among cluster members analyzed in this study, and of the relevant physical processes which may dominate the detected light variations. An illustration of the various classes is shown in Fig. 3 in the main text; Table 1 provides a synthetic view of the statistical occurrence of each variable class across the NGC 2264 population, of their distribution between CTTS and WTTS, and of what fraction of objects belonging to each class is found to be periodic.

Among WTTS, a periodicity frequency of about $70 \% \pm 9 \%$ is detected; among CTTS, the frequency is of about $58 \% \pm 6 \%$. These numbers comprise both light curves that are "strictly periodic", i.e., that exhibit a stable pattern with shape that evolves minimally over the monitored span, and those that are "quasiperiodic", i.e., light curves with stable period but with changes in shape and/or amplitude from cycle to cycle (see the upper panels of Fig. 3 for a comparative illustration of the two). The fraction of NGC 2264 members, and, among these, of CTTS found to be periodic from the CoRoT sample are consistent with the fraction of periodic-to-all sources recovered in the study of Affer et al. (2013). Conversely, the fraction of WTTS with detected periodicity among the sample investigated in Affer et al. (2013) is larger $(\sim 88 \%)$ than that recovered here. This may be due to the fact that the sample of cluster members investigated here includes more stars in the low mass range than the sample investigated in Affer et al. (2013); at fainter magnitudes, the impact of photometric noise is more considerable and may blur the intrinsic modulated pattern of the light curve. Indeed, the fraction of sources that would be classified as non-variable above the noise level, based on, e.g., Stetson's (1996) J-index (see Venuti et al. 2015), is higher at fainter magnitudes. Of the 81 WTTS in our sample for which no periodicity is detected, 41 had no data in the previous CoRoT campaign, 23 had an aperiodic light curve in 2008 as well (Affer et al. 2013), 5 (not included in the study of Affer et al. 2013) exhibit a flat-line light curve in 2008, 7 were classified as periodic in Affer et al. (2013), and 3 (not included in Affer et al. 2013) show some indications of periodicity in their CoRoT light curves from 2008. The last 10 cases are discussed in detail in Appendix E.

A direct estimate to the uncertainty on period measurements from the LSP can be derived as

$\delta P=\delta v \times P^{2} \simeq 0.0096 \times P^{2}$,

where $\delta v=0.0096$ is the average sigma width of a Gaussian fit to the periodogram peak across our sample and $P$ is expressed in days. The resulting typical uncertainty amounts to $\delta P \sim 0.15$ days for periods on the order of 4 days and $\delta P \sim 1$ day for periods on the order of 10 days. This, however, appears to be very conservative, especially for longer period sources, compared to the actual accuracy we can reach thanks to the time coverage and cadence of the CoRoT light curves.

A "theoretical" estimate of the uncertainty associated with the measured periods, based on the time sampling and number of cycles occurring in the light curve, can be derived following Mighell \& Plavchan (2013). For a light curve of length $L$ and period $P$, we can compute the number $M=\operatorname{int}(L / P)$ of complete cycles contained in the light curve. The light curve will then contain $M+1$ maxima/minima, at positions $t_{1} \ldots t_{M+1}$. The light curve period can be defined as the average distance between two consecutive maxima/minima in the light curve:

$P=\frac{\left(t_{M+1}-t_{M}\right)+\left(t_{M}-t_{M-1}\right)+\cdots+\left(t_{2}-t_{1}\right)}{M}$.

If the light curve points are evenly spaced with a time step $\mathrm{d} t$, the position of each maximum/minimum has an associated uncertainty $\sigma=\mathrm{d} t / 2$. Therefore, by applying standard error propagation techniques, the uncertainty on $P$ is given by

$$
\begin{aligned}
\delta P & =\sqrt{\left(\frac{\delta t_{M+1}}{M}\right)^{2}+\left(\frac{\delta t_{M}}{M}\right)^{2}+\cdots+\left(\frac{\delta t_{1}}{M}\right)^{2}} \\
& =\sqrt{2 M\left(\frac{\sigma}{M}\right)^{2}}=\sigma \sqrt{\frac{2}{M}}
\end{aligned}
$$

where $\sigma=0.0417 \mathrm{~d}$ in our case. This corresponds to an uncertainty of $0.02 \mathrm{~d}$ for periods of $4 \mathrm{~d}$ and of $0.03 \mathrm{~d}$ for periods of $10 \mathrm{~d}$. Uncertainties estimated from Eq. (5) are slightly larger than those derived from Eq. (4) for periods shorter than $1 \mathrm{~d}$, and significantly smaller for periods of several days. The reasoning adopted to derive Eq. (6) assumes that the light curve is strictly periodic, so that the only uncertainty on the position of 

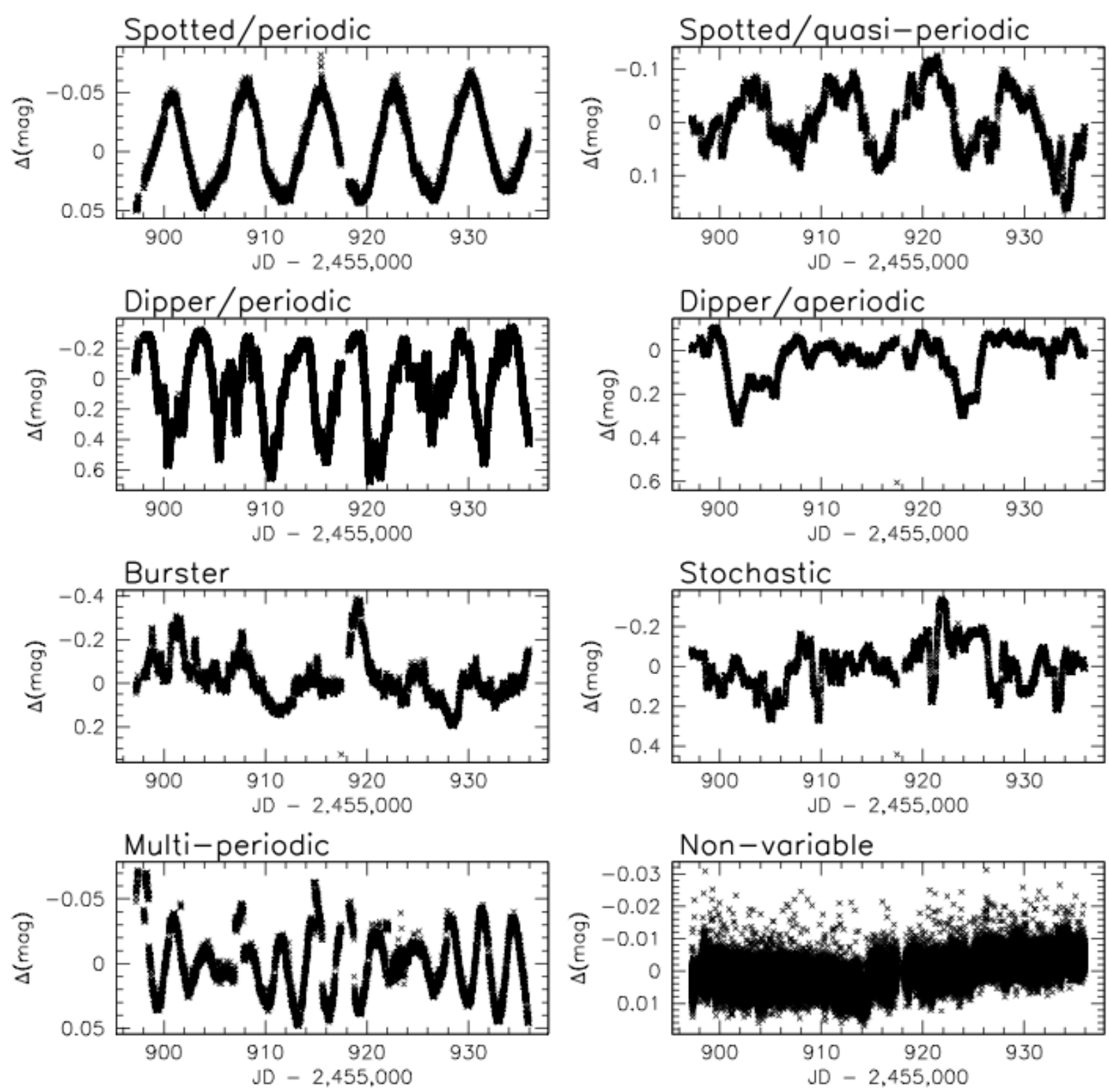

Fig. 3. Examples of the different morphological classes of light curves identified among the population of NGC 2264, in the optical dataset acquired with CoRoT. The objects illustrated are, from top to bottom, from left to right: CSIMon-000954 (strictly periodic), CSIMon-001167 (quasi-periodic), CSIMon-000660 (periodic dipper), CSIMon-000126 (aperiodic dipper), CSIMon-000567 (burster), CSIMon-000346 (stochastic), CSIMon-000967 (multi-periodic), CSIMon-000069 (non-variable).

maxima/minima derives from the non-sampled time interval between two consecutive light curve points. However, this is not the case for the majority of light curves in our sample. Therefore, for illustration purposes we will use here error bars derived from Eq. (6), but we note that realistic uncertainties on the measured periods probably lie between the estimates from Eq. (6) and those from Eq. (4).

The period measurements derived in this study are reported in Table 4. In Fig. 4, these values are compared to the results of the similar investigation performed in Affer et al. (2013) from the first CoRoT run on NGC 2264, and those reported in Cieza \& Baliber (2007), which in turn refer to the studies performed in the optical by Lamm et al. (2004) and Makidon et al. (2004).

The intersection between the sample of periodic sources with single periodicity found in this study and that of periodic variables listed in Affer et al. (2013) consists of 117 sources. Among these, $16(14 \%)$ have different period estimates between the two studies (i.e., the estimate of period derived here for these objects is not consistent with Affer et al.'s estimate within the error bar associated with our measurements following the prescription of Eq. (4)). These cases are individually illustrated and discussed in Appendix C; several of them are harmonics at half or double period.

Similar considerations are reached when comparing our period estimates to those reported in Cieza \& Baliber (2007). The common sample in this case comprises 145 objects, out of which $24(17 \%)$ have different period estimates in the two studies. A significant fraction of these outliers with respect to the equality line in Fig. 4 (right panel) lies along a horizontal line at $P($ Cieza $\&$ Baliber 2007$) \sim 1 \mathrm{~d}$; the corresponding periods derived in this study range from about $1 \mathrm{~d}$ to $12 \mathrm{~d}$. As Cieza \& Baliber's (2007) period distribution is based on photometric measurements performed from the ground, these 1-day 

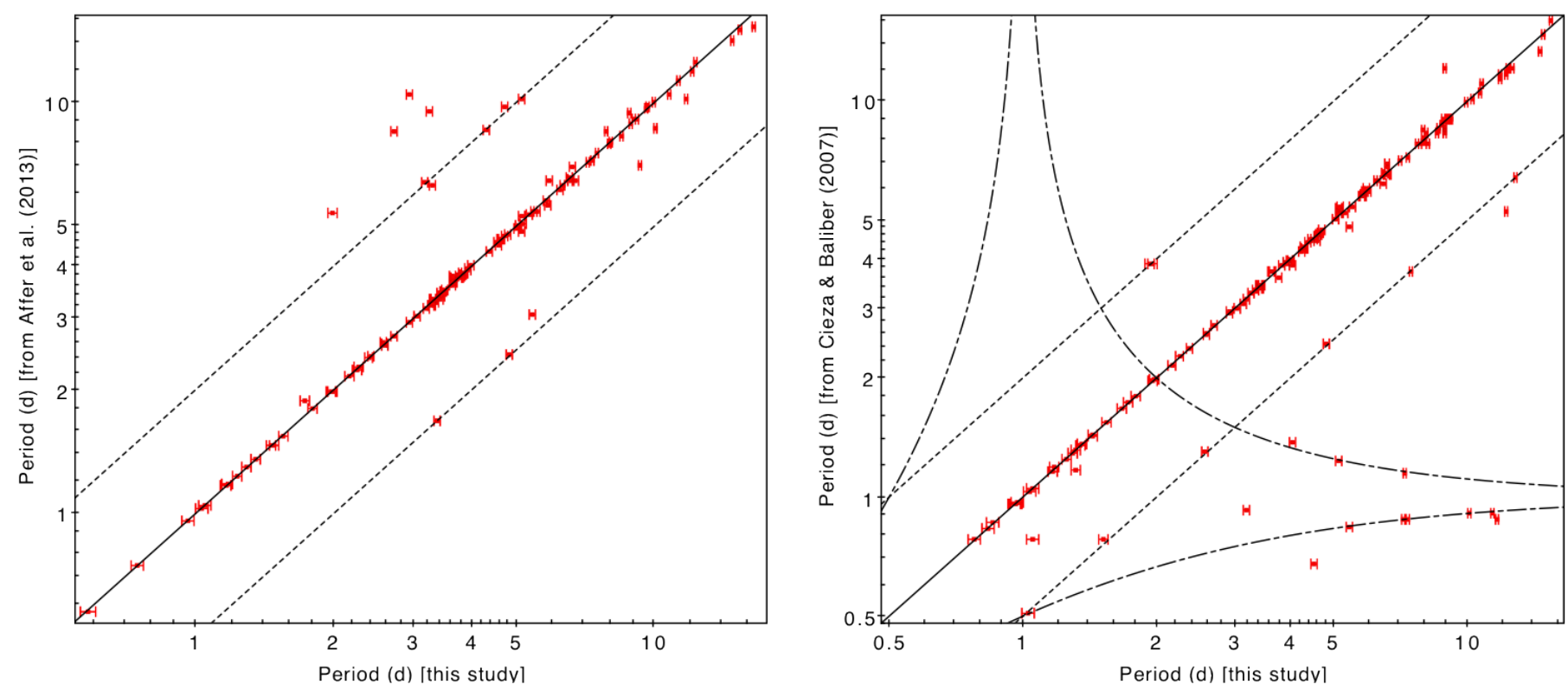

Fig. 4. Comparison of the period values derived in this study with those obtained in the study of Affer et al. (2013; left panel; 117 sources depicted) and those reported in Cieza \& Baliber (2007; right panel; 145 sources depicted). 91\% of the periods reported in Cieza \& Baliber's (2007) sample are from Lamm et al. (2005; after Lamm et al. 2004); the remaining are from Makidon et al. (2004). The intersection between the sample shown on the left panel and that shown on the right panel of this figure amounts to 61 objects. The equality line is traced as a solid line; the half and double values lines are traced as dotted lines. The dash-dot lines trace the beat period with a $1 \mathrm{~d}$ sampling interval (e.g., Cieza \& Baliber 2006; Davies et al. 2014). Error bars along the $x$-axis are computed following Eq. (6). Multi-periodic objects are not shown on these diagrams.

periods may actually be spurious detections (aliases) linked to the limited time sampling and day-night alternance which affect observations from the ground. A similar effect was identified in Affer et al. (2013), who compared their own results to those published by Lamm et al. (2004).

In the following, we will assume that, when we detect a single photometric period in the light curve, this corresponds to the rotation rate of the star ${ }^{9}$; we will then focus on the subsample of 272 periodic sources mentioned earlier to investigate the rotation properties of cluster members. A detailed analysis of EBs (see, e.g., Gillen et al. 2014) and multi-periodic members of NGC 2264 is deferred to subsequent papers.

\subsection{Period distribution}

Figure 5 illustrates the period distribution inferred here for the NGC 2264 population. A bin size of $1 \mathrm{~d}$ was adopted for the histogram, slightly smaller than the $1.4 \mathrm{~d}$ value that would be derived when applying Freedman \& Diaconis's (1981) rule. This appears to be a reasonable choice in terms of resolution and statistics, and it is also the bin size commonly used in the literature, which enables direct comparison of the resulting period distribution with those inferred from analogous studies. Although the baseline of 38 days covered by the CoRoT light curves would enable robust detection of periodicities of up to 19 days, the vast majority of periodic cluster members have periods shorter than 13 days. This is similar to the period distribution inferred in the study of Affer et al. (2013) from the previous, 23-day long CoRoT observing run on NGC 2264.

A prominent feature of the period distribution found here is the presence of two peaks on top of a smooth distribution. The

\footnotetext{
9 Artemenko et al. (2012) argue that, for a fraction of CTTS, the periodicity that we may detect in the brightness variations is not driven by temperature inhomogeneities at the surface of the star, but rather by dust structures in the disk rotating at the Keplerian velocity.
}

overall shape of the distribution can be described as a gamma or a Weibull distribution ${ }^{10}$ (Weibull 1951), with a mean of $5.2 \pm 0.6 \mathrm{~d}$ and a variance of $13 \pm 3 \mathrm{~d}$. These density distributions have an asymmetric shape, with a concave, rapidly rising profile between zero and the peak of the distribution, and a convex, gradually decreasing profile afterwards. Regarding the two peaks observed in Fig. 5, the first occurs around $P \sim 1-2$ days, the second at $P \sim 3-4$ days. This is followed by a gradually decreasing tail of longer periods. When reducing the bin size from $1 \mathrm{~d}$ to $0.5 \mathrm{~d}$ to better resolve the structure of the peaks, these appear to be centered around $P \sim 1.3 \mathrm{~d}(1-1.5 \mathrm{~d})$ and $P \sim 3.3 \mathrm{~d}(3-3.5 \mathrm{~d})$ respectively; the computed $\pm \sigma$ width for each of the peaks is $0.8 \mathrm{~d}$. These numbers imply that the two peaks would no longer be resolved, but start to merge, if we adopted a bigger bin size (e.g., $1.5 \mathrm{~d}$ ). Similarly, if we retained a bin size of $1 \mathrm{~d}$ but shifted the bin centers along the $x$-axis in Fig. 5 , the global shape and properties of the histogram would be maintained for shifts of 0.1 or $0.2 \mathrm{~d}$, but shifts of 0.3 or $0.4 \mathrm{~d}$ would determine the two peaks in Fig. 5 to "disappear" as they redistribute into two neighboring channels. This is illustrated in Fig. B.1.

Attridge \& Herbst (1992) were the first to report evidence of a similar feature (two peaks) in the period distribution of a very young stellar cluster (the 2 Myr-old ONC). To explain this observational feature, the authors suggested that, contrary to the rapid rotators, the slow rotators may be experiencing magnetic braking through interaction with their circumstellar disk. This suggestion was later confirmed by Edwards et al. (1993). The bimodal period distribution for the $\mathrm{ONC}$, as defined by Attridge \& Herbst (1992), was strengthened in the follow-up study of Choi \& Herbst (1996), and its nature was later explored by Herbst et al. $(2001,2002)$ with special reference to stellar

10 The curve fitting was performed using the fitdistrplus package built for the $R$ environment, and employing the maximum likelihood estimation technique to assess which probability distribution provides the best fit to the observed distribution of values. 


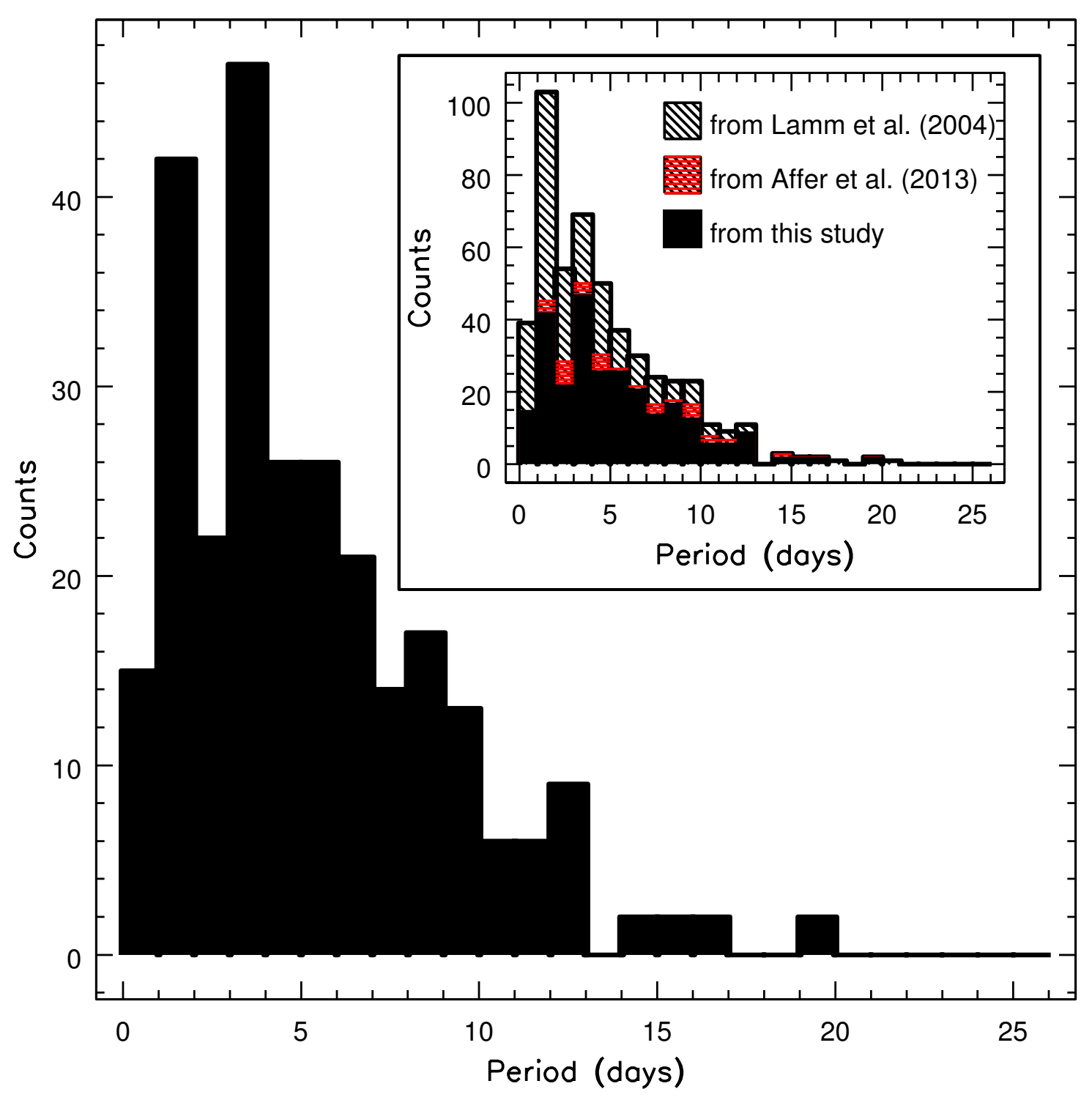

Fig. 5. Period distribution for the NGC 2264 cluster, as inferred from the population investigated in this study (black filled bars). Only single-period objects are considered here to build the histogram. The inset box to the right of the main period histogram shows the period distribution that would be derived, for the cluster, when completing our sample with the period detections obtained, in order of preference, by Affer et al. (2013, shaded in red) or Lamm et al. (2004, cross-hatched in black) for additional cluster members with no light curves in the CoRoT 2011 dataset.

mass. To assess a possible bimodal nature of the period distribution we obtain here for NGC 2264, we computed several statistical parameters, such as the kurtosis of the distribution (e.g., DeCarlo 1997) and the bimodality coefficient BC, which is in turn based on the kurtosis and skewness of the distribution (SAS Institute Inc. 2012). However, the high degree of asymmetry of the distribution and the presence of a heavy tail of slow rotators strongly affect the numerical values of these parameters, thus rendering the test inconclusive. Similarly, we attempted to apply the dip test statistics formulated in Hartigan \& Hartigan (1985) and Hartigan (1985) ${ }^{11}$, which consists in computing the unimodal distribution that best approximates the empirical cumulative distribution function observed in our sample and measuring the maximum difference between this fitting unimodal distribution and the empirical distribution. Again, the results of the test do not allow us to formally reject the null hypothesis that

\footnotetext{
${ }^{11}$ We used here the version of the test implemented in the diptest package for the $R$ environment.
}

the observed distribution is unimodal, with a $p$-value of 0.26 . Nevertheless, the presence of two peaks appears to be sound in the empirical period distribution for the cluster in Fig. 5; we will therefore abstain from calling this distribution bimodal, but retain the observational evidence of two peaks, and examine its possible implications in the context of young stars and rotational evolution in the following.

The inset panel in Fig. 5 shows a more populated period distribution for the cluster that would be obtained if we integrate our sample (reported in Table 4) with the periods derived for additional cluster members by, in order of preference, Affer et al. (2013) or Lamm et al. (2004) $)^{12}$. To complete the sample, we cross-correlated the full list of confirmed members from the CSI 2264 campaign (Cody et al. 2014) with the lists of periodic sources provided by Affer et al. (2013) and Lamm et al. (2004),

\footnotetext{
${ }^{12}$ No additional measurements from Makidon et al. (2004) are reported here because they amount to only a few sources, hence with no significant impact on the period distribution shown in the inset panel of Fig. 5.
} 
and selected common objects with no CoRoT light curve from the 2011 dataset, hence not included in the period analysis performed in this study. As can be observed, the addition of a few tens of objects (35) from the Affer et al.'s (2013) sample does not significantly modify the overall shape of the period distribution obtained from the present study. A far larger sample of additional periodic sources (190) can be retrieved from Lamm et al. (2004); about $75 \%$ of them have $R$-band magnitude fainter than 17 (limiting magnitude for the CoRoT sample, as mentioned in Sect. 2). When adding measurements from Lamm et al. (2004) to the period distribution obtained here, the first peak $(P=1-2 \mathrm{~d})$ is amplified relative to the second $(P=3-4 \mathrm{~d})$. However, this may be affected by a non-negligible fraction of aliases among the additional $\sim 1 \mathrm{~d}$ rotators detected by Lamm et al. (2004) in their ground-based campaign; indeed, about $10 \%$ of the objects common to this study and to Lamm et al.'s study, with assigned period between 1 and 3 days in the latter, are found to be slower rotators from the analysis of the CoRoT light curves (as illustrated in the right panel of Fig. 4).

\subsection{Period distribution: CTTS vs. WTTS}

We will now focus on the periods derived in this study for the sample of NGC 2264 members included in the CoRoT 2011 campaign. To investigate further the nature of the period distribution shown in Fig. 5 (main panel), we follow the member classification proposed in Venuti et al. (2014) and explore the rotation properties of cluster members with active accretion disks (CTTS) and of those without evidence of ongoing accretion and circumstellar material (WTTS) separately. The comparison between the period histograms obtained for the two classes is illustrated in Fig. 6 (left panel).

A visual inspection of the two distributions suggests that CTTS exhibit distinct rotation properties from WTTS. WTTS' distribution peaks at 1-2 d, and steadily decreases toward longer periods, with hints of a second, less prominent peak between 3 and $4 \mathrm{~d}$. Conversely, very few CTTS are found to exhibit rotation periods shorter than $2.5 \mathrm{~d}$; their distribution in periods exhibits a single peak around $P \sim 3-4 \mathrm{~d}$, and then decreases toward longer periods. A Kolmogorov-Smirnov (K-S) test (Press et al. 1992) applied to the two populations supports this idea of statistically distinct rotation properties for the two groups. Indeed, the test yields a probability of only $4 \times 10^{-3}$ that the two distributions in period corresponding to CTTS and WTTS are extracted from the same parent distribution. This is also consistent with the conclusions presented in previous studies of rotation in NGC 2264 (e.g., Cieza \& Baliber 2007; Affer et al. 2013).

If we compare the left panel of Fig. 6 with the overall period distribution of NGC 2264 shown in Fig. 5, the following inferences can be drawn: (i) the peak at short periods (1-2 d) observed in the global distribution is clearly associated with WTTS; (ii) the second peak observed in Fig. 5 ( $P=3-4 \mathrm{~d})$ takes contribution from both CTTS and WTTS. These results provide a clear indication of a statistical connection between disk and rotation properties across the cluster. The vast majority of fast rotators found among cluster members are objects which lack disk signatures. Figures 5 and 6 clearly illustrate that the first peak in the overall period distribution is associated with diskfree objects, and contains about $21 \%$ of WTTS with detected rotation period. Conversely, the second peak in Fig. 5 contains about $15 \%$ of the periodic WTTS and $21 \%$ of the periodic CTTS in our sample (6\% and $11 \%$, respectively, if we count objects in the peak only above the underlying "continuum"). This is also illustrated in the cumulative distributions in period relevant to the total, CTTS, and WTTS samples, shown in Fig. 7.

\subsection{Period distribution as a function of stellar mass}

In their review on the rotation properties and evolution of low-mass stars, Herbst et al. (2007) discuss a possible massdependence of the measured period distributions for young stars. Observational results in favor of this point were reported by Herbst et al. (2001) for the ONC ( 2 Myr) and by Lamm et al. (2004, 2005) in NGC 2264. In both cases, although quantitative differences exist between the rotation properties derived for the two clusters, a bimodal period distribution was derived by the authors for cluster members more massive than $\sim 0.4 M_{\odot}$, whereas lower-mass objects appear to spin faster on the average and define a possibly unimodal period distribution (see Fig. 2 of Herbst et al. 2007).

To test a possible mass-dependence in our data, we divided our sample into three similarly populated mass ${ }^{13}$ subgroups: (i) $M_{\star}<0.4 M_{\odot}$ (72 objects); (ii) $0.4 M_{\odot} \leq M_{\star} \leq 1 M_{\odot}$ (95 objects); (iii) $M_{\star}>1 M_{\odot}$ (86 objects). The separate period distributions for these three mass groups are shown in the right panel of Fig. 6. A simple visual inspection of these histograms would suggest that the measured period distribution does evolve, to a certain extent, as a function of mass: a peak of fast rotators $(P=1-2 \mathrm{~d})$ dominates the distribution in the lowest-mass group, albeit with a significant dispersion of objects at slower rotation rates; as stellar mass increases, this feature becomes less important compared to an emerging peak at slower rotation rates $(P=3-4 \mathrm{~d})$. However, no statistical support to this inference arises from the application of a two-sample KolmogorovSmirnov (K-S) test, applied to the lowest-mass and highest-mass groups (a $p$-value of 0.3 is obtained from the test, which does not allow us to discard the null hypothesis that the two populations are extracted from the same parent distribution $)^{14}$. This result does not change when considering CTTS and WTTS separately: both groups of objects exhibit similar mass properties, as ascertained by comparing their respective cumulative distributions in mass; no statistically supported evidence of a mass dependence in the rotation properties is inferred in either case ${ }^{15}$.

A key physical quantity to investigate the rotational evolution of stars is the specific angular momentum $j_{\text {star }}$ (see, e.g., Herbst \& Mundt 2005). This is linked to the period $P$ and radius $R_{\star}$ of the star according to the relation

$j_{\mathrm{star}}=k^{2} R_{\star}^{2} \omega=\frac{2 \pi k^{2} R_{\star}^{2}}{P}$,

where $k^{2}$ is the radius of gyration in units of stellar radius. In Fig. 8, we show the values of $j_{\text {star }}$ computed for objects in our sample as a function of mass. In the computation we assumed a

\footnotetext{
${ }^{13}$ Mass estimates are from Venuti et al. (2014).

${ }^{14}$ We adopted here the version of the test implemented in the stats package for the $R$ environment, and assuming as alternative hypothesis that the cumulative distribution function of the first population (the lower-mass group) lies above that of the second population (the highermass group).

${ }^{15}$ If we repeated the analysis on a possible mass dependence after integrating our sample with additional periodic sources from Lamm et al. (2004), which would mostly fall in the $M_{\star}<0.4 M_{\odot}$ bin, the null hypothesis that objects below $0.4 M_{\odot}$ and objects above $1 M_{\odot}$ share the same rotation properties would be rejected to the $5 \%$ level. However, this result may be affected by aliases at $P \sim 1 \mathrm{~d}$ detected from the ground, which would artificially increase the strength of the peak of fast rotators.
} 

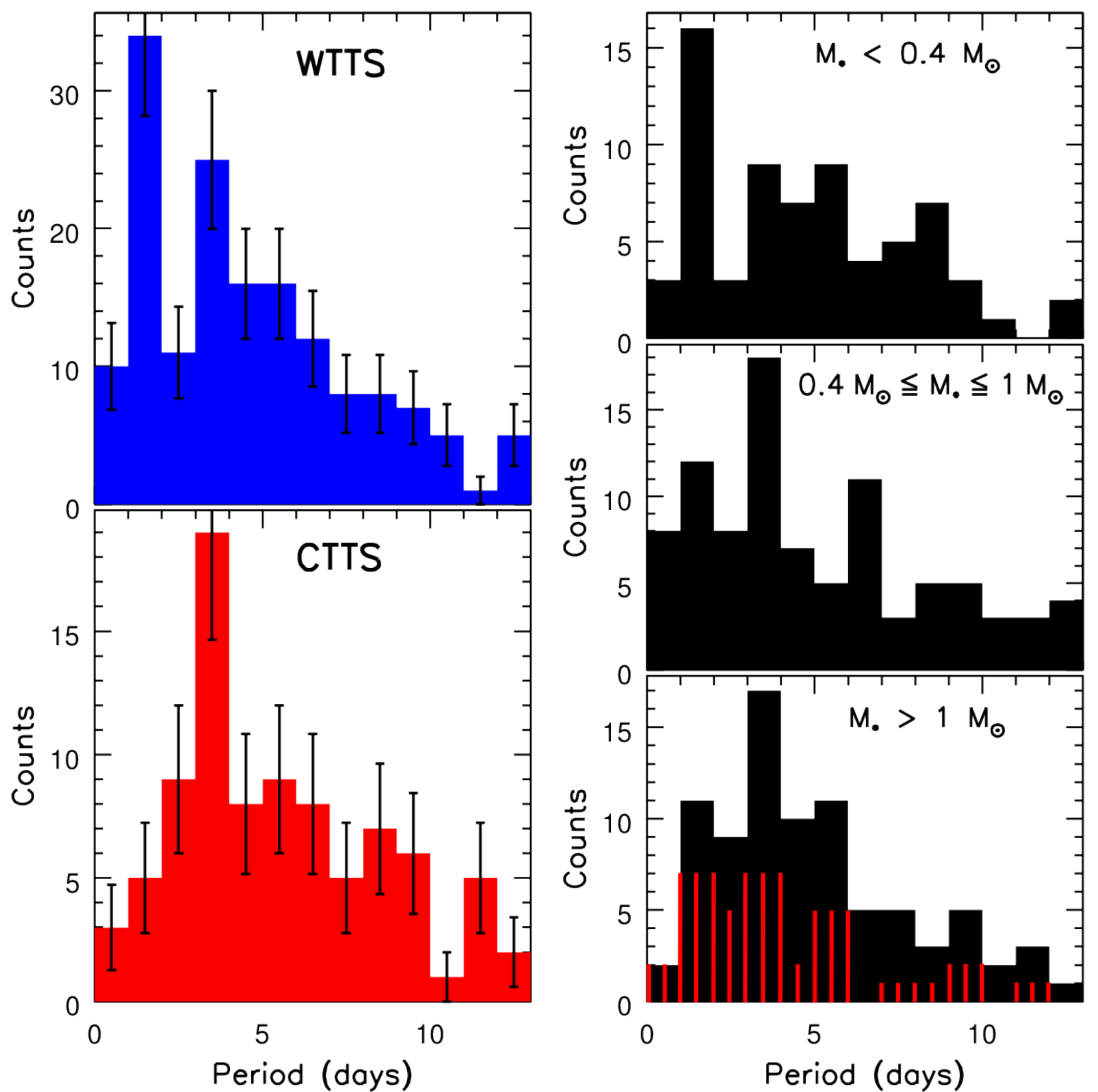

Fig. 6. Left panel: period distributions inferred separately for the populations of disk-free young stars (WTTS; blue) and disk-bearing objects (CTTS; red) in NGC 2264, probed in this study. Only periods comprised between $0 \mathrm{~d}$ and $13 \mathrm{~d}$ are shown here; in addition, only objects with single periodicity and with an associated WTTS/CTTS flag in Venuti et al. (2014) are considered to build the histogram. The error bars trace the Poissonian uncertainty computed on each histogram bin. Right panel: period distribution of NGC 2264 members as a function of stellar mass. The sample investigated for periodicity in this study is separated into three different mass bins: $M_{\star}<0.4 M_{\odot}$ (upper panel), $M_{\star}$ comprised between $0.4 M_{\odot}$ and $1 M_{\odot}$ (middle panel), $M_{\star}>1 M_{\odot}$ (lower panel). In the latter, the period distribution of objects with $M_{\star}>1.4 M_{\odot}$ is highlighted further as a superimposed histogram hatched in red.

constant value of 0.203 for $k^{2}$, following Vasconcelos \& Bouvier (2015) and their Fig. 12 for a cluster aged of $\sim 3$ Myr. Herbst et al. (2001) investigated the $j_{\text {star }}$ distribution of objects in the ONC, and found that $j_{\text {star }}$ is roughly independent of stellar mass over the mass range $0.1-1 M_{\odot}$. Since lower-mass stars are smaller (i.e., have smaller radii) than higher-mass stars, this result suggests that they tend to rotate faster. The $j_{\text {star }}$ vs. $M_{\star}$ distribution we derive here for NGC 2264 is consistent with this picture: no correlation is observed between the two quantities, but the same range of $j_{\text {star }}$ values is spanned at any given mass. This would support the visual inference from Fig. 6 of some mass dependence in the rotation properties of NGC 2264 members, with lower-mass objects exhibiting on average shorter rotation periods than higher-mass objects.
The third mass group $\left(M_{\star}>1 M_{\odot}\right)$ includes the critical mass $\left(M_{\star} \sim 1.3 M_{\odot}\right)$ at which a break in rotation properties is observed among solar-type stars (Kraft 1967). Objects more massive than this threshold have largely radiative interiors, and spend little time along the convective track during their PMS evolution. Convection plays an important role in braking the stars, by powering stellar winds that carry away angular momentum. Massive stars, which are deprived of this mechanism, experience a different rotational evolution from less massive stars with deep convective envelopes, and reach the ZAMS with rotational velocities nearly an order of magnitude higher than the latter. To check whether this effect is already seen at the age of NGC 2264, we selected objects below and above $M_{\star}=1.4 M_{\odot}$ in this mass group, and compared their respective frequencies in the $P<6 \mathrm{~d}$ region 

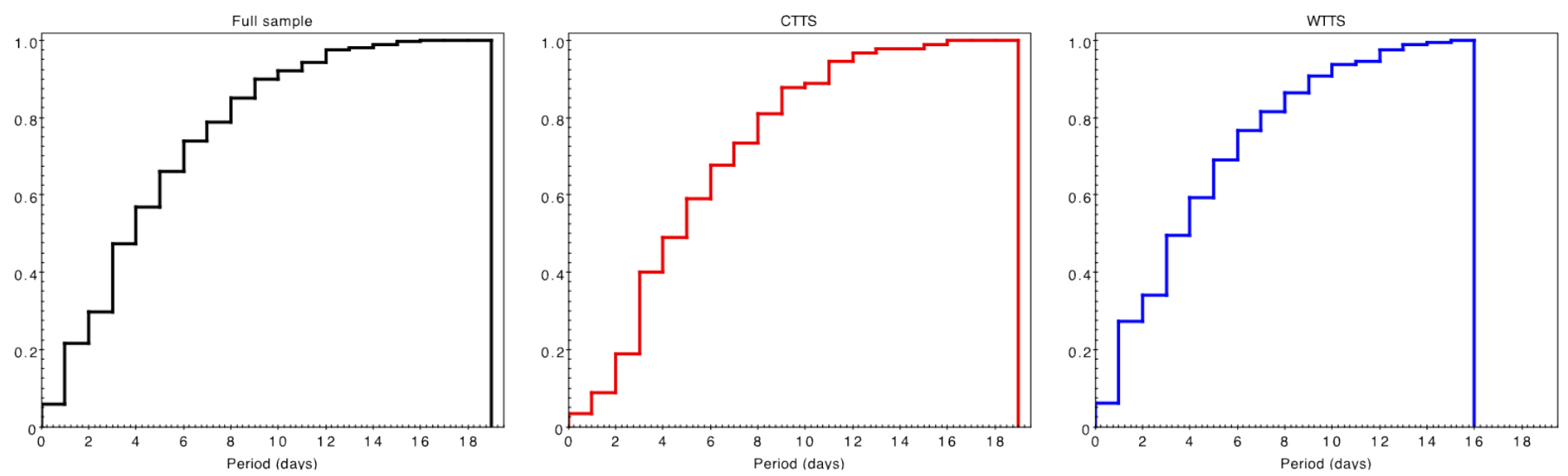

Fig. 7. Normalized cumulative distributions in period associated with the full sample of NGC 2264 members (left panel), the population of CTTS (middle panel), and the population of WTTS (right panel) analyzed in this study. Two prominent jumps of similar extent (0.16 and 0.17$)$ appear in the cumulative distribution for the full sample at $P=1 \mathrm{~d}$ and $P=3 \mathrm{~d}$, respectively. In the case of the CTTS, only one prominent jump of 0.21 at $P=3 \mathrm{~d}$ is seen. In the case of the WTTS, two prominent discontinuities can again be observed, one steeper at $P=1 \mathrm{~d}(0.21)$ and one smaller at $P=3 \mathrm{~d}(0.15)$.

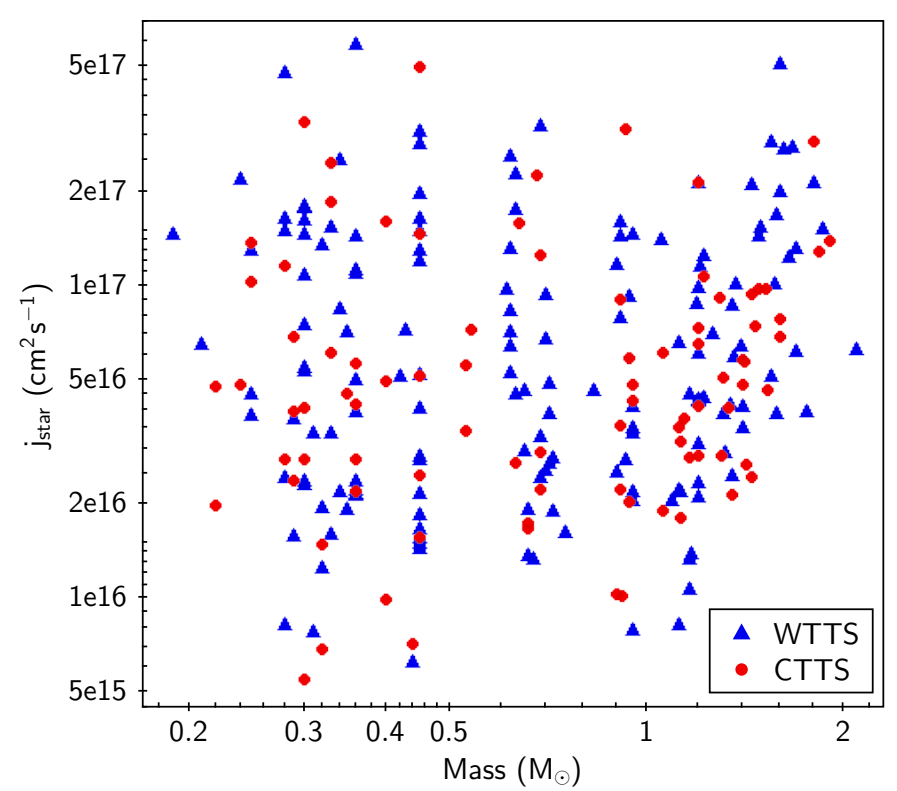

Fig. 8. Specific angular momentum, as a function of stellar mass, measured for CTTS (red circles) and WTTS (blue triangles) in our sample. Hints of a break in rotation properties can be observed around $M_{\star} \sim 1.4 M_{\odot}$, as discussed in the text.

of the histogram shown in the bottom right panel of Fig. 6. This period range contains $60 \%$ of objects with $1 M_{\odot}<M_{\star} \leq 1.4 M_{\odot}$ and $85 \%$ of objects with $M_{\star}>1.4 M_{\odot}$ (highlighted in red on the period distribution shown in the bottom right panel of Fig. 6). Moreover, about $70 \%$ of objects in this mass group with $P<2 \mathrm{~d}$ have $M_{\star}>1.4 M_{\odot}$. The median period measured across objects with mass between 1 and $1.4 M_{\odot}$ is $5 \mathrm{~d}$, while the median period measured for objects with mass above $1.4 M_{\odot}$ is $3 \mathrm{~d}$. This comparison suggests that some separation in rotation properties below and above $M_{\star} \sim 1.4 M_{\odot}$ might already be present at an age of a few Myr, although with no clear break, but a substantial overlap in period distributions. To test whether objects more massive than $1.4 M_{\odot}$ may blur the mass trend in rotation properties, we repeated the K-S test between the lowest-mass and highest-mass groups in Fig. 6 after removing objects with $M_{\star} \geq 1.4 M_{\odot}$. This returned a $p$-value of 0.09 , lower than that obtained when applying the test to the complete $M_{\star}<0.4 M_{\odot}$ and $M_{\star}>1.0 M_{\odot}$ groups. Thus, the null hypothesis that the period distributions associated with the two mass groups are extracted from the same parent distribution is rejected at the $10 \%$ significance level, but not at the $5 \%$ significance level.

\subsection{Period distribution as a function of variability class}

Figure 9 illustrates the period distribution for NGC 2264 members which fall respectively into the strictly periodic (spotted), quasi-periodic (spotted), dipper, burster and stochastic light curve classes, defined based on the morphology of the CoRoT light curves (Appendix A). The first two classes include both WTTS and CTTS, whereas the classes of dippers, bursters and stochastic light curves are specific to disk-bearing objects.

Among sources with spot-dominated light curves, a larger fraction is recovered in the quasi-periodic class than in the strictly periodic class (104 vs. 75). In addition, the CTTS/WTTS ratio differs significantly between the two classes: less than $10 \%$ of strictly periodic sources are CTTS, while nearly $30 \%$ of spotted sources with quasi-periodic light curves are CTTS. This can be understood if we consider that the light curves of CTTS typically result from a variety of co-existing mechanisms (spot modulation, spot evolution, accretion); therefore, their morphology may exhibit rapid changes, even when the periodicity does not evolve during the monitored time. Conversely, the light variations of WTTS are driven by cold spot modulation; these spots are often stable and long-lived, thus leading to stable light curve morphology on tens or hundreds of rotational cycles. Nevertheless, a significant fraction of WTTS falls into the definition of quasi-periodic light curves; this may reflect spot evolution or migration on timescales of a few stellar periods.

The different ratio of CTTS to WTTS in the two classes is also reflected in the properties of the relevant period histograms. The period distribution of purely periodic light-curve objects include more fast rotators than the group of quasi-periodic light curves, and conversely, more slow rotators are included in the quasi-periodic sample than in the strictly periodic sample. No fast rotators have light curves in the dipper, burster or stochastic categories; the periods measured among these latter samples span a broad range of values, from a few to several days. Only a few sources with periodic signatures are detected among the burster and the stochastic light curve types; this is due to the 


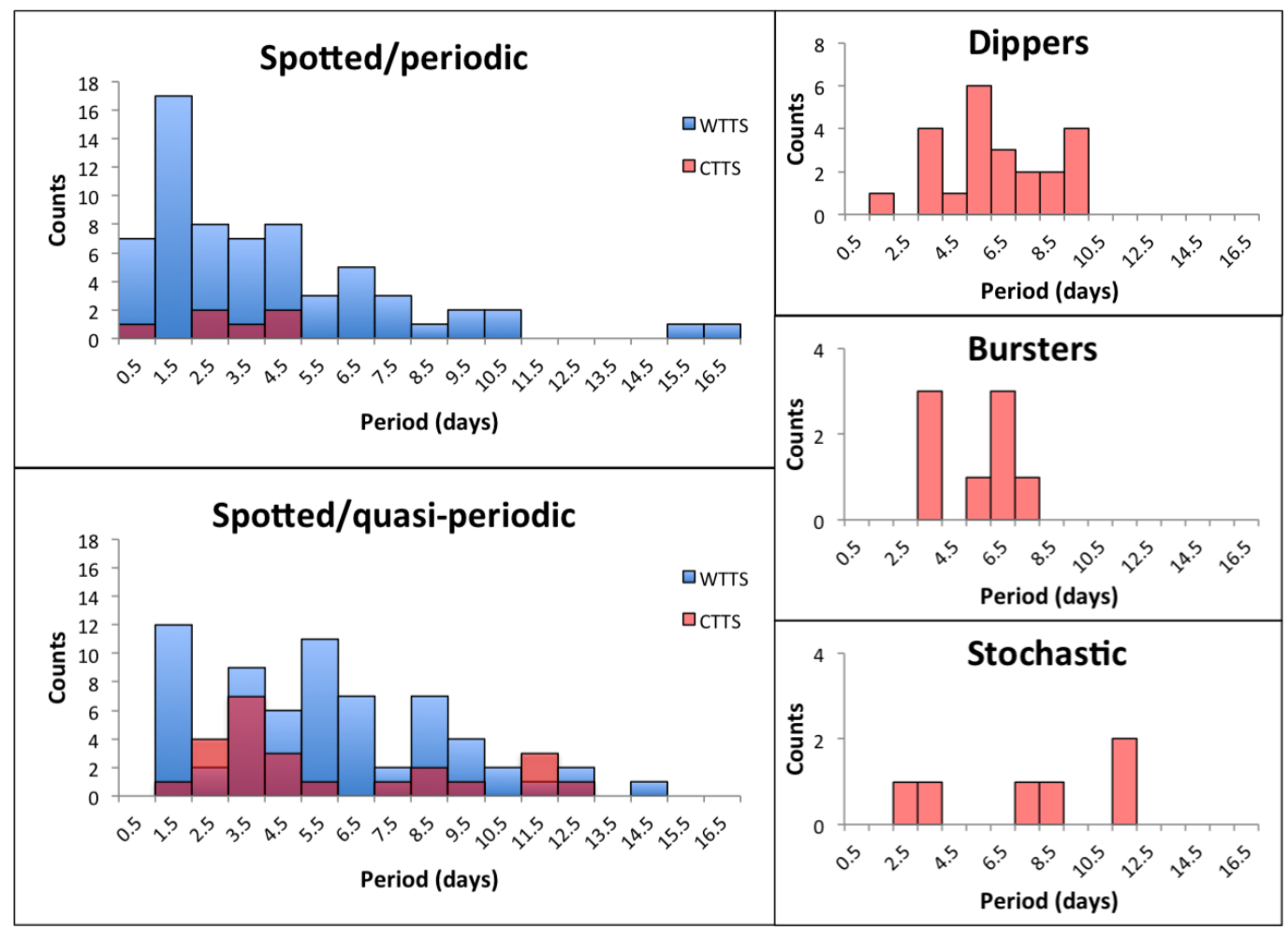

Fig. 9. Period distributions of different groups of NGC 2264 members, divided according to the morphology of their CoRoT light curve. WTTS are illustrated in blue, CTTS are overlapped in red.

episodic nature of the physical processes which dominate their light variations (Stauffer et al. 2014, 2016).

\subsection{Are CTTS periods similar in nature to WTTS periods?}

The derivation of stars' rotation periods from monitoring their photometric variability relies on the assumption that these light variations are dominated by localized temperature inhomogeneities at the stellar surface. These inhomogeneities would then modulate the apparent luminosity of the stars as these spin on their axes, with a characteristic timescale of variability equal to the rotation period of the star. Recently, Artemenko et al. (2012) questioned the validity of this assumption as a general rule for CTTS. The authors examined the light curve of about 50 CTTS in the photometric catalog of Grankin et al. (2007) to derive their rotational periods via power spectrum analysis. The rotational period of a star can be expressed as

$P=\frac{2 \pi R_{\star}}{v} \equiv \frac{2 \pi R_{\star} \sin i}{v \sin i}$

where $v$ is the equatorial velocity, $i$ is the inclination ${ }^{16}$ of the system, and $v \sin i$ is the projected rotational velocity, measured from spectroscopic observations. An estimate of $\sin i$ can then be derived from Eq. (8), if $P, v \sin i$ and $R_{\star}$ are known. If the value of period $P$ we measure is photospheric, then estimates of $\sin i \lesssim 1$ ought to be derived from Eq. (8). Instead, $\sin i$ estimates larger than 1 were obtained by Artemenko et al. (2012) across their sample of CTTS. Objects were found to trace a unique sequence on the $\sin i$ vs. $P$ diagram, with $\sin i$ tending to increase with $P$ (see their Fig. 2). The authors suggested that in some cases, the measured photometric periods for CTTS do not arise from surface spot modulation, but from clumps of dust in the

${ }_{16}$ Angle between the rotation axis and the line of sight to the observer. disk which periodically occult the stellar photosphere, at a rate corresponding to the Keplerian orbit where they are located in the disk. In this case, the formal application of Eq. (8) to derive $\sin i$ will yield values larger than 1 , since the measured period originates at distances larger than the stellar radius (unless they arise from the co-rotation radius, or close to it).

Conversely, there should be no ambiguity on the nature of the photometric periods measured for disk-free young stars; therefore, the same test, applied to a sample of WTTS, is expected to produce estimates of $\sin i$ systematically lower than 1, conditional upon the accuracy of the stellar parameters determined for those objects.

Here we follow Artemenko et al.'s (2012) approach to test the nature of the photometric periods we measured for NGC 2264 members, and invert Eq. (8) to derive sin $i$ estimates for CTTS and WTTS in our sample. Values of $\sin i$ are calculated as

$\sin i=0.0195 P(v \sin i) / R_{\star}$,

where $P$ is expressed in days, $v \sin i$ is in $\mathrm{km} \mathrm{s}^{-1}$, and $R_{\star}$ in solar radii $R_{\odot}$. We use $R_{\star}$ estimates from Venuti et al. (2014), while $v \sin i$ values are retrieved from the study of Baxter et al. (2009). For this test, we selected 51 CTTS and 81 WTTS, common to the samples of Venuti et al. (2014) and Baxter et al. (2009), with single periodicity detected in the present study. The results of this computation are shown, as a function of period, in Fig. 10.

When comparing the $v \sin i$ distributions for CTTS and WTTS in our sample, no statistically significant difference between the two is found, although the average $v \sin i$ is larger in the WTTS group than in the CTTS group. A similar analysis was presented by Rhode et al. (2001) for the PMS population of the ONC. In the majority of cases (84\%) across our sample, values of $\sin i \leq 1$ are obtained, within the associated uncertainties, from Eq. (9). This result is similar to that obtained by 

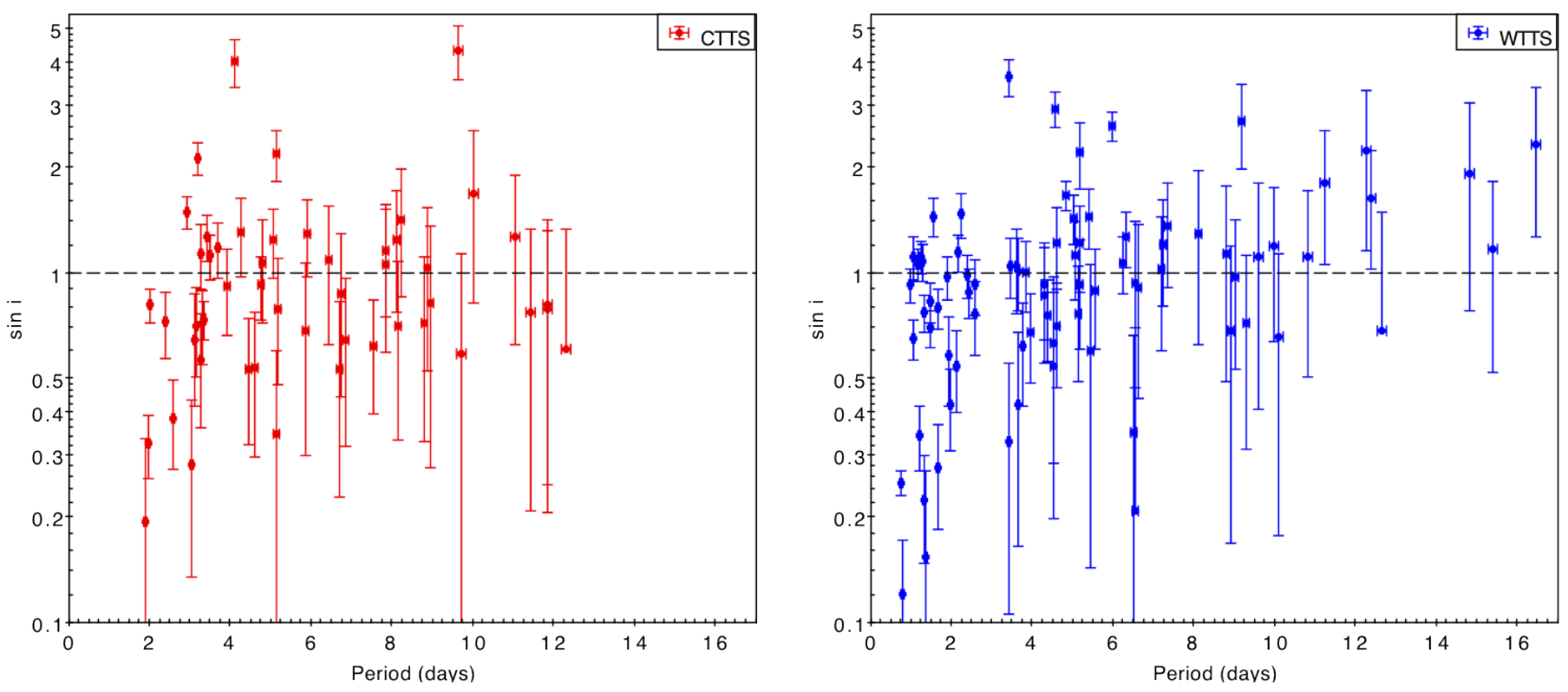

Fig. 10. Estimates of sin i obtained, as a function of the rotation period, for CTTS (red; left panel) and WTTS (blue; right panel) in our sample with $v$ sin $i$ measurements from Baxter et al. (2009), periods from this study and $R_{\star}$ estimates from Venuti et al. (2014). The sin $i=1$ level is marked as a black dashed line. Horizontal error bars are derived following Eq. (6). Vertical error bars are derived by standard error propagation on Eq. (9). A typical uncertainty of $5 \mathrm{~km} \mathrm{~s}^{-1}$ is assumed on $v \sin i$; this corresponds to the median difference between $v \sin i$ estimates by Baxter et al. (2009) and those derived by S. Alencar from CSI 2264 VLT/FLAMES observations, for objects common to the two samples. Similarly, a typical uncertainty of $0.2 R_{\odot}$ is adopted for $R_{\star}$, that is the median difference between $R_{\star}$ estimates obtained by Venuti et al. (2014) and those of Rebull et al. (2002), for objects in common.

Artemenko et al. (2012). As noted by the latter, only a few points appear at values of $\sin i<0.2$; this is a selection effect due to the fact that for low inclinations (nearly pole-on objects), the photometric modulation is difficult to detect. A number of objects fall above the $\sin i=1$ line in Fig. 10; interestingly, a fraction of both the CTTS $(6 / 51$, or $11.8 \%)$ and the WTTS $(15 / 81$, or $18.5 \%$ ) groups are found in this region of the diagram. For both CTTS and WTTS, the average sin $i$ computed neglecting sources with $\sin i$ estimates larger than 1 is $\langle\sin i\rangle=0.6 \pm 0.2$; this value is consistent with that found by Rhode et al. (2001) for the ONC. In at least a few of the 6 CTTS with $\sin i$ estimate larger than 1 , this result may be severely affected by an erroneous $v \sin i$ measurement: largely discrepant (lower) $v \sin i$ values from Baxter et al.'s (2009) estimates are derived, for the same sources, from VLT/FLAMES spectra obtained within the CSI 2264 campaign. Among the 15 WTTS with $\sin i>1$ in Fig. 10, an assessment of the impact of uncertainties on $v \sin i$ measurements is more complicated, as not many of them have additional $v \sin i$ derivations for comparison purposes. In the few cases where such a comparison is possible, the $v \sin i$ measurements from different sources are not too dissimilar from each other, and the estimate of $\sin i$ obtained is only marginally larger than 1; the discrepancy here can then likely be explained in terms of uncertainties on the parameters adopted for the sources. While in a few cases it may be possible that these objects with no sign of accretion might still possess some material in the circumstellar environment, most of these objects exhibit strong evidence of being disk-free young cluster members. Therefore, uncertainties on the nominal parameters used for the $\sin i$ computation are likely to affect significantly the results shown in Fig. 10. At any rate, the conclusion we may derive from this figure is that, for the majority of the objects investigated here, the period measured from the CoRoT light curves is likely photospheric, and hence corresponds to the spin rate of the star. Thus, the distribution in $\sin i$ appears to be independent of the accretion status of the objects.

\section{The rotation - accretion connection in NGC 2264}

\subsection{Rotation properties vs. disk accretion indicators}

Figure 6 illustrates that statistically distinct, though overlapping, behaviors in rotation properties characterize cluster members with accretion disk signatures with respect to disk-free sources. Namely, a pronounced peak of fast rotators $(P \sim 1-2 \mathrm{~d})$ appears in the period distribution pertaining to WTTS, whereas very few fast rotators are found among objects still surrounded by disks.

As discussed in Sect. 1, the impact of accretion disks on the early rotational evolution of young stars is an issue that has long been debated over the past decades. Observational evidence has been gathered in support of a positive connection between the presence of disks and the measured rotation rates within coeval populations of young stars; objects still surrounded by disks, and hence presumably in magnetospheric interaction with them, appear to rotate more slowly, on the average, than young stars whose disks have already disappeared. A number of studies (e.g., Herbst et al. 2002; Rebull et al. 2006; Cieza \& Baliber 2007) have shown that the frequency of objects with near-infrared excess (indicative of dusty inner disks) increases with the rotation period, hence lending credit to this "disk-locking" scenario. Moreover, recent studies using Monte Carlo simulations to investigate the early rotational evolution of low-mass stars (e.g., Vasconcelos \& Bouvier 2015) have shown that, starting from the disk-locking assumption, the period distributions observed for young clusters of different ages can be reproduced reasonably well. However, as illustrated in Sect. 1 (see also Bouvier et al. 2014), no definite consensus has yet been reached on this issue, and which mechanisms provide an effective source of braking in young stars is still a matter of controversy. 


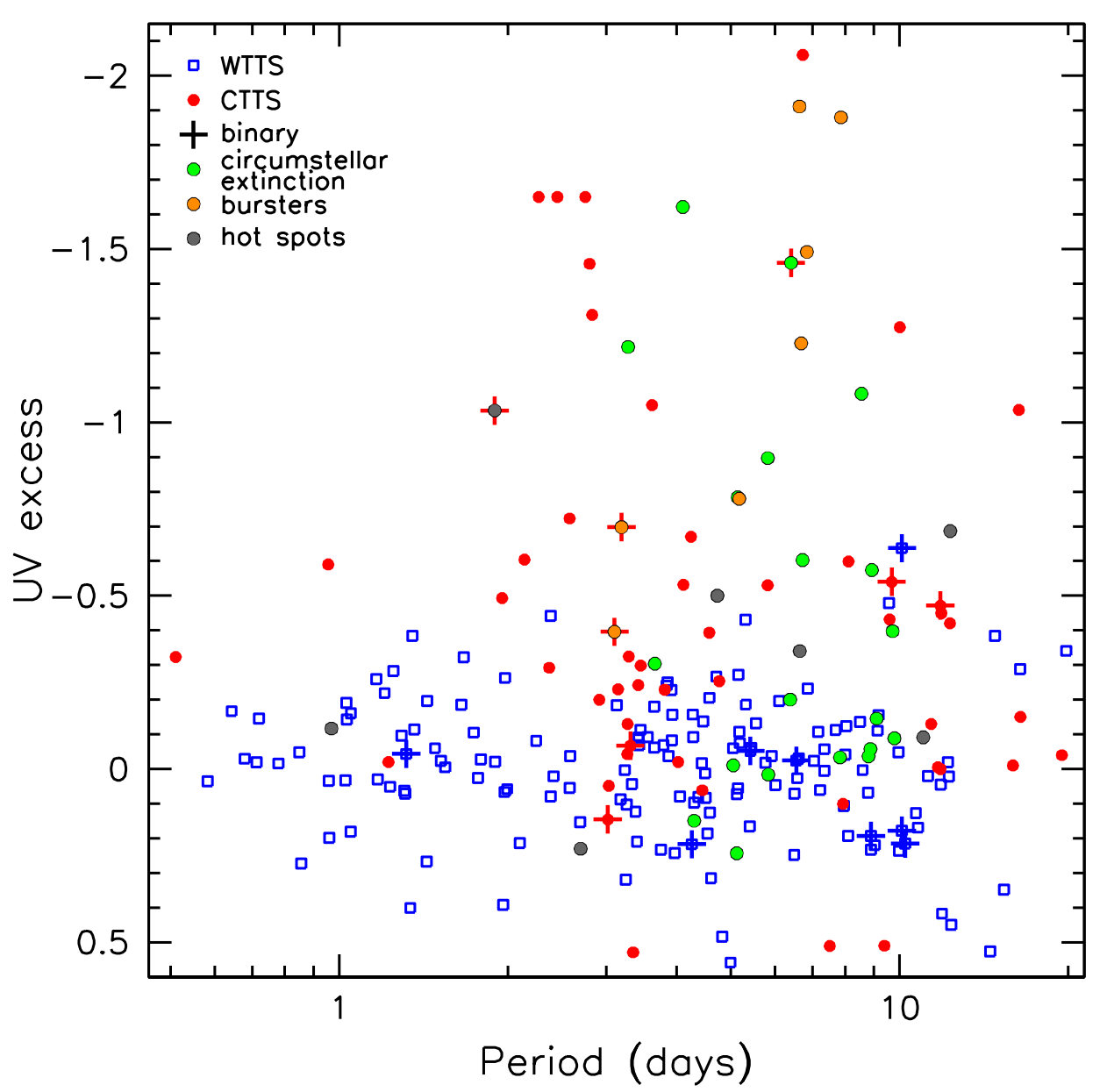

Fig. 11. UV excesses measured for NGC 2264 cluster members as described in Venuti et al. (2014) are compared to their measured rotation periods, reported in Table 4 of the present study. Filled dots correspond to disk-bearing objects (CTTS), while disk-free WTTS are indicated as empty blue squares. The different color groups among CTTS correspond to different variable classes, as detailed in the legend (green indicates light curves dominated by flux dips; orange corresponds to stochastic bursters; grey is used for CTTS with light curves dominated by spot modulation; red corresponds to other CTTS members whose light curve is not ascribed to any specific class among the ones listed before). A cross superimposed on a dot or square signifies that the corresponding object is a known spectroscopic binary.

The magnetospheric accretion process plays an important role in regulating the star-disk interaction during the first few Myr of a stellar lifetime. Measurements of the infrared excess of YSOs, although providing diagnostics of the presence of dusty disks around these young stars, are not able to probe the rate of mass accretion onto the central source. UV excess measurements are, instead, a direct indicator of accretion, as they probe the hot excess emission which arises from the accretion shock at the stellar surface. First studies to investigate a possible correlation between UV excesses and rotation periods include Rebull (2001) in Orion and Makidon et al. (2004) in NGC 2264, although no conclusive evidence could be drawn from those analyses. Later, Fallscheer \& Herbst (2006) determined UV excess estimates for a sample of 95 NGC 2264 members with known rotation periods from the studies of Makidon et al. (2004) or Lamm et al. (2004), and compared these two quantities to show the presence of an overall association between the two: slowly rotating stars appeared to be more likely to have large UV excesses (and hence, strong ongoing accretion activity) than faster rotators.

In Fig. 11, we report the same comparison for the broader sample available for NGC 2264 from the CSI 2264 campaign. The sample of members and their classification as CTTS or WTTS, as well as their measured UV excesses, follow from the
CFHT-based study of Venuti et al. (2014) ${ }^{17}$; the rotation periods are those derived in the present study and reported in Table 4. WTTS distribute horizontally across the whole period range, and exhibit no UV excess within an uncertainty of \pm 0.2 mag. Conversely, very few CTTS are found at periods shorter than 1.52 days; furthermore, a dearth of strong accretors (UV excess larger, i.e., more negative, than $-0.75 \mathrm{mag}$ ) with short rotation periods is clear in the diagram. The features of Fig. 11 do not exhibit any mass dependence, as the same qualitative diagram is recovered when splitting the sample into the three mass bins indicated on the right panel of Fig. 6. These features recall the analogous diagram presented in Rebull et al. (2006, Fig. 3), which juxtaposes rotation periods and mid-IR excesses measured from Spitzer/IRAC data, in the case of Orion. There, the authors found that objects with clear disk signatures stand out with respect to those with no IR excess, and are clustered at periods longer than $\sim 1.8$ days; conversely, objects with no evidence of disk were found to span the whole range in periods (from $\sim 0.3$ to 10 days), as is the case here.

${ }^{17}$ See in particular Eq. (9) of that paper for details on how the UV excess measurements were obtained; see also discussion in Sect.3.2 of Venuti et al. (2015) regarding the significance of such UV excess measurements for WTTS. 
When exploring the distribution in $\dot{M}_{\text {acc }}{ }^{18}$ of CTTS as a function of period, no 1-to-1 correspondence between the two quantities is found; rather, at a given period, $\dot{M}_{\text {acc }}$ values can span over an order of magnitude. This variety of $\dot{M}_{\text {acc }}$ regimes at a given $P$ may reflect distinct accretion mechanisms (Romanova et al. 2004; Kulkarni \& Romanova 2008), or correspond to different accretion histories (Matt et al. 2012), perhaps linked to varying properties of the circumstellar environment for individual objects (e.g., disk masses; see Manara et al. 2016). The fact that a given rotation period includes objects with diverse accretion rates may indicate that distinct components of the stardisk environment are predominant in determining the two sets of properties: the accretion rate is ultimately regulated by the smallscale magnetic field structure in proximity of the stellar surface; conversely, the star-disk coupling and angular momentum transfer is dominated by the large-scale, ordered dipole component of the stellar magnetosphere (see discussion in Gregory et al. 2012). The fact that the magnetic field strength may vary from object to object was also suggested by Muzerolle et al. (2001) to explain the lack of an observed correlation between $P$ and $\dot{M}_{\text {acc }}$, which would be expected from a theoretical standpoint if higher accretion rates tend to push the disk truncation radius $R_{\mathrm{T}}$ closer to the star (cf. Sect. 5.2.1 and Eq. (10)).

\subsection{Disk-locking?}

As discussed in Sect. 1, one of the most debated issues regarding angular momentum regulation in young stars concerns the role played by star-disk interaction, and the possible magnetic star-disk locking that would prevent the objects from spinning up during the disk accretion phase. In this section, we explore some main concepts related to the magnetospheric accretion picture and test their agreement with the observational parameters measured for NGC 2264 disk-bearing objects.

\subsubsection{Truncation radius}

A critical parameter in the star-disk magnetospheric interaction is the location of the truncation radius $R_{\mathrm{T}}$, i.e., the radial distance from the star at which the inner disk is disrupted by the stellar magnetosphere. Another important distance in the picture of magnetospheric accretion is the corotation radius $R_{\mathrm{C}}$, that is, the radius of the disk annulus where the Keplerian velocity of the disk equals the angular velocity of the star. Disk material orbiting the star at distances $R<R_{\mathrm{C}}$ rotates faster than the star, while at radial distances $R>R_{\mathrm{C}}$ the magnetosphere threading the disk rotates faster than the corresponding Keplerian orbit in the disk. Thus, the outcome of the magnetosphere-disk interaction will depend on the mutual position of $R_{\mathrm{T}}$ and $R_{\mathrm{C}}$. When $R_{\mathrm{T}}<R_{\mathrm{C}}$, at the interaction interface a negative magnetic torque will be exerted on the disk material; this favors its channeling along the magnetic field lines and subsequent accretion onto the star. Conversely, when $R_{\mathrm{T}}>R_{\mathrm{C}}$, at the interaction interface the magnetosphere rotates faster than the disk material, which is then accelerated along the azimuthal direction and may eventually be expelled radially from the system (the so-called "propeller" regime; Ustyugova et al. 2006). In the latter scenario, no stable funnel-flow accretion can occur.

If the physical conditions for the creation of stable, magnetically driven accretion funnels are met, the $R_{\mathrm{T}}$-to- $R_{\mathrm{C}}$ ratio in a given system is expected, from a theoretical standpoint, to be

\footnotetext{
${ }^{18}$ Accretion rates are derived from UV excesses shown in Fig. 11 as described in Venuti et al. (2014).
}

related to the stellar mass $M_{\star}$, radius $R_{\star}$, accretion rate $\dot{M}_{\text {acc }}$, rotation period $P$ and magnetic field $B_{\star}{ }^{19}$ according to the following equation (Bessolaz et al. 2008):

$$
\frac{R_{\mathrm{T}}}{R_{\mathrm{C}}} \simeq 0.25 m_{\mathrm{S}}^{2 / 7} B_{\star}^{4 / 7} \dot{M}_{\mathrm{acc}}^{-2 / 7} M_{\star}^{-10 / 21} R_{\star}^{12 / 7} P^{-2 / 3},
$$

where $m_{\mathrm{S}}$ is the Mach number at the disk midplane, $B_{\star}$ is normalized to $140 \mathrm{G}, M_{\star}$ is normalized to $0.8 M_{\odot}, R_{\star}$ is normalized to $2 R_{\odot}, \dot{M}_{\text {acc }}$ is normalized to $10^{-8} M_{\odot} / \mathrm{yr}$ and $P$ is normalized to $8 \mathrm{~d}$. To test whether this prescription yields $R_{\mathrm{T}} / R_{\mathrm{C}}$ estimates consistent with the picture of stable funnel-flow accretion for NGC 2264 members, we selected a subsample of accreting objects for which all parameters listed in Eq. (10) are known from this study (rotation period) and from Venuti et al. (2014, stellar parameters and mass accretion rate). To each of these objects, a value of $B_{\star}$ was assigned, following Gregory et al. (2012), based on their estimated radiative core mass to stellar mass ratio $\left(M_{\text {core }} / M_{\star}\right)$, deduced from Siess et al.'s (2000) evolutionary tracks. Specifically, a value of $B_{\star}=1.5 \mathrm{kG}^{20}$ was adopted for objects with $M_{\text {core }} / M_{\star}=0$ (i.e., fully convective stars); $B_{\star}=0.6 \mathrm{kG}$ was used for objects with $0<M_{\text {core }} / M_{\star} \lesssim 0.4$; $B_{\star}=0.1 \mathrm{kG}$ was adopted for objects with a developed radiative core $\left(M_{\text {core }} / M_{\star} \gtrsim 0.4\right)$. Objects considered for this test are listed in Table 2 , where the relevant $M_{\star}, R_{\star}, \dot{M}_{\text {acc }}, M_{\text {core }} / M_{\star}$ and $P$ parameters are reported. Following Bessolaz et al. (2008), two values of $m_{\mathrm{S}}, 1$ and 0.5 , were considered for the computation of $R_{\mathrm{T}} / R_{\mathrm{C}}$; both sets of results are illustrated in Fig. 12 .

The results of the $R_{\mathrm{T}}$ and $R_{\mathrm{T}} / R_{\mathrm{C}}$ computation for this subset of objects are shown, as a function of $P$, in Fig. 12. As ensuing from Eq. (10), stronger dipolar fields tend to disrupt the disks at larger radii; this is reflected in the vertical separation of the three different color groups (corresponding to different $B_{\star}$ assumptions) on the diagrams. The right panel of Fig. 12 shows that typical $R_{\mathrm{T}}$ estimates range from a few to several stellar radii (see also Johnstone et al. 2014). Values of $R_{\mathrm{T}} / R_{\star}<1$ are obtained from Eq. (10) for the group of objects with the largest radiative cores; this may indicate that the average value of $B_{\star}$ adopted for these sources is an underestimate to the actual magnetic field strength. In the majority of cases, the ratio of the truncation-to-corotation radius, illustrated in the left panel of Fig. 12, is smaller than 1 or close to 1 ; this is consistent with the expected behavior in the magnetospheric accretion picture, as discussed earlier. At the same time, a $R_{\mathrm{T}} / R_{\mathrm{C}}$ ratio larger than 1 is obtained for a small group of objects in our sample, among the stars with $M_{\text {core }} / M_{\star}=0$. Although the derived values of $R_{\mathrm{T}}$ may be affected by uncertainties on the stellar parameters and especially on the magnetic field intensity, which is not constrained here on an individual basis, it is interesting that objects with estimated $R_{\mathrm{T}} / R_{\mathrm{C}}>1$ are clustered at short rotation periods

${ }^{19}$ Strength of the dipolar component of the stellar magnetic field at the equator. As discussed in Gregory et al. (2012), the magnetic field hosted by $\mathrm{T}$ Tauri stars can strongly depart from a pure dipole; the degree of complexity of the magnetic field at the stellar surface, and the importance of higher-order components relative to the dipolar component, tend to increase, along the HR diagram, from fully convective stars to objects with a radiative core. However, the dipolar component of the magnetic field dominates on the large scale, as it decays more slowly with distance from the central source; hence, it is the dipolar component that regulates the star-disk interaction at the truncation radius.

${ }^{20}$ This value quoted here, as the following ones, are average estimates of the polar dipole strength for objects in different $M_{\text {core }} / M_{\star}$ regimes, as discussed in Gregory et al. (2012). However, in Eq. (10), the magnetic field is that measured at the equator. For a dipole, this corresponds to half the strength measured at the magnetic pole. 
in Fig. 12 (left panel). High stellar spin rates push the corotation radius closer to the star, hence favoring a scenario where the disk is truncated beyond the corotation radius. As mentioned earlier, in this scenario the inner disk material may be ejected from the system along open field lines, in the propeller regime (Ustyugova et al. 2006), carrying away angular momentum. This mechanism can efficiently spin down the star on timescales of $\lesssim 10^{6}$ yr (Ustyugova et al. 2006), shorter than the typical CTTS lifetimes of several Myr. Therefore, this would suggest that objects accreting in the propeller regime at the age of NGC 2264 might be expected to lie on the longer-period side of Fig. 12, rather than on the short-period side. Objects with the largest $R_{\mathrm{T}} / R_{\mathrm{C}}$ exhibit small UV excesses (and, therefore, weak $\dot{M}_{\mathrm{acc}}$ ) with respect to the bulk of NGC 2264 disk-bearing members, as inferred when comparing the $R_{\mathrm{T}} / R_{\mathrm{C}}$ estimates derived here to the accretion parameters derived in Venuti et al. (2014) for the whole NGC 2264 sample. A few of them would be classified as significantly younger $\left(<10^{6} \mathrm{yr}\right)$ than the other cluster members following Siess et al.'s (2000) model tracks on the H-R diagram of the cluster. This might suggest that they are young objects, accreting in a propeller regime, which have not yet been significantly spun down by the star-disk interaction mechanism. Nevertheless, the global inference we may derive from Fig. 12 is that there is no relationship between the truncation-to-corotation radius ratio and the rotation period of disk-bearing sources in NGC 2264 , in the sense that values of $R_{\mathrm{T}} / R_{\mathrm{C}}$ consistent with the magnetospheric accretion picture are found across the whole period range.

Gregory et al. (2012) suggested that the magnetic topology and its evolution as the star ages may have a direct impact on the rotational evolution of young stars. Namely, as the dipole component becomes weaker and the field complexity increases when the stars start to develop a radiative core, the magnetic ram pressure close to the truncation radius will decrease and hence the disk may push closer to the star. At this stage, the star would start to spin up due to the combined effects of the magnetosphereinner disk angular momentum exchange, of the accretion process onto the star, and of the stellar contraction. Therefore, a connection would be expected between the measured rotation period of the star and the strength/complexity of its magnetic field. Johnstone et al. (2014) investigated this connection across a sample of 10 CTTS with reconstructed magnetic maps from the MaPP project (e.g., Donati et al. 2010), and found that sources which host a stronger dipolar field tend to be associated with longer rotation periods. Even though no detailed knowledge of the magnetic topology is available for NGC 2264 members investigated here, some indications on a possible connection between stellar rotation and inner structure may be inferred by comparing how objects belonging to different $M_{\text {core }} / M_{\star}$ groups distribute in $P$ in Fig. 12. However, no definite evidence of such a relationship appears on this diagram: although the few points at $P>10-12 \mathrm{~d}$ all fall into the $M_{\text {core }} / M_{\star}=0$ group (and are therefore associated with larger dipole strengths, following Gregory et al. 2012), objects belonging to any $M_{\text {core }} / M_{\star}$ group are similarly mixed at shorter periods. To test this scenario further for the NGC 2264 sample, we examined the position of CTTS and WTTS on a H-R diagram with reference to their rotation properties; this is illustrated in Fig. 13. Again, no evolution in rotation properties is observed along the mass tracks, as objects evolve from being fully convective to developing a radiative core; shorter rotation periods (corresponding to violet/blue on the diagrams) and longer rotation periods (yellow/red) are well mixed across the depicted sample. This suggests that the internal structure has no obvious impact on the rotation properties of
Table 2. Subset of CTTS and relevant parameters used to test the disk locking assumptions.

\begin{tabular}{|c|c|c|c|c|c|}
\hline CSIMon-\# & $M_{\star}{ }^{1}$ & $R_{\star}^{1}$ & $\log \left(\dot{M}_{\mathrm{acc}}\right)^{1}$ & $M_{\text {core }} / M_{\star}^{2}$ & $P(d)$ \\
\hline 000007 & 0.69 & 2.4 & -7.22 & 0.00 & 3.192 \\
\hline 000153 & 0.29 & 1.4 & -8.04 & 0.00 & 1.896 \\
\hline 000290 & 0.25 & 3.4 & -7.53 & 0.00 & 5.900 \\
\hline 000314 & 0.29 & 1.4 & -8.02 & 0.00 & 3.279 \\
\hline 000326 & 0.66 & 1.3 & -8.77 & 0.03 & 6.642 \\
\hline 000358 & 0.29 & 1.4 & -8.18 & 0.00 & 5.821 \\
\hline 000406 & 1.13 & 1.3 & -7.32 & 0.81 & 6.631 \\
\hline 000412 & 0.45 & 2.2 & -6.98 & 0.00 & 6.679 \\
\hline 000433 & 0.44 & 1.0 & -8.86 & 0.00 & 9.798 \\
\hline 000484 & 0.13 & 0.3 & -10.04 & 0.00 & 19.50 \\
\hline 000619 & 0.69 & 1.4 & -7.40 & 0.01 & 6.404 \\
\hline 000637 & 0.45 & 1.6 & -8.50 & 0.00 & 12.31 \\
\hline 000717 & 0.53 & 2.0 & -7.55 & 0.00 & 8.558 \\
\hline 000766 & 0.53 & 1.5 & -7.67 & 0.00 & 2.798 \\
\hline 000926 & 0.40 & 1.3 & -8.30 & 0.00 & 12.32 \\
\hline 000964 & 0.95 & 1.5 & -8.43 & 0.23 & 3.289 \\
\hline 000965 & 0.36 & 1.7 & -7.99 & 0.00 & 9.688 \\
\hline 001003 & 0.24 & 1.5 & -8.70 & 0.00 & 3.454 \\
\hline 001054 & 0.36 & 2.2 & -6.68 & 0.00 & 8.142 \\
\hline 001114 & 0.40 & 2.4 & -7.37 & 0.00 & 2.579 \\
\hline 001132 & 0.33 & 1.6 & -6.55 & 0.00 & 2.958 \\
\hline 001187 & 0.40 & 1.5 & -8.72 & 0.00 & 3.102 \\
\hline 001199 & 1.20 & 1.9 & -7.69 & 0.01 & 3.617 \\
\hline 001217 & 1.30 & 1.8 & -7.05 & 0.52 & 7.865 \\
\hline 001234 & 0.94 & 1.7 & -8.84 & 0.10 & 9.606 \\
\hline 001249 & 0.30 & 3.0 & -7.53 & 0.00 & 1.954 \\
\hline 001294 & 0.92 & 1.0 & -7.68 & 0.87 & 6.723 \\
\hline 001296 & 0.69 & 2.0 & -8.10 & 0.00 & 9.725 \\
\hline 001308 & 0.63 & 1.6 & -7.72 & 0.00 & 6.717 \\
\hline 006079 & 0.45 & 1.9 & -8.12 & 0.00 & 0.511 \\
\hline 006325 & 0.54 & 1.0 & -8.59 & 0.06 & 0.956 \\
\hline 006465 & 0.88 & 1.0 & -7.88 & 0.88 & 2.829 \\
\hline
\end{tabular}

Notes. (1) Values from Venuti et al. (2014). Masses and radii are reported in units of $M_{\odot}$ and $R_{\odot}$, respectively. ${ }^{(2)}$ Estimate derived using the temperature and luminosity parameters derived for the object in Venuti et al. (2014) and Siess et al.'s (2000) model tracks.

young stars belonging to the NGC 2264 cluster. This conclusion, in contrast with the picture discussed in Gregory et al. (2012), may be a consequence of the youth of NGC 2264: at an age of $\sim 3 \mathrm{Myr}$, most of its members are still fully convective; therefore, the sample we are investigating here may be unsuitable for testing the connection between the evolution of magnetic topology and the rotational evolution of young stars.

\subsubsection{Testing magnetospheric accretion models}

Theories of magnetospheric accretion predict definite relationships between the stellar parameters $\left(M_{\star}, R_{\star}\right)$, the rotation pe$\operatorname{riod} P$, the magnetic field $B_{\star}$ and the accretion rate $\dot{M}_{\text {acc }}$. These predictions therefore provide an indirect way to test the validity of the magnetospheric accretion picture, when those same correlations are looked for among measured parameters for a given set of accreting young stars. Johns-Krull \& Gafford (2002) examined different theories of magnetospheric accretion, which in particular cover different assumptions on the geometry of the magnetic field. They collected a sample of a few tens of CTTS 


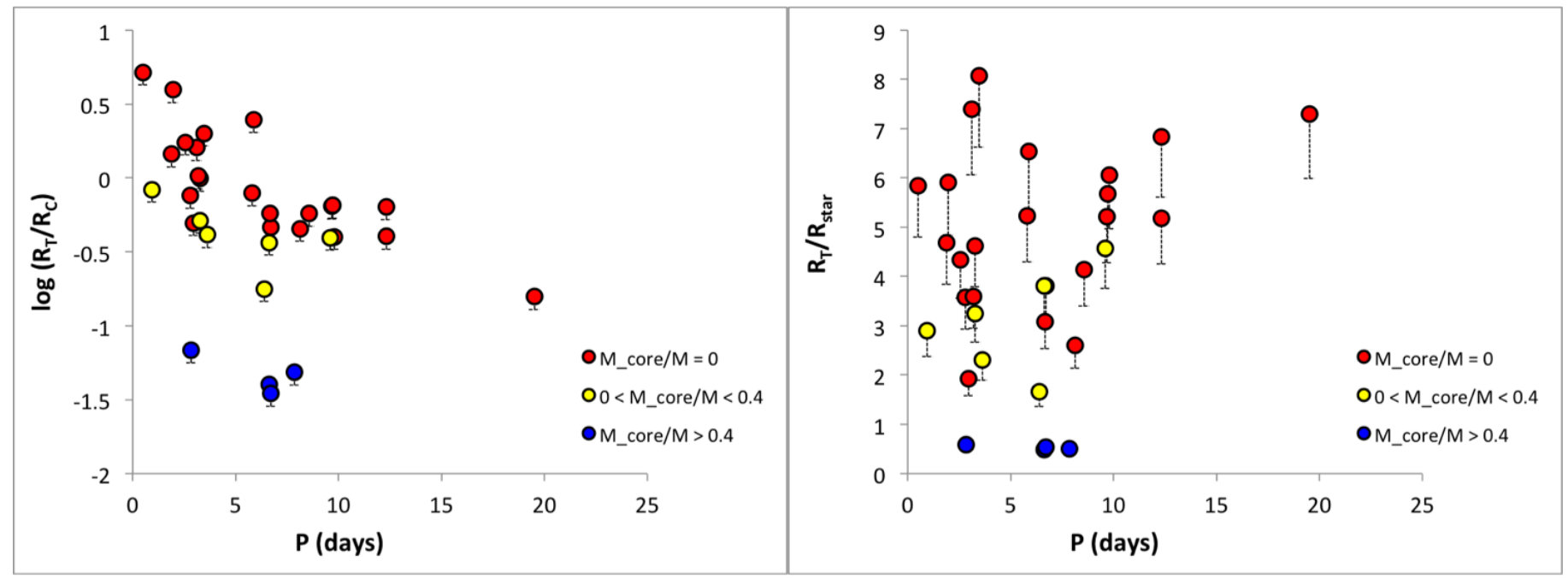

Fig. 12. Estimated ratios of truncation-to-corotation radius $\left(R_{\mathrm{T}} / R_{\mathrm{C}}, l e f t\right)$ and truncation-to-stellar radius $\left(R_{\mathrm{T}} / R_{\star}\right.$, right $)$, as a function of rotation period for a subsample of CTTS in NGC 2264 with period measurements from this study and stellar and accretion parameters measured from Venuti et al. (2014). The estimates of $R_{\mathrm{T}}$ and $R_{\mathrm{C}}$ are obtained following Bessolaz et al. (2008), in the assumption that steady accretion funnels are formed. Circles correspond to truncation radius estimates obtained assuming that the sonic Mach number at the disk midplane ( $\left.m_{\mathrm{S}}\right)$ is equal to 1 ; the lower bar associated with each point marks by how much the values of $R_{\mathrm{T}}$ or $R_{\mathrm{T}} / R_{\mathrm{C}}$ would vary if we adopted $m_{\mathrm{S}}=0.5$ (see Bessolaz et al. 2008). Red is used for objects with a mass ratio $M_{\text {core }} / M_{\star}$ between the radiative core and the total mass of the star equal to 0 (i.e., fully convective objects); yellow identifies objects in the $0<M_{\text {core }} / M_{\star}<0.4$ group; blue is for objects with $M_{\text {core }} / M_{\star}>0.4$. Different dipolar field strengths are used in the computation of $R_{\mathrm{T}}$ for each of these groups (see text).
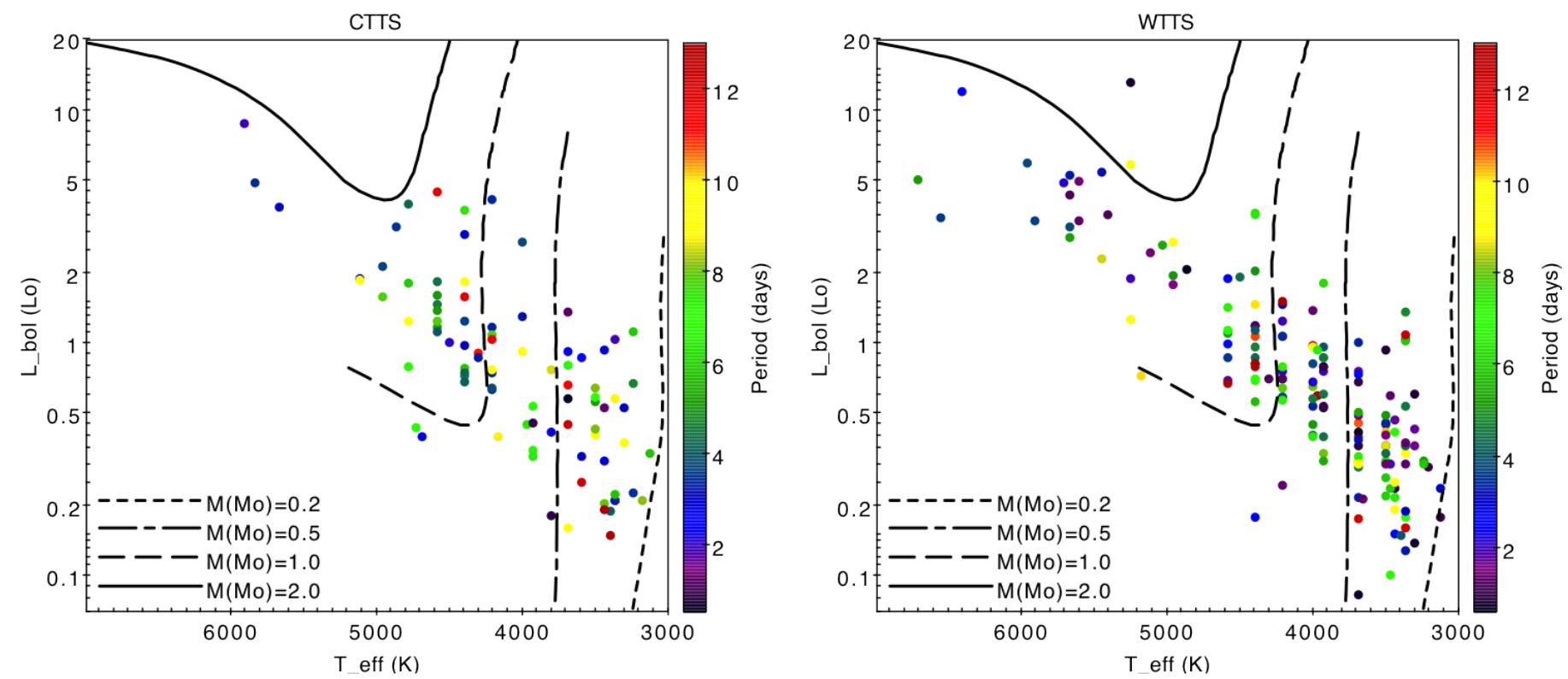

Fig. 13. Distribution, on the H-R diagram, of CTTS (left panel) and WTTS (right panel) in NGC 2264 with period measurements from the present study. A color scale, proportional to the period as indicated on the side axis to the right of the diagrams, is used to fill the symbols of each source. Mass tracks shown on the plots, truncated at an age of $30 \mathrm{Myr}$, are from Siess et al.'s (2000) evolutionary models.

with known stellar, rotation, and accretion parameters from the literature, and showed that the trend observed in the data best agrees with the predicted correlation of a modified version of the magnetospheric accretion theory presented in Ostriker \& Shu (1995), where the magnetic field topology is allowed to divert from a dipole. This predicted correlation is the following:

$R_{\star}^{2} f_{\mathrm{acc}} \propto M_{\star}^{1 / 2} \dot{M}_{\mathrm{acc}}^{1 / 2} P^{1 / 2}$,

where $f_{\text {acc }}$ is the filling factor of the accretion spots at the stellar surface, and it is assumed that the magnetic field strength $B_{\star}$ which participates in the accretion flow at the stellar surface does not vary significantly from object to object. Cauley et al. (2012) applied the same analysis to a sample of 36 CTTS in NGC 2264, and reached similar conclusions.

Here we perform the same test as in Cauley et al. (2012), for the subsample of NGC 2264 CTTS examined for Sect. 5.2.1. We follow Johns-Krull \& Gafford (2002) in assuming that $B_{\star}$ is the same for all stars in the sample, and adopt $M_{\star}, R_{\star}$ and $\dot{M}_{\text {acc }}$ values derived in Venuti et al. (2014) and individual estimates of $f_{\text {acc }}$ derived from spot modeling of simultaneous, multiwavelength light curves as described in Venuti et al. (2015). The values obtained, across our sample, for left and right side of Eq. (11), are compared in the upper panel of Fig. 14. Clearly, no 


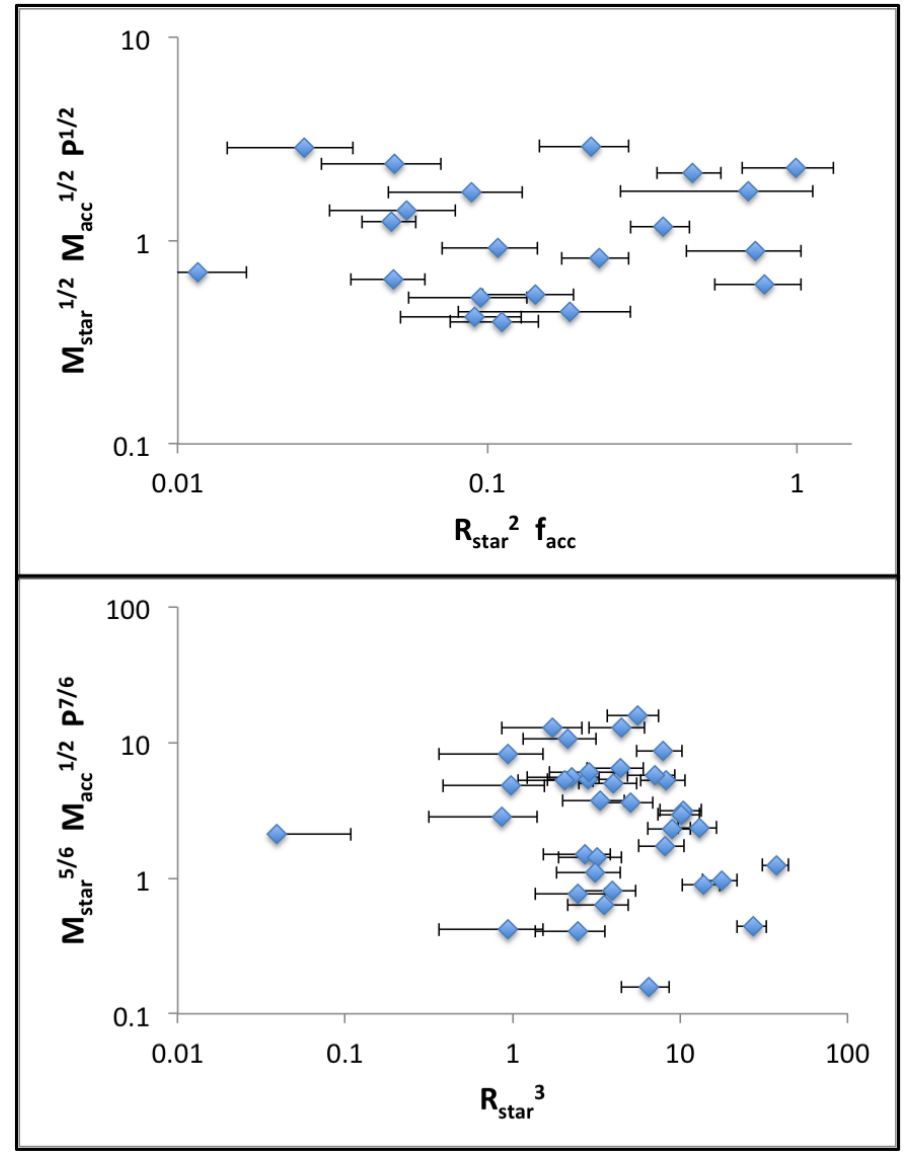

Fig. 14. Plot of left and right sides of Eq. (11) (upper panel) and Eq. (12) (lower panel), for a subset of NGC 2264 CTTS with known stellar and accretion parameters from Venuti et al. (2014) and rotation periods from this study.

significant correlation trend between the $x$-axis and the $y$-axis is present on the diagram. Most of the objects in the sample shown in Fig. 14 are fully convective $\left(M_{\text {core }} / M_{\star}=0\right.$, as deduced from the model tracks of Siess et al. 2000); therefore, the assumption that the $B_{\star}$ strength is uniform across the sample likely does not impact significantly this apparent lack of a correlation. A bigger impact on the position of individual points in Fig. 14 may arise from the values of $f_{\text {acc }}$, which, as discussed in Venuti et al. (2015), may be subject to somewhat large uncertainties when considered on an individual basis.

For completeness, we also tested whether similar prescriptions, extracted from magnetospheric accretion theories where the field is assumed to be purely dipolar (Königl 1991; Shu et al. 1994), would adapt better to the inferred parameters for NGC 2264 sources. In this case, the form of the expected correlation between $R_{\star}$ and $M_{\star}, \dot{M}_{\text {acc }}, P$ is the following:

$R_{\star}^{3} \propto M_{\star}^{5 / 6} \dot{M}_{\mathrm{acc}}^{1 / 2} P^{7 / 6}$,

where, again, we are assuming that the strength of the (dipolar) magnetic field can be considered to be the same for all sources. Left side and right side of Eq. (12) are plotted one against the other for NGC 2264 members in the lower panel of Fig. 14. Again, no correlation between the $x$-axis and the $y$-axis variables is found; however, the global behavior of objects on this diagram appears to be different from that in the upper panel of Fig. 14. In the first case (upper panel), points distribute along a fairly narrow, horizontal belt: they span a wide range of values along the $x$-axis, but show no apparent trend relative to the $y$-axis. Conversely, points form a diffuse cloud on the lower diagram in Fig. 14. This qualitative behavior is also observed in the analogous test diagrams shown in Johns-Krull \& Gafford (2002): data points tend to show a scatter plot on a $M_{\star}^{5 / 6} \dot{M}_{\text {acc }}^{1 / 2} P^{7 / 6}$ vs. $R_{\star}^{3}$ plane (whereas a correlation is predicted by Königl 1991 and Shu et al. 1994). On the other hand, a correlation trend between $R_{\star}^{2} f_{\text {acc }}$ and $M_{\star}^{1 / 2} \dot{M}_{\text {acc }}^{1 / 2} P^{1 / 2}$ is generally found by Johns-Krull \& Gafford (2002) when testing the predictions of Ostriker \& Shu's (1995) theory.

Error bars shown in Fig. 14 were obtained via error propagation on the relevant quantities, using a typical uncertainty of $0.2 R_{\odot}$ for $R_{\star}, 0.05 M_{\odot}$ for $M_{\star}, 0.5$ dex for $\dot{M}_{\text {acc }}$, and uncertainties derived in Venuti et al. (2015) for $f_{\text {acc }}$ and from Eq. (6) of this paper for the rotation period. Stellar radii appear to be the dominant source of uncertainty on these diagrams. The values of $R_{\star}$ adopted here were derived in Venuti et al. (2014) based on the effective temperatures $T_{\text {eff }}$ and bolometric luminosities $L_{\text {bol }}$ of the sources. $T_{\text {eff }}$ were assigned based on the spectral type of the objects and Cohen \& Kuhi's (1979) $\mathrm{SpT}-T_{\text {eff }}$ conversion scale; $L_{\text {bol }}$ were computed from the dereddened $J$-band photometry of the sources. An order-of-magnitude estimate of the uncertainty on the derived $R_{\star}$, taking into account typical uncertainties on the parameters involved in the computation of $R_{\star}$, is $\sim 0.1 R_{\odot}$. However, a comparison between the $R_{\star}$ estimates derived in Venuti et al. (2014) and those published in Rebull et al. (2002) results in a somewhat higher average discrepancy for objects in common. Therefore, we chose to adopt a more conservative value of $0.2 R_{\odot}$ for the error on $R_{\star}$ here. At any rate, this does not seem to impact significantly the trends observed on the diagrams in Fig. 14 and discussed in this section. Notably, uncertainties on $R_{\star}$ would affect the exact position of each point along the $x$-axis, while the overall range of values spanned by the point distribution along the two axes would remain mostly unaffected.

\section{The rotational evolution of young stars}

\subsection{The period distribution of the NGC 2264 cluster: multiple populations?}

As noted in Sect.4, the period distribution derived for the NGC 2264 cluster consists of a smooth distribution with two peaks $(P \sim 1-2 \mathrm{~d}$ and $P \sim 3-4 \mathrm{~d})$. Similar features are observed when analyzing the rotation periods separately for WTTS and CTTS, with the exception that, while WTTS do exhibit signatures of two peaks corresponding to those observed for the total period distribution, only the longer period peak $(P \sim 3-4 \mathrm{~d})$ is found in the case of CTTS. A fit to the different groups with a gamma distribution (see Sect. 4.1) provides the following parameters:

$$
\begin{aligned}
& \text { - cluster } \rightarrow \text { mean } P=5.2 \pm 0.6 \mathrm{~d} \text {; variance }=13 \pm 3 \mathrm{~d} \\
& \text { - CTTS } \rightarrow \text { mean } P=6.1 \pm 1.3 \mathrm{~d} \text {; variance }=13 \pm 4 \mathrm{~d} \\
& \text { - WTTS } \rightarrow \text { mean } P=4.9 \pm 0.8 \mathrm{~d} \text {; variance }=12 \pm 3 \mathrm{~d}
\end{aligned}
$$

The mean value measured for the whole sample falls in between the CTTS' mean period and the WTTS' mean period, and is closer to the latter than to the former, reflecting their relative contributions to the entire period distribution of the cluster. This shift between CTTS' and WTTS' period distributions can be understood if we consider the impact of star-disk interaction on the rotational properties and evolution of young stars. Given an ensemble of stars, it is reasonable to assume that their initial periods may be normally distributed (e.g., Tinker et al. 2002): the 
center of the distribution will reflect the average evolutionary status of the population; the dispersion of values around the center of the distribution will reflect varying initial conditions from object to object (e.g., varying star/disk mass, or different accretion history), which translate to varying rotational properties. As time progresses, if all objects follow similar evolutionary paths, we would then expect the period distribution of the ensemble of stars to evolve accordingly: the position of the center will change following the laws that govern angular momentum evolution in young stars; conversely, the overall shape of the distribution will remain unchanged (e.g., Vasconcelos \& Bouvier 2015). As WTTS represent a later evolutionary stage than CTTS, where the disk has disappeared and the systems are no longer braked by the star-disk interaction, the center of their period distribution is shifted toward shorter $P$.

The origin of the two peaks in Fig. 5 is more difficult to comprehend in this picture. As illustrated in the recent study of Vasconcelos \& Bouvier (2015), who simulated the rotational evolution of young low-mass stars from an age of 1 to $12 \mathrm{Myr}$, multiple peaks in the period distribution of a cluster at a given age can only be obtained if statistically distinct rotational behaviors are present $a b$ initio in the investigated population. If a homogeneous, Gaussian-shaped distribution of periods is assumed for the whole sample at time $t_{0}$ and this is let to evolve, a continuous distribution with a single peak will be obtained at time $t$. Therefore, the fact that we do observe two separate peaks may suggest that the sample of objects we are investigating comprises several subpopulations with different histories and rotational properties. This distinction goes beyond the separation between disk-bearing and disk-free sources, as one or two peaks on a smooth distribution of periods characterize both CTTS and WTTS individually.

Several studies have now assessed that star formation in NGC 2264 did not occur in a single event, but rather in a sequential fashion. Indeed, the presence of Herbig-Haro objects (e.g., Reipurth et al. 2004), molecular outflows (e.g., Margulis et al. 1988) and embedded sources (e.g., Wolf-Chase et al. 2003; Teixeira et al. 2006) attests that active star formation is still ongoing within the region. Sung et al. (2009) combined multiple investigations of the NGC 2264 cluster in the optical (Sung et al. 2008) and mid-IR (their study; Teixeira et al. 2006) to derive a map of the different subclusterings within the region, based on the spatial density of protostars and YSOs in various evolutionary stages (see Fig. 13 of their paper). In particular, they identified two embedded regions (the Spokes cluster and the core of the Cone nebula region, Cone $(\mathrm{C})$ ) in the southern part of the cloud, surrounded by a less embedded halo (Cone $(\mathrm{H}))$ dominated by more evolved YSOs. In the northern part of the cloud, another subclustering of disk-bearing YSOs is identified around the massive star S Mon. The S Mon and Cone $(\mathrm{H})$ subclusterings are in turn surrounded by the Halo region, which encompasses the periphery of the cloud and is prevalently populated by disk-free cluster members. As discussed in Sung \& Bessell (2010), objects located in the Halo were formed earlier, followed by objects in the $\mathrm{S}$ Mon region and in Cone $(\mathrm{H})$; objects in the Cone (C) and in the Spokes subclustering are the most recently formed.

To investigate the nature of objects that populate the $P=1-$ $2 \mathrm{~d}$ and the $P=3-4 \mathrm{~d}$ peaks, in Fig. 15 we compare the spatial distribution of objects belonging to these two period groups to that of the full sample of periodic members, and to the location of the various subclusters identified across NGC 2264 by Sung et al. (2009) and discussed above. The two period groups $\left(P=1-2 \mathrm{~d}\right.$ and $P=3-4 \mathrm{~d}$, hereafter $P^{[1,2]}$ and $\left.P^{[3,4]}\right)$ contain
Table 3. Distribution of periodic sources among the various NGC 2264 subregions.

\begin{tabular}{lcccc}
\hline \hline & Cone $(\mathrm{H})$ & S Mon & Halo & External \\
\hline Full sample & $29.8 \%$ & $28.0 \%$ & $27.8 \%$ & $14.4 \%$ \\
Periodic & $30.9 \%$ & $29.8 \%$ & $29.1 \%$ & $10.2 \%$ \\
$P^{[1,2]}$ & $38.5 \%$ & $25.6 \%$ & $28.2 \%$ & $7.7 \%$ \\
$P^{[3,4]}$ & $22.7 \%$ & $27.3 \%$ & $34.1 \%$ & $15.9 \%$ \\
\hline
\end{tabular}

Notes. Each column corresponds to a different subclustering identified in the NGC 2264 region by Sung et al. (2009) and illustrated here in Fig. 15. Rows indicate, in the order, the percentage of objects located on top of each of the subregions among (i) the full sample investigated in this study; (ii) objects found to be periodic (single period) in this study; (iii) periodic sources with period between 1 and 2 days; and (iv) periodic sources with period between 3 and 4 days. The percentage indicated for Cone $(\mathrm{H})$ also accounts for objects projected onto Cone $(\mathrm{C})$ and onto the Spokes region.

about the same number of objects (39 and 44, respectively). About $48 \%$ of objects in $P^{[1,2]}$ and $55 \%$ of objects in $P^{[3,4]}$ are in excess of the underlying continuum in the corresponding period bins (Fig. 5). To derive some quantitative indications of the spatial properties of objects in $P^{[1,2]}$ and of those in $P^{[3,4]}$ relative to the full sample of members investigated in this study and to the full sample of periodic sources identified in this study, for each of these groups we measured the fraction of objects projected onto the various subregions identified by Sung et al. (2009) within NGC 2264. These frequencies are compared in Table 3. About the same fraction of cluster members (30\%) are found projected on the S Mon, Cone (core + halo + Spokes cluster) and Halo regions. This percentage does not vary significantly if we restrict our sample of members to periodic sources only. Conversely, some quantitative difference in the spatial distribution of objects across the cloud can be observed when comparing the full sample of sources with objects in $P^{[1,2]}$ and $P^{[3,4]}$. Objects in the $P^{[1,2]}$ group appear to be more numerous at the RA-Dec location corresponding to the Cone region, and populate preferentially the sourthern part of the $S$ Mon region and the western part of the Halo region. On the other hand, a smallerthan-average fraction of objects in the $P^{[3,4]}$ group is projected on the Cone region, whereas they populate the periphery of the cloud and the Halo region more densely, and distribute predominantly along the eastern side of the latter. Interestingly, these are also the regions of the cluster where disks might be expected to last longer compared to more embedded regions, where the impact of the ionizing radiation from the OB stars contained in the cluster is stronger (see, e.g., the study of Mann \& Williams 2010 , on the ONC).

These features may support the view that several populations of stars are mingled with one another in the sample of cluster members that we are investigating here. However, no conclusive evidence in this respect can be drawn from this analysis. No obvious difference emerges between $P^{[1,2]}, P^{[3,4]}$ and the full sample of objects when comparing their respective distributions on the H-R diagram of the cluster, nor on their isochronal age distributions.

\subsection{NGC 2264 as a benchmark cluster in the scenario of PMS rotational evolution}

Recently, Bouvier et al. (2014) have presented a complete review of all observational studies of rotation in young stars 

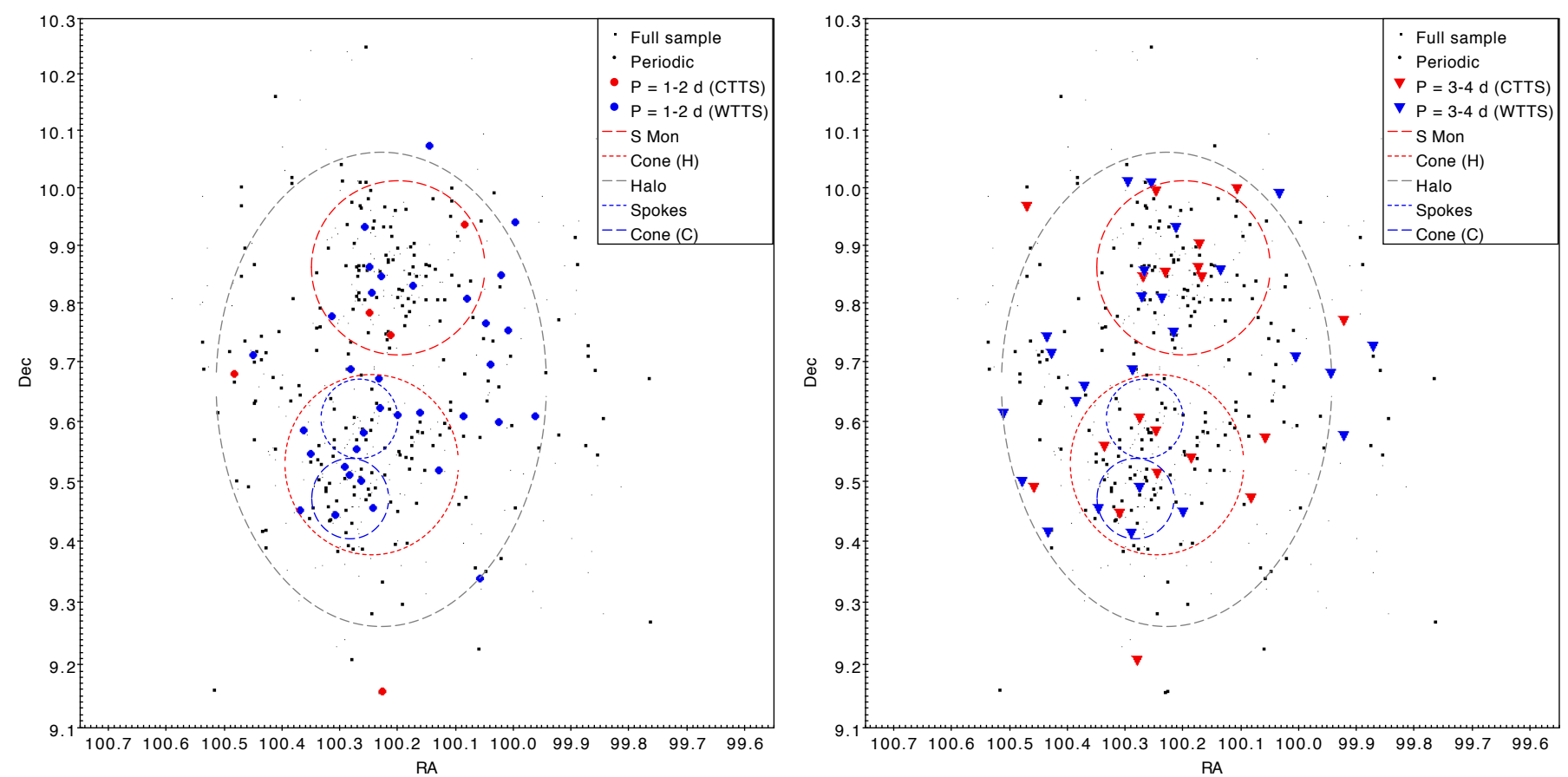

Fig. 15. Spatial distribution of NGC 2264 members investigated in this study. Small grey dots represent all objects in the sample (Table 4); periodic sources with single periodicity detected are highlighted as black dots; blue/red circles (on the left-side diagram) and triangles (right-side diagram) identify periodic sources with periods in the 1-2 d range and in the 3-4 d range, respectively; blue corresponds to WTTS, red to CTTS. The dashed lines delimit the various regions/subclusters identified, within the cloud, by Sung et al. (2009) after Sung et al. (2008) and Teixeira et al. (2006). North is up and east is left on the spatial diagram.

conducted so far on different clusters. To examine the rotation properties that we measure for NGC 2264 in the context of early stellar evolution, we select here all clusters from Bouvier et al.'s compilation, which have average ages between 1 and $15 \mathrm{Myr}$; this range defines the age scale of interest for the lifetimes of disks around young stars (e.g., Bell et al. 2013). Since we are interested here in a statistical comparison of rotation properties as a function of age, we have retained only clusters, in the age range mentioned earlier, in which rotation periods are available for a large statistical sample of members (as detailed in Table 1 of Bouvier et al. 2014). The selected clusters for our comparison are then, in order of age, NGC 6530, the ONC, Cepheus OB3b, NGC 2362, and h Persei. For these regions, we have then selected all members with known period and mass comprised between $0.1 M_{\odot}$ and $2 M_{\odot}$, i.e., approximately the mass range probed in our analysis of rotation in NGC 2264. The resulting period distributions for the various clusters, plus that obtained for NGC 2264 in this study and shown in Fig. 5, are shown in Fig. 16 in an age-ordered sequence.

A striking difference can be observed between the first panel (depicting NGC 6530, the youngest cluster in our sample) and the last panel (depicting h Per, the oldest cluster) in Fig. 16. As noted in Henderson \& Stassun (2012), the period distribution obtained for NGC 6530, at an age of a few Myr and a disk fraction $^{21}$ of about $50 \%$ (Prisinzano et al. 2007), appears to be uniform in the range $P=0-10$ days. Conversely, about $50 \%$ of the cluster members with detected periods in $\mathrm{h}$ Per (Moraux et al. 2013), at an age where the disk fraction has dropped to a very small percentage ( $\sim 2-3 \%$; Cloutier et al. 2014), exhibit rotation periods $\lesssim 1$ day. For clusters of intermediate ages, the period

\footnotetext{
${ }^{21}$ This corresponds to the percentage of cluster members with signatures of disks in the near-IR (i.e., exhibiting an IR excess at $J, H, K$ wavelengths).
}

distributions appear to exhibit transitional features between the first and the last panel in Fig. 16: a single or several peaks are observed in the $P=0-5$ day range, followed by a slow decline in number of objects toward larger periods.

\subsubsection{A mass dependence on the observational picture of rotational evolution?}

As discussed in Sect. 4.3, the results we obtain for NGC 2264 suggest that the rotation properties of cluster members are somewhat dependent on stellar mass. Fast rotators seem to be more predominant among lower-mass stars $\left(M_{\star}<0.4 M_{\odot}\right)$ than among higher-mass stars $\left(M_{\star}>1 M_{\odot}\right.$; see right panel of Fig. 6), although our data does not allow us to draw conclusive evidence in this respect from a statistical point of view. A similar analysis is presented by Littlefair et al. (2010) for the case of Cep OB3b. On the other hand, several studies conducted on other clusters have found a strong dependence of stellar rotation on stellar mass. Henderson \& Stassun (2012), for instance, reported a statistically significant difference in rotational periods between lower-mass $\left(M_{\star} \leq 0.5 M_{\odot}\right)$ and higher-mass $\left(M_{\star}>0.5 M_{\odot}\right)$ stars in NGC 6530 , with the latter rotating faster than the former. Conversely, a mass dependence in the opposite direction (lower-mass stars spinning on average faster than higher-mass stars) was reported for the ONC (Herbst et al. 2002) and for h Per (Moraux et al. 2013). As detailed in Table 1 of Bouvier et al. (2014), the various studies of rotation in young clusters, whose results we are comparing in Fig. 16, refer to mass regimes which may vary somewhat from case to case. This is illustrated in Fig. 17, showing the cumulative distribution functions in mass of the rotation surveys used to build Fig. 16. If the rotation properties of young stars are truly dependent on stellar mass, this diversity in mass properties between the different samples can 

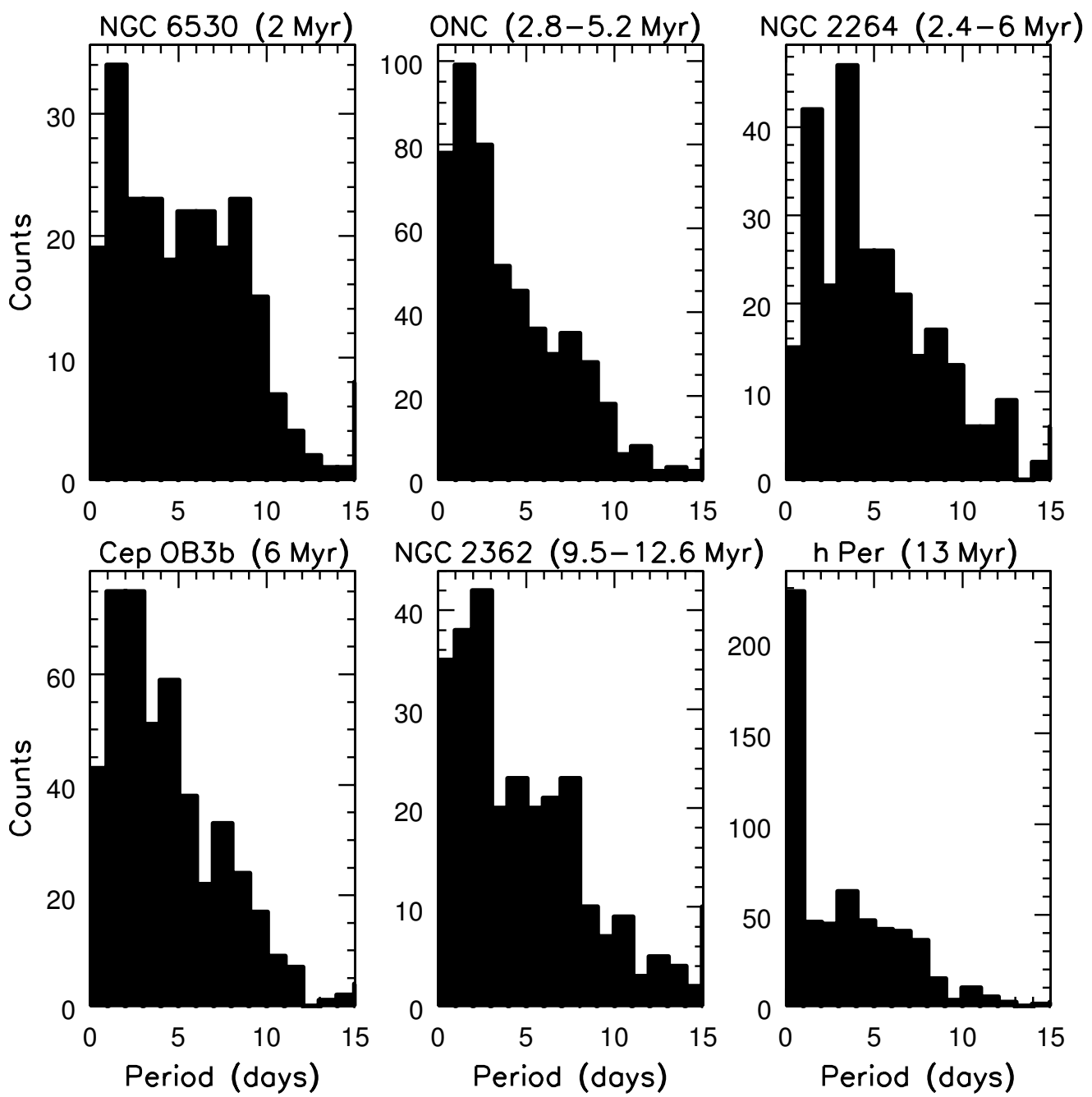

Fig. 16. Comparison of the period distributions obtained for various young clusters of different ages, in the mass range $0.1-2 M_{\odot}$. The clusters are, from left to right, from top to bottom: NGC 6530 (age of $~ 2 \mathrm{Myr}$, Bell et al. 2013; periods from Henderson \& Stassun $2012 ; 241$ objects with measured rotation periods in the mass range of interest here); the Orion Nebula Cluster (ONC; age in the range 2.8-5.2 Myr, Naylor 2009; rotation periods from Rodríguez-Ledesma et al. 2009 for objects with $M_{\star}<0.4 M_{\odot}$, and from Irwin \& Bouvier 2009, after Herbst et al. 2001,2002 and Stassun et al. 1999, for objects with $M_{\star}>0.4 M_{\odot} ; 528$ objects with measured periods in the selected mass range); NGC 2264 (age in the range 2.4-6 Myr, Naylor 2009; period measurements reported in this study; 272 objects); Cepheus OB3b (age of 6 Myr, Bell et al. 2013; periods from Littlefair et al. 2010; 460 objects with period measurements in the selected mass range); NGC 2362 (age in the range 9.5-12.6 Myr, Bell et al. 2013; periods from Irwin et al. 2008; 272 objects with periods in the mass range of interest); h Persei (age of 13 Myr, Mayne \& Naylor 2008; periods from Moraux et al. 2013; 586 objects with measured periods in the selected mass range).

have an impact on the evolutionary picture we may deduce from Fig. 16.

One way to circumvent this issue is to group cases with similar distributions in mass from Fig. 17, and compare the rotation properties as a function of age within each individual group. One such group consists of the ONC and Cep OB3b (masses from $\sim 0.1 M_{\odot}$ to $\sim 1.3 M_{\odot}$ ), whose rotation properties are illustrated respectively in the upper middle panel and in the lower left panel of Fig. 16. No definite qualitative difference is noted between the two period distributions. In both cases, the distribution has a single ${ }^{22}$ peak close to $P \sim 2 \mathrm{~d}$, perhaps more sharp in the case of the ONC, and then declines steadily toward longer

\footnotetext{
22 As mentioned in Sect. 4.3, Herbst et al. (2002) found the period distribution of the ONC to be unimodal when only objects less massive than $0.25 M_{\odot}$ are considered, and double-peaked when only objects more massive than $0.25 M_{\odot}$ are considered. The single-peaked nature of the period distribution shown here for the ONC reflects the fact that this is dominated by lower-mass stars: indeed, as illustrated in Fig. 17,
}

periods; in both regions, almost no objects exhibit rotational periods longer than $\sim 12 \mathrm{~d}$. It is important to mention that, while the Cep OB3b cluster (Kun et al. 2008) is a well defined subgroup of one of the three OB associations known in the Cepheus constellation, the ONC (Muench et al. 2008) likely comprises several different populations of stars, with a non-negligible age spread among cluster members. This ought to be taken into account when examining their respective period distributions as two distinct blocks in the picture of PMS rotational evolution.

A second group of cases with similar distributions in mass from Fig. 17 is that including NGC 6530 and NGC 2362. The corresponding period distributions are shown on the upper left panel and on the lower middle panel of Fig. 16, respectively. Contrary to the case of the ONC and Cep OB3b, a marked age difference exists between these two clusters: at an age of about 2 Myr, NGC 6530 is the youngest cluster among those shown in

about $50 \%$ of objects in the ONC sample considered here have mass below $0.3 M_{\odot}$. 


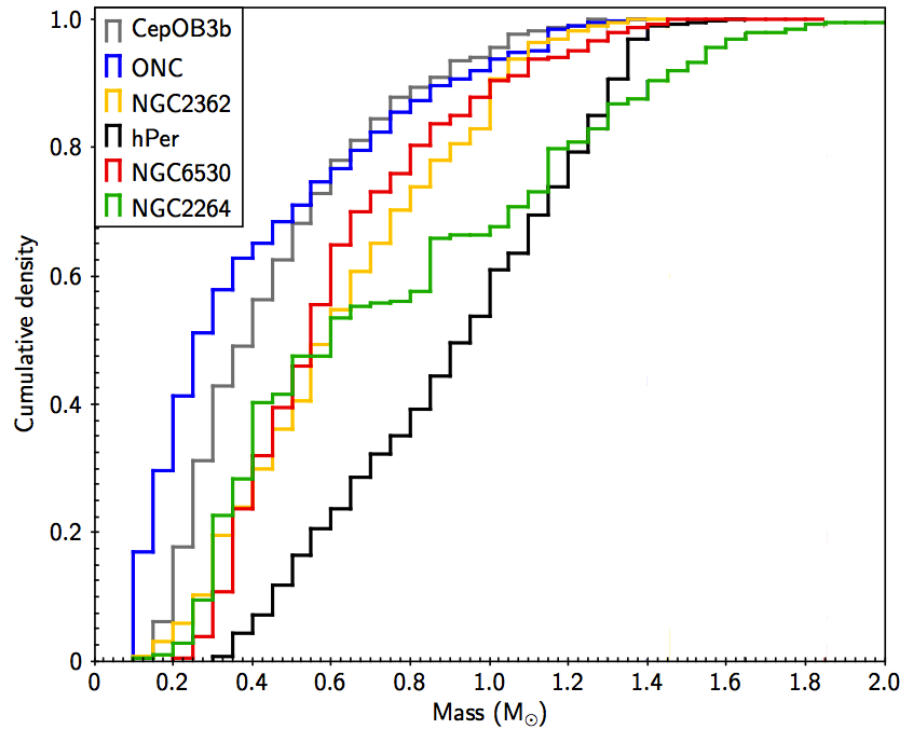

Fig. 17. Cumulative distribution functions, in mass, of the young stellar populations whose rotation properties are used to build the histograms of periods shown in Fig. 16 for the various clusters.

Fig. 16 and infrared studies indicate that about half of its members are surrounded by dusty disks; conversely, at an average age of about $12 \mathrm{Myr}$, only 10-20\% of objects in the NGC 2362 cluster show evidence of dust in the circumstellar environment (Dahm \& Hillenbrand 2007). The overall shape of the period distribution appears to evolve between the two: only a hint of a peak around $P=1-2 \mathrm{~d}$ is present in the case of NGC 6530, and the distribution is fairly uniform in the $\sim 1-10 \mathrm{~d}$ period range; conversely, in the case of NGC 2362 a more definite peak around $P \sim 2 \mathrm{~d}$ is present and stands out against the flat segment of distribution in the 3-8 d period range, after which the distribution displays a rapidly declining tail toward longer periods.

To investigate the impact of different mass regimes on the global picture of Fig. 16, we used Fig. 17 to identify a mass range common to all samples. Then, we selected, for each cluster, only objects with masses in this range, and used these mass-selected subsamples of objects to re-draw the period distributions in Fig. 16. The selected mass range goes from $0.4 M_{\odot}$ (low-mass end of the $\mathrm{h}$ Per sample) to $1.1 M_{\odot}$ (chosen a bit smaller than the highest mass regime common to all samples, $\sim 1.3 M_{\odot}$, to avoid the mass range where the so-called "Kraft break" in stellar rotation properties occurs; see Kraft 1967). The results of this exercise are illustrated in Fig. 18.

When comparing Figs. 16 and 18 qualitatively, the following differences appear:

- in the case of NGC 6530 (2 Myr), the net effect of this mass selection on the global shape of the period distribution is a removal of objects from the intermediate (7-9 d) period range, with a steadier decline in number, instead of a uniform behavior, for $P \gtrsim 7 \mathrm{~d}$;

- in the case of the ONC (2.8-5.2 Myr), a second peak at $P \sim$ 7-8 d appears in the period distribution when $M_{\star}<0.4 M_{\odot}$ objects are excluded, in agreement with what reported by Herbst et al. (2002) regarding a bimodal nature of the period distribution for the more massive population component of the star-forming region;

- in the case of NGC 2264 (2.4-6 Myr), more substructures emerge, notably a hint of a peak at $P \sim 6-7 \mathrm{~d}$, and in addition the first peak of the period distribution $(P=1-2 \mathrm{~d})$ becomes less pronounced with respect to its second peak $(P=3-4 \mathrm{~d})$ compared to the histogram shown in Fig. 5;

- in the case of Cep OB3b (6 Myr), the net effect of this mass selection is sharpening the peak of the distribution, which becomes more populated in the higher- $P(2-3 \mathrm{~d})$ half than in the lower- $P(1-2 \mathrm{~d})$ half;

- in the case of NGC 2362 (9.5-12.6 Myr), when removing the $M_{\star}<0.4 M_{\odot}$ objects, the peak at $P \sim 2 \mathrm{~d}$ disappears and the shape of the distribution evolves toward a uniform distribution in the $P=0-8 \mathrm{~d}$ range;

- in the case of h Per (13 Myr), no appreciable changes in the shape of the period distribution appear when restricting the sample to more massive objects.

The above list suggests that, at least in the age range between 3 and $10 \mathrm{Myr}$, the rotation properties of young star clusters are somewhat dependent on the mass regimes probed in their populations: when restricting the sample to more massive TTS $\left(0.4-1.1 M_{\odot}\right)$, the features at longer periods in the cluster distributions become more marked than when lower-mass TTS $\left(M_{\star}<0.4 M_{\odot}\right)$ are considered. Conversely, no significant variations in the evolutionary picture emerge when comparing the age-ordered sequence of period histograms in Fig. 16 to that in Fig. 18. From the youngest (NGC 6530) to the most evolved (h Per) case, the period distribution evolves from a uniform distribution in the $\sim 0-8 \mathrm{~d}$ period range (with perhaps a hint of a peak at a few days) to a distribution with a sharp peak at $P<1 \mathrm{~d}$ superimposed on a flat continuum, about five times less strong than the peak, that extends down to $P \sim 8 \mathrm{~d}$. At intermediate stages, the period distributions of young clusters exhibit a variety of features and substructures in the period range from 0 to $10 \mathrm{~d}$, which may reflect a diversity in the specific environmental conditions or in the nature of the stellar populations probed in the different cases. Overall, the bulk of objects is found at periods shorter than $\sim 7$ days, and the distribution declines more or less steadily toward longer periods.

\subsubsection{The rotational evolution of young stars: observations vs. simulations}

The wealth of observational data available to date for rotation periods of young stars in cluster of different ages have enabled a number of studies that follow a semi-empirical approach to model the rotational evolution of stars in the pre-main sequence. Namely, these models take a starting population of stars whose rotation properties are assigned based on observations of the youngest clusters, and follow their rotational evolution assuming that this is governed by specific physical mechanisms (e.g., disk-locking, angular momentum conservation) depending on the presence or absence of accretion disks. The comparison of the final period distribution simulated with observational data for clusters of similar age therefore enables investigating what processes regulate the spin evolution of young stars, especially in the earliest stages.

Vasconcelos \& Bouvier (2015) presented Monte Carlo simulations of the rotational evolution of a population of 280000 young stars with mass between 0.3 and $1 M_{\odot}$, from an age of 1 Myr to an age of $12 \mathrm{Myr}$. Their models assume that stars evolve at a constant angular velocity when they are coupled to an active accretion disk, and at a constant angular momentum when the disk is dissipated. Their Fig. 6 illustrates how the shape of the global period distribution evolves from the beginning to the end of the simulated time span. It is assumed that, at an age of $1 \mathrm{Myr}$, a fraction of young stars have already lost their disks, 

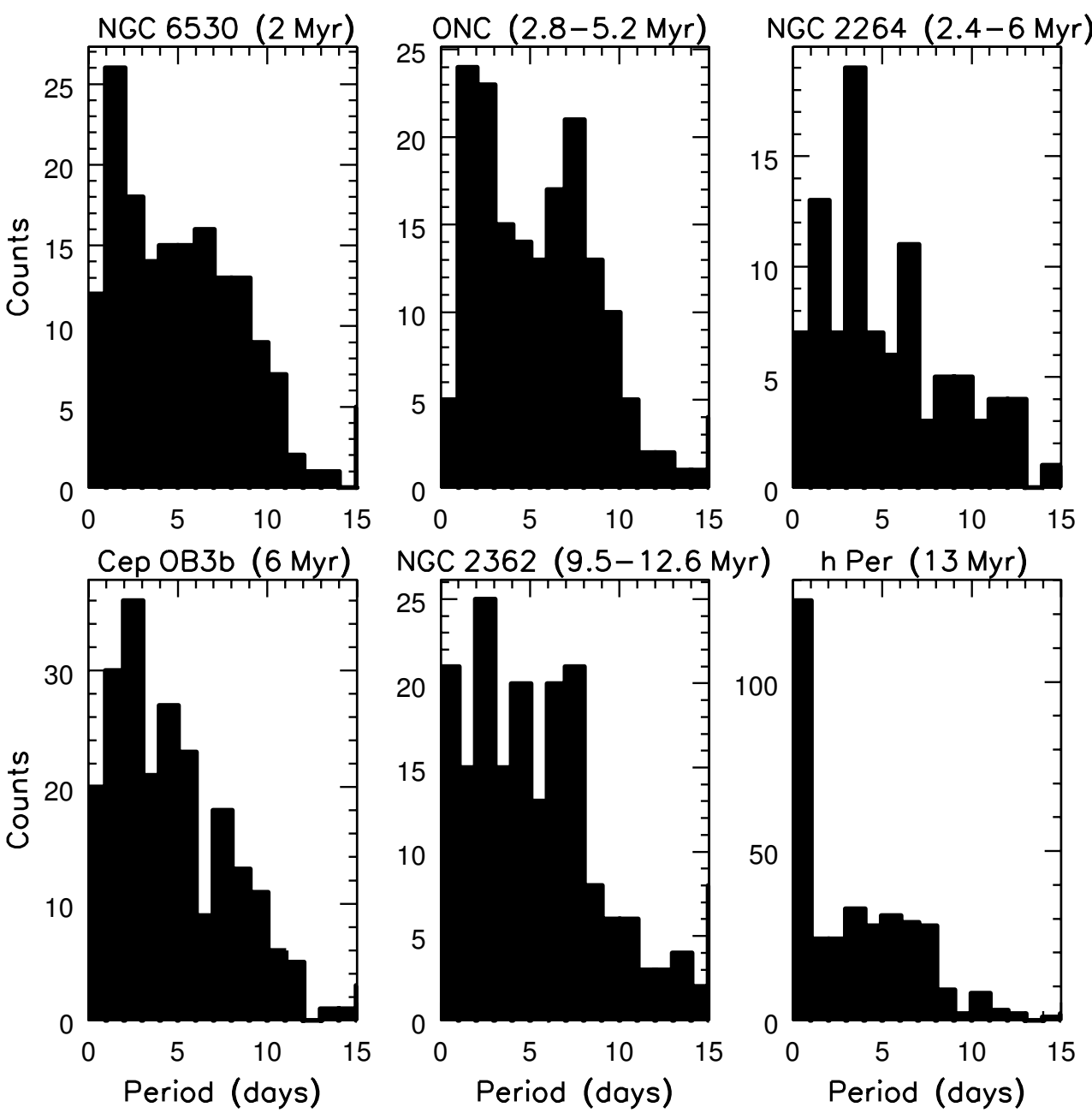

Fig. 18. Same as in Fig. 16, but including only objects in the mass range $0.4-1.1 M_{\odot}$ for each cluster.

and that the rotation properties of the latter are statistically distinct from those of disk-bearing sources; this is consistent with what observed in several young clusters (e.g., Xiao et al. 2012; Henderson \& Stassun 2012). At an age of 2.1 Myr, the initial distribution, with a broad "bump" around a period of $3 \mathrm{~d}$ and a slow decline toward longer periods, has evolved into a distribution with a more pronounced peak around $P \sim 2$ d followed by a flatter region, about half as high as the peak, that extends from 5 to $10 \mathrm{~d}$ and then declines steadily toward longer periods. This behavior can reproduce qualitatively the shape of the period distribution observed for the ONC and illustrated in the upper-middle panel of Fig. 16. By an age of 12.1 Myr, the peak in the distribution has become sharp and shifted to $P \lesssim 1 \mathrm{~d}$, while the number count in the successive histogram bin drops to about 0.4 times that in the peak and decreases steadily with increasing period. This trend is similar, at least on a qualitative basis, to what is observed for the NGC 2362 and the more evolved h Per clusters (lower-middle and lower-right panel of Fig. 16, respectively).

At an age of $\sim 3-5$ Myr (on the order of disk lifetime; Haisch et al. 2001), NGC 2264 is a benchmark cluster for PMS rotational evolution. By that time, about $50 \%$ of young stars will have lost their disks and started to spin up toward the main sequence. An implication of this is that, at the evolutionary stage of NGC 2264, a fraction of objects will have just been released from their disks, and therefore will not have had enough time yet to spin up significantly. As discussed in Vasconcelos \& Bouvier (2015), during the first few Myr of evolution of a given ensemble of stars, the progressive release of YSOs from their disks results in a widening of the period distribution associated with the diskfree component of the population: newly released objects will rotate more slowly than stars that have lost their disks earlier and have thus already started to spin up freely. This may explain the tail of slow rotators observed among WTTS in NGC 2264 (Fig. 6). As the evolution continues, more and more sources are released from their disks and stars that had been released earlier keep spinning up; therefore, the bulk of the non-accreting population shifts toward shorter periods and the long- $P$ region of the initial distribution is depleted. This is consistent with the picture shown in Fig. 16, where clusters at intermediate evolutionary stages exhibit wider distributions at the longer period end than the h Per cluster.

As discussed above, the predicted trend of spin rate evolution of young stellar populations, simulated in Vasconcelos \& Bouvier (2015), shows an overall agreement with the evolutionary picture we may get from comparing the observed period distributions of clusters at different ages (Fig. 16 of this study). This supports the view that young stars may be locked to their disks during the earliest stages of their evolution, and then spin up as they contract toward the ZAMS once the magnetic coupling with their accretion disks has ceased. Aligned with this interpretation are the conclusions of Landin et al. (2016), who tested the idea, put forward by Lamm et al. (2005), that NGC 2264 represents a later stage in the scenario of PMS rotational evolution than the ONC. The authors simulated the backward evolution of 
the NGC 2264 period distribution down to the age of the ONC, under the assumption that the spin rate of cluster members is governed by disk locking as long as the stars possess a disk, and by angular momentum conservation afterwards. The period distribution predicted by Landin et al. (2016) for the younger NGC 2264, following this approach, would indeed show the overall features of the period distribution observed for the ONC.

\section{Conclusions}

In this study we have presented the most accurate and unbiased analysis of rotation properties available to date for the NGC 2264 cluster in the mass range $\sim 0.2-1.7 M_{\odot}$. We examined a population of about 500 cluster members, whose optical light variations were monitored continuously for 38 days with the CoRoT space telescope in the framework of the CSI 2264 campaign (Dec. 2011-Jan. 2012). Light curves were searched for periodicity using three different methods: the Lomb-Scargle periodogram, the autocorrelation function, and the string-length method. A significant period was detected for about $62 \%$ of sources in the sample; the period detection rate is lower among objects with active accretion disks (CTTS) than among objects that have already been released from their disks (WTTS).

The main results of this work can be summarized as follows.

1. The period distribution derived for the cluster consists of a smooth distribution centered on $P \sim 5.2 \mathrm{~d}$ with two peaks. The peaks are located at $P=1-2 \mathrm{~d}$ and $P=3-4 \mathrm{~d}$. Although our dataset allows us to reliably measure rotation periods as long as $19 \mathrm{~d}$, over $95 \%$ of periodic sources in our sample have period shorter than $13 \mathrm{~d}$.

2. A clear statistical distinction in rotation properties exists between WTTS and CTTS: although the respective period distributions overlap, the former spin on average faster than the latter. A typical period of $4.9 \mathrm{~d}$ is measured across the WTTS population of the cluster, while the mean period measured for the CTTS sample is of $6.1 \mathrm{~d}$. The first peak in the NGC 2264 period distribution $(P=1-2 \mathrm{~d})$ is clearly associated with its WTTS population, whereas very few CTTS are found with $P<2.5 \mathrm{~d}$; conversely, the second peak, at $P=3-4 \mathrm{~d}$, takes contributions from both CTTS and WTTS.

3. Our results suggest some mass dependence in the rotation properties of NGC 2264 members, in agreement with earlier findings. Lower-mass objects appear to exhibit rotation periods that are shorter on average than higher-mass objects, although our analysis does not allow us to reject the null hypothesis that lower-mass and higher-mass objects have similar period distributions to the 5\% significance level.

4. A clear connection is found between rotation and accretion; objects that exhibit large UV excesses (indicative of high mass accretion rates onto the stars) are typically associated with long rotation periods; conversely, a dearth of fast rotators with strong UV excesses is evident among disk-bearing objects. This supports the idea that magnetic star-disk coupling has an impact on the rotation properties of young stars.

5. No clear relationship emerges between the rotation period of the stars and their inner structure (notably the presence/absence of a radiative core); no evolution in rotation properties is observed along a given mass track on the $\mathrm{H}-\mathrm{R}$ diagram of the cluster among CTTS or WTTS. This may indicate that the NGC 2264 population is still too young for the transition from fully convective to partly radiative stellar interiors to have a significant impact on the observed properties.

6 . The connection between rotation properties and accretion indicators (UV excess) that we find here for the NGC 2264 population is reminiscent of the connection between rotation and disk indicators (IR excess) in young stars reported in Rebull et al. (2006). Furthermore, it shows the same behavior of the distribution of accretion rates as a function of rotation period simulated in Vasconcelos \& Bouvier (2015) in the hypothesis that the spin rate of young stars evolves at constant angular velocity in the presence of a disk and at constant angular momentum when the disk has disappeared. This would support a scenario in which young stars are locked to their disks during the accretion phase and then start to spin up to conserve angular momentum once disk accretion and star-disk coupling have ceased.

Thanks to the extensive obervational effort devoted to characterizing the period distributions of star clusters at different ages, and to the simultaneous modeling effort aimed at reproducing and interpreting those observations, we have now achieved a global understanding of how the stellar spin rate may evolve across the pre-main sequence. However, several open issues remain. For instance, it is not clear why the period distributions of some clusters exhibit two separate peaks, as is the case for NGC 2264. In this study, we show that objects in the two peaks observed for NGC 2264 are different in nature: the shorter-period peak consists of disk-free cluster members (WTTS), while the longerperiod peak consists of both disk-accreting (CTTS) and disk-free cluster members. However, the origin of these peaks is not as clear. Notably, they appear to be additional features on top of an underlying, smooth distribution of periods; this behavior is observed both when considering the NGC 2264 cluster as a whole and when examining CTTS and WTTS separately. Are the initial rotation periods in a given population randomly distributed (in which case we would expect the period distribution to evolve with time into another random distribution where the center has shifted toward shorter values)? And, therefore, is the presence of multiple peaks indicative of the fact that the ensemble of stars under exam is a composite population (e.g., the result of distinct star formation episodes)? Or do the initial rotation periods in a given sample of objects cluster around a certain value, depending on, e.g., environmental conditions at birth? Significant contribution to this discussion may be provided in the near future by Gaia: data issued from the mission will help identify kinematical substructures and populations in NGC 2264, which may shed new light on the nature of the specific features seen in the rotational distribution of the cluster.

Acknowledgements. We thank the referee for a prompt and constructive report. This work is based on data from the CoRoT space mission, which has been developed and operated by CNES, with the contribution of Austria, Belgium, Brazil, ESA (RSSD and Science Programme), Germany, and Spain. This publication also makes use of data from MegaPrime/MegaCam, a joint project of CFHT and CEA/DAPNIA, at the Canada-France-Hawaii Telescope (CFHT) which is operated by the National Research Council (NRC) of Canada, the Institut National des Sciences de l'Univers of the Centre National de la Recherche Scientifique (CNRS) of France, and the University of Hawaii. We thank Suzanne Aigrain for detrending the CoRoT light curves used in this work. L.V. acknowledges useful discussions on histogram statistics with Dipan Sengupta. The authors acknowledge support through the PRIN INAF 2014 funding scheme of the National Institute for Astrophysics (INAF) of the Italian Ministry of Education, University and Research ("The GAIA-ESO Survey", P.I.: S. Randich). This study was also supported by the grant ANR 2011 Blanc SIMI5-6 02001 "Toupies: Towards understanding the spin evolution of stars". S.H.P.A. acknowledges financial support from CNPq, CAPES and Fapemig. 


\section{References}

Affer, L., Micela, G., Favata, F., \& Flaccomio, E. 2012, MNRAS, 424, 11 Affer, L., Micela, G., Favata, F., Flaccomio, E., \& Bouvier, J. 2013, MNRAS, 430,1433

Artemenko, S. A., Grankin, K. N., \& Petrov, P. P. 2012, Astron. Lett., 38, 783

Attridge, J. M., \& Herbst, W. 1992, ApJ, 398, L61

Baglin, A., Auvergne, M., Barge, P., et al. 2006, in The CoRoT Mission PreLaunch Status - Stellar Seismology and Planet Finding, eds. M. Fridlund, A. Baglin, J. Lochard, \& L. Conroy, ESA SP, 1306, 33

Baraffe, I., Chabrier, G., \& Gallardo, J. 2009, ApJ, 702, L27

Baxter, E. J., Covey, K. R., Muench, A. A., et al. 2009, AJ, 138, 963

Bell, C. P. M., Naylor, T., Mayne, N. J., Jeffries, R. D., \& Littlefair, S. P. 2013, MNRAS, 434, 806

Bessell, M. S., Castelli, F., \& Plez, B. 1998, A\&A, 333, 231

Bessolaz, N., Zanni, C., Ferreira, J., Keppens, R., \& Bouvier, J. 2008, A\&A, 478, 155

Bodenheimer, P. 1995, ARA\&A, 33, 199

Bouvier, J., Bertout, C., Benz, W., \& Mayor, M. 1986, A\&A, 165, 110

Bouvier, J., Cabrit, S., Fernandez, M., Martin, E. L., \& Matthews, J. M. 1993 A\&A, 272, 176

Bouvier, J., Alencar, S. H. P., Boutelier, T., et al. 2007a, A\&A, 463, 1017

Bouvier, J., Alencar, S. H. P., Harries, T. J., Johns-Krull, C. M., \& Romanova, M. M. 2007b, Protostars and Planets V, 479

Bouvier, J., Matt, S. P., Mohanty, S., et al. 2014, Protostars and Planets VI, 914 433

Box, G. E. P., \& Jenkins, G. M. 1976, Time series analysis. Forecasting and control (San Francisco: Holden-Day)

Cauley, P. W., Johns-Krull, C. M., Hamilton, C. M., \& Lockhart, K. 2012, ApJ, 756, 68

Choi, P. I., \& Herbst, W. 1996, AJ, 111, 283

Cieza, L., \& Baliber, N. 2006, ApJ, 649, 862

Cieza, L., \& Baliber, N. 2007, ApJ, 671, 605

Cloutier, R., Currie, T., Rieke, G. H., et al. 2014, ApJ, 796, 127

Cody, A. M., Stauffer, J., Baglin, A., et al. 2014, AJ, 147, 82

Cohen, M., \& Kuhi, L. V. 1979, ApJS, 41, 743

Dahm, S. E. 2008, in Handbook of Star Forming Regions, Volume I, ed. B. Reipurth, 966

Dahm, S. E., \& Hillenbrand, L. A. 2007, AJ, 133, 2072

Davies, C. L., Gregory, S. G., \& Greaves, J. S. 2014, MNRAS, 444, 1157

DeCarlo, L. T. 1997, Psychol. Meth., 292

Donati, J.-F., Skelly, M. B., Bouvier, J., et al. 2010, MNRAS, 409, 1347

Dworetsky, M. M. 1983, MNRAS, 203, 917

Eaton, N. L., Herbst, W., \& Hillenbrand, L. A. 1995, AJ, 110, 1735

Edwards, S., Strom, S. E., Hartigan, P., et al. 1993, AJ, 106, 372

Ekström, S., Meynet, G., Maeder, A., \& Barblan, F. 2008, A\&A, 478, 467

Fallscheer, C., \& Herbst, W. 2006, ApJ, 647, L155

Fürész, G., Hartmann, L. W., Szentgyorgyi, A. H., et al. 2006, ApJ, 648, 1090

Flaccomio, E., Micela, G., Sciortino, S., et al. 2005, ApJS, 160, 450

Freedman, D., \& Diaconis, P. 1981, Z. Wahrscheinlichkeitstheorie und verwandte Gebiete, 57, 453

Gallet, F., \& Bouvier, J. 2013, A\&A, 556, A36

Ghosh, P., \& Lamb, F. K. 1978, ApJ, 223, L83

Gillen, E., Aigrain, S., McQuillan, A., et al. 2014, A\&A, 562, A50

Grankin, K. N., Melnikov, S. Y., Bouvier, J., Herbst, W., \& Shevchenko, V. S. 2007, A\&A, 461, 183

Grankin, K. N., Bouvier, J., Herbst, W., \& Melnikov, S. Y. 2008, A\&A, 479, 827

Gregory, S. G., Donati, J.-F., Morin, J., et al. 2012, ApJ, 755, 97

Haisch, Jr., K. E., Lada, E. A., \& Lada, C. J. 2001, ApJ, 553, L153

Hartigan, J. A., \& Hartigan, P. M. 1985, Ann. Stat., 13, 70

Hartigan, P. M. 1985, J. R. Stat. Soc. Ser. C, 34, 320

Hartmann, L., \& Stauffer, J. R. 1989, AJ, 97, 873

Henderson, C. B., \& Stassun, K. G. 2012, ApJ, 747, 51

Herbig, G. H. 1962, Adv. Astron. Astrophys., 1, 47

Herbig, G. H., \& Bell, K. R. 1988, Third Catalog of Emission-Line Stars of the Orion Population, 3

Herbst, W., \& Mundt, R. 2005, ApJ, 633, 967

Herbst, W., Herbst, D. K., Grossman, E. J., \& Weinstein, D. 1994, AJ, 108, 1906

Herbst, W., Bailer-Jones, C. A. L., \& Mundt, R. 2001, ApJ, 554, L197

Herbst, W., Bailer-Jones, C. A. L., Mundt, R., Meisenheimer, K., \& Wackermann, R. 2002, A\&A, 396, 513

Herbst, W., Eislöffel, J., Mundt, R., \& Scholz, A. 2007, Protostars and Planets V, 297

Horne, J. H., \& Baliunas, S. L. 1986, ApJ, 302, 757

Irwin, J., \& Bouvier, J. 2009, in IAU Symp. 258, eds. E. E. Mamajek, D. R. Soderblom, \& R. F. G. Wyse, 363

Irwin, J., Hodgkin, S., Aigrain, S., et al. 2008, MNRAS, 384, 675

Johns-Krull, C. M., \& Gafford, A. D. 2002, ApJ, 573, 685
Johnstone, C. P., Jardine, M., Gregory, S. G., Donati, J.-F., \& Hussain, G. 2014, MNRAS, 437, 3202

Joy, A. H. 1945, ApJ, 102, 168

Königl, A. 1991, ApJ, 370, L39

Kraft, R. P. 1967, ApJ, 150, 551

Kulkarni, A. K., \& Romanova, M. M. 2008, MNRAS, 386, 673

Kun, M., Kiss, Z. T., \& Balog, Z. 2008, Star Forming Regions in Cepheus, ed. B. Reipurth, 136

Kurosawa, R., \& Romanova, M. M. 2013, MNRAS, 431, 2673

Lamm, M. H., Bailer-Jones, C. A. L., Mundt, R., Herbst, W., \& Scholz, A. 2004, A\&A, 417, 557

Lamm, M. H., Mundt, R., Bailer-Jones, C. A. L., \& Herbst, W. 2005, A\&A, 430, 1005

Landin, N. R., Mendes, L. T. S., Vaz, L. P. R., \& Alencar, S. H. P. 2016, A\&A, 586, A96

Littlefair, S. P., Naylor, T., Mayne, N. J., Saunders, E. S., \& Jeffries, R. D. 2010, MNRAS, 403, 545

Luhman, K. L., Stauffer, J. R., Muench, A. A., et al. 2003, ApJ, 593, 1093

Makidon, R. B., Rebull, L. M., Strom, S. E., Adams, M. T., \& Patten, B. M 2004, AJ, 127, 2228

Manara, C. F., Rosotti, G., Testi, L., et al. 2016, A\&A, 591, L3

Mann, R. K., \& Williams, J. P. 2010, ApJ, 725, 430

Margulis, M., Lada, C. J., \& Snell, R. L. 1988, ApJ, 333, 316

Matt, S., \& Pudritz, R. E. 2005, ApJ, 632, L135

Matt, S. P., Pinzón, G., Greene, T. P., \& Pudritz, R. E. 2012, ApJ, 745, 101

Mayne, N. J., \& Naylor, T. 2008, MNRAS, 386, 261

McGinnis, P. T., Alencar, S. H. P., Guimarães, M. M., et al. 2015, A\&A, 577, A11

McQuillan, A., Aigrain, S., \& Mazeh, T. 2013, MNRAS, 432, 1203

Mighell, K. J., \& Plavchan, P. 2013, AJ, 145, 148

Moraux, E., Artemenko, S., Bouvier, J., et al. 2013, A\&A, 560, A13

Muench, A., Getman, K., Hillenbrand, L., \& Preibisch, T. 2008, Star Formation in the Orion Nebula I: Stellar Content, ed. B. Reipurth, 483

Muzerolle, J., Calvet, N., \& Hartmann, L. 2001, ApJ, 550, 944

Naylor, T. 2009, MNRAS, 399, 432

Ostriker, E. C., \& Shu, F. H. 1995, ApJ, 447, 813

Pecaut, M. J., \& Mamajek, E. E. 2013, ApJS, 208, 9

Pecaut, M. J., \& Mamajek, E. E. 2016, MNRAS, 461, 794

Percy, J. R., Gryc, W. K., Wong, J. C.-Y., \& Herbst, W. 2006, PASP, 118, 1390

Press, W. H., Teukolsky, S. A., Vetterling, W. T., \& Flannery, B. P. 1992, Numerical recipes in C. The art of scientific computing, 2nd edn. (Cambridge: University Press)

Prisinzano, L., Damiani, F., Micela, G., \& Pillitteri, I. 2007, A\&A, 462, 123

Rebull, L. M. 2001, AJ, 121, 1676

Rebull, L. M., Makidon, R. B., Strom, S. E., et al. 2002, AJ, 123, 1528

Rebull, L. M., Stauffer, J. R., Megeath, S. T., Hora, J. L., \& Hartmann, L. 2006, ApJ, 646, 297

Reipurth, B., Yu, K. C., Moriarty-Schieven, G., et al. 2004, AJ, 127, 1069

Rhode, K. L., Herbst, W., \& Mathieu, R. D. 2001, AJ, 122, 3258

Rodríguez-Ledesma, M. V., Mundt, R., \& Eislöffel, J. 2009, A\&A, 502, 883

Romanova, M. M., Ustyugova, G. V., Koldoba, A. V., \& Lovelace, R. V. E. 2004, ApJ, 610, 920

SAS Institute Inc. 2012, SAS/STAT 12.1 User's Guide (Cary, NC: Author)

Scargle, J. D. 1982, ApJ, 263, 835

Shu, F., Najita, J., Ostriker, E., et al. 1994, ApJ, 429, 781

Siess, L., Dufour, E., \& Forestini, M. 2000, A\&A, 358, 593

Sousa, A. P., Alencar, S. H. P., Bouvier, J., et al. 2016, A\&A, 586, A47

Stassun, K. G., Mathieu, R. D., Mazeh, T., \& Vrba, F. J. 1999, AJ, 117, 2941

Stauffer, J., Cody, A. M., Baglin, A., et al. 2014, AJ, 147, 83

Stauffer, J., Cody, A. M., McGinnis, P., et al. 2015, AJ, 149, 130

Stauffer, J., Cody, A. M., Rebull, L., et al. 2016, AJ, 151, 60

Stetson, P. B. 1996, PASP, 108, 851

Sung, H., \& Bessell, M. S. 2010, AJ, 140, 2070

Sung, H., Bessell, M. S., Chun, M.-Y., Karimov, R., \& Ibrahimov, M. 2008, AJ, 135,441

Sung, H., Stauffer, J. R., \& Bessell, M. S. 2009, AJ, 138, 1116

Teixeira, P. S., Lada, C. J., Young, E. T., et al. 2006, ApJ, 636, L45

Tinker, J., Pinsonneault, M., \& Terndrup, D. 2002, ApJ, 564, 877

Ustyugova, G. V., Koldoba, A. V., Romanova, M. M., \& Lovelace, R. V. E. 2006, ApJ, 646, 304

Vasconcelos, M. J., \& Bouvier, J. 2015, A\&A, 578, A89

Venuti, L., Bouvier, J., Flaccomio, E., et al. 2014, A\&A, 570, A82

Venuti, L., Bouvier, J., Irwin, J., et al. 2015, A\&A, 581, A66

Vogel, S. N., \& Kuhi, L. V. 1981, ApJ, 245, 960

Weibull, W. 1951, J. Appl. Mech.-Trans. ASME, 18, 293

Wolf-Chase, G., Moriarty-Schieven, G., Fich, M., \& Barsony, M. 2003, MNRAS, 344, 809

Xiao, H. Y., Covey, K. R., Rebull, L., et al. 2012, ApJS, 202, 7

Zanni, C., \& Ferreira, J. 2013, A\&A, 550, A99 


\section{Appendix A: Classes of variables among NGC 2264 members}

Cody et al. (2014) provide a nice illustration of the diversity of light curve morphologies observed across the disk-bearing sample of NGC 2264 members. Here we extended their classification to the full set of members. A brief description of different morphology classes and of possible physical interpretations is presented in the following.

- Burster (B): light curve exhibiting sudden, rapid (0.11 day) rises in flux, followed by decreases on comparable timescales. Accretion instabilities appear to be driving the behavior displayed by this group of objects (Stauffer et al. 2014).

- Dipper (D): light curve characterized by transient optical fading events, possibly linked with extinction by circumstellar material. In some cases (e.g., AA Tau; Bouvier et al. 2007a), these events may recur periodically (likely resulting from warps located in the inner disk), although displaying changes in depth and/or shape (quasi-periodic dipper, QPD) from one cycle to the next.

- Spotted $(P)$ : flux variations dominated by surface spot modulation. Light curves may exhibit a definite, stable pattern over thousands of rotational cycles (Grankin et al. 2008; Venuti et al. 2015).

- Multi-periodic (MP): beating-like light curves or superposition of separate timescales of modulation. Several physical processes, such as differential rotation, spot evolution/migration, stellar pulsations may contribute to objects in this class.

- Eclipsing binary (EB): the light variation trend due to spot modulation and/or disk occultation is interspersed with periodic eclipses when one of the two stars in the binary system passes in front of the other during its orbital motion (e.g., Gillen et al. 2014).

- Stochastic (S): light curves exhibiting prominent flux changes on a variety of timescales, with no preference for fading or brightening events and no obvious periodicity. Time-dependent accretion onto the star, resulting in transient hot spots, may drive the observed variability for this class of objects (Stauffer et al. 2016).

- Long-timescale variable $(L)$ : variability for these objects grows or declines systematically up to the longest timescale of observation. These long timescales of variability may reflect disk dynamics beyond the inner edge.

\section{Appendix B: Impact of bin size and phase on the shape and features of the period histogram}

While in Fig. 5 and throughout the main paper text we use the commonly adopted bin size of $1 \mathrm{~d}$ and bin phase of 0 (i.e., bin scheme starting from $0.0 \mathrm{~d}$ ) for the period histogram, in this Appendix we explore how the histogram shape would be affected
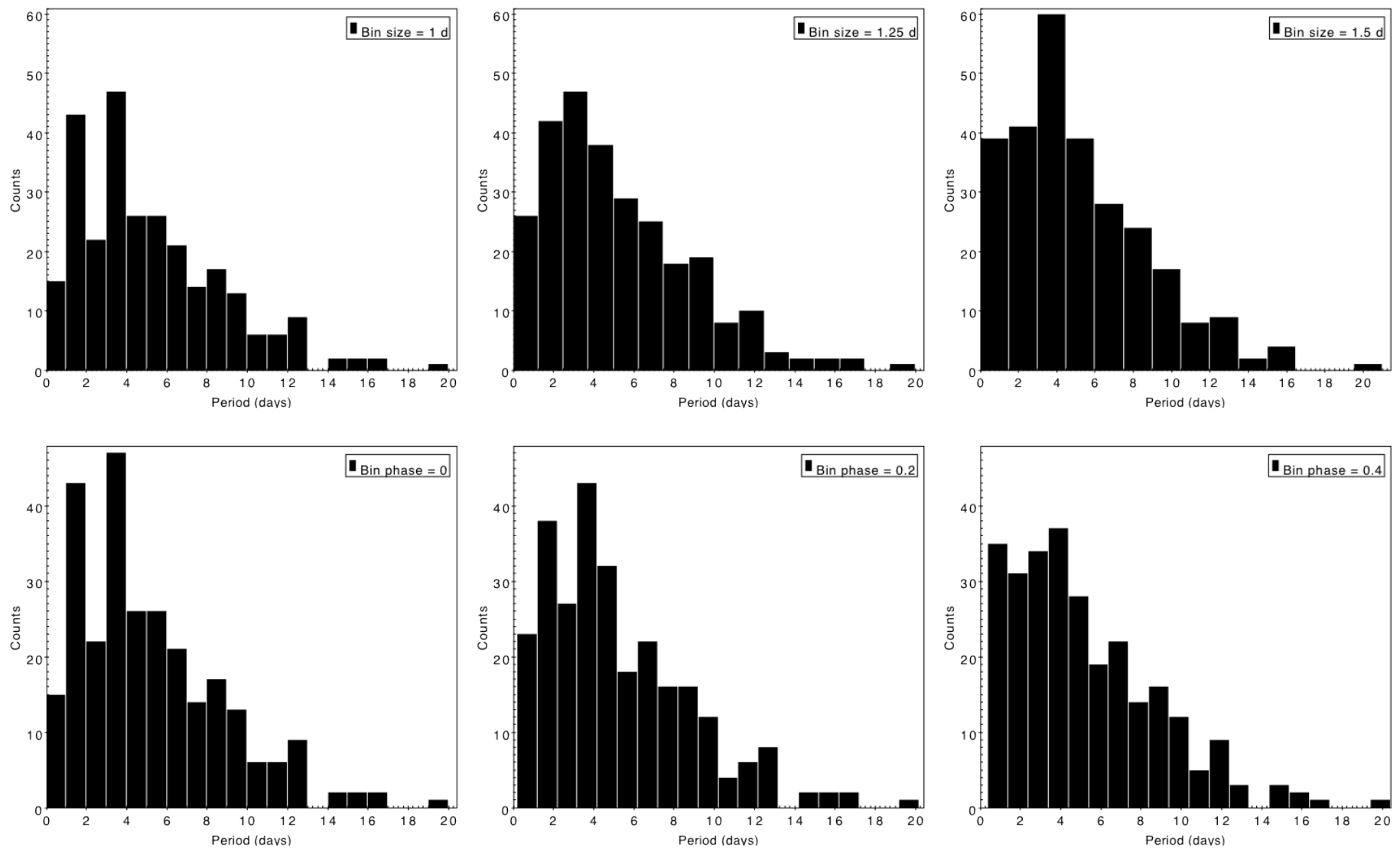

Fig. B.1. Illustration of how the shape and features of the period histogram in Fig. 5 would evolve when varying the bin size (upper panels) or the bin phase (lower panels). The starting bin size and phase (of $1 \mathrm{~d}$ and 0 , respectively; these are shown on the upper left panel and on the lower left panel of this figure) correspond to those adopted in Fig. 5 and considered for the subsequent analysis. In the upper panel, the bin size is progressively increased by $0.25 \mathrm{~d}$ at each step from the left diagram to the right diagram; in the lower panel, a fixed bin size of $1 \mathrm{~d}$ is adopted and the bin center is progressively shifted by $0.2 \mathrm{~d}$ at each step from the left diagram to the right diagram. 

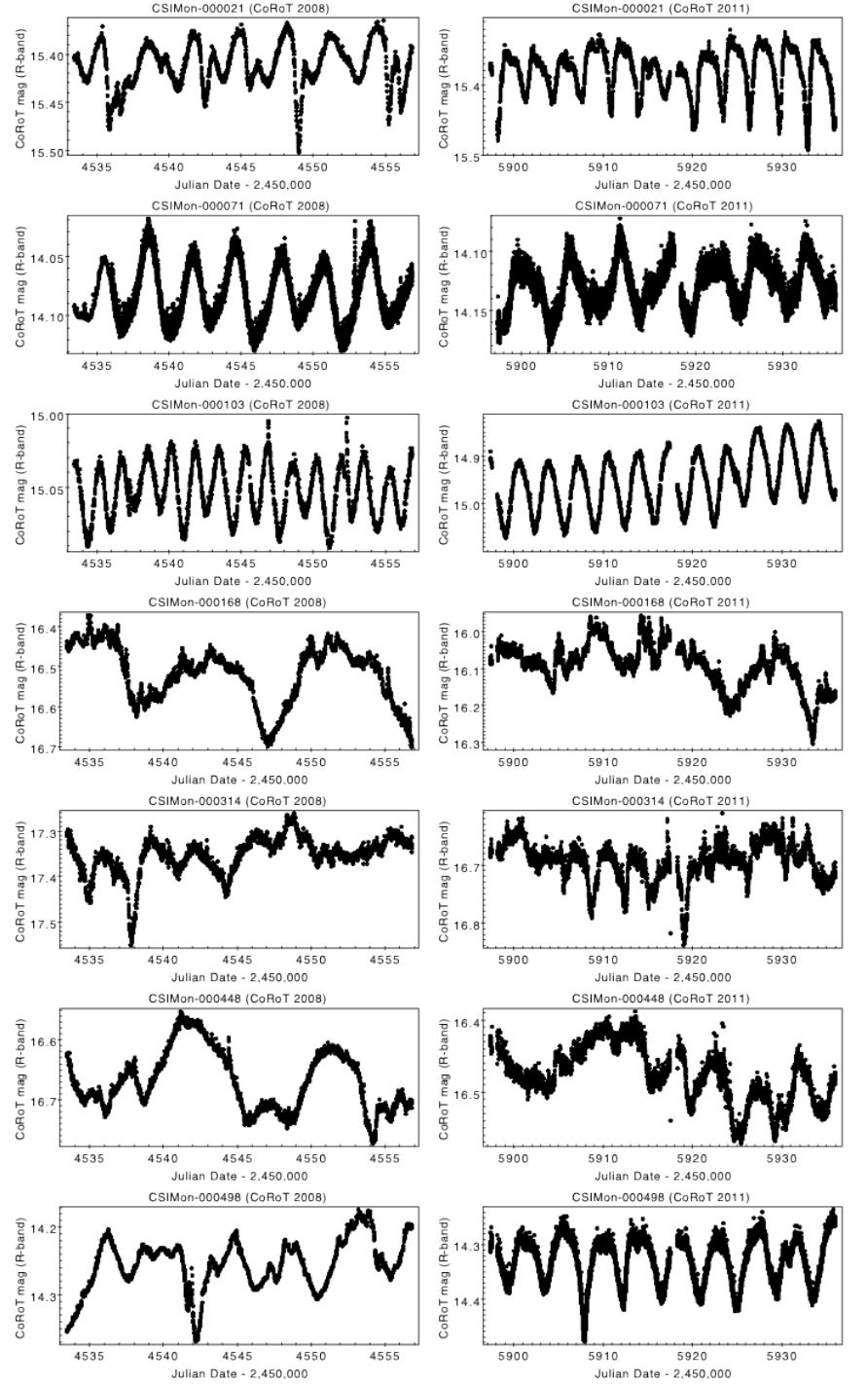
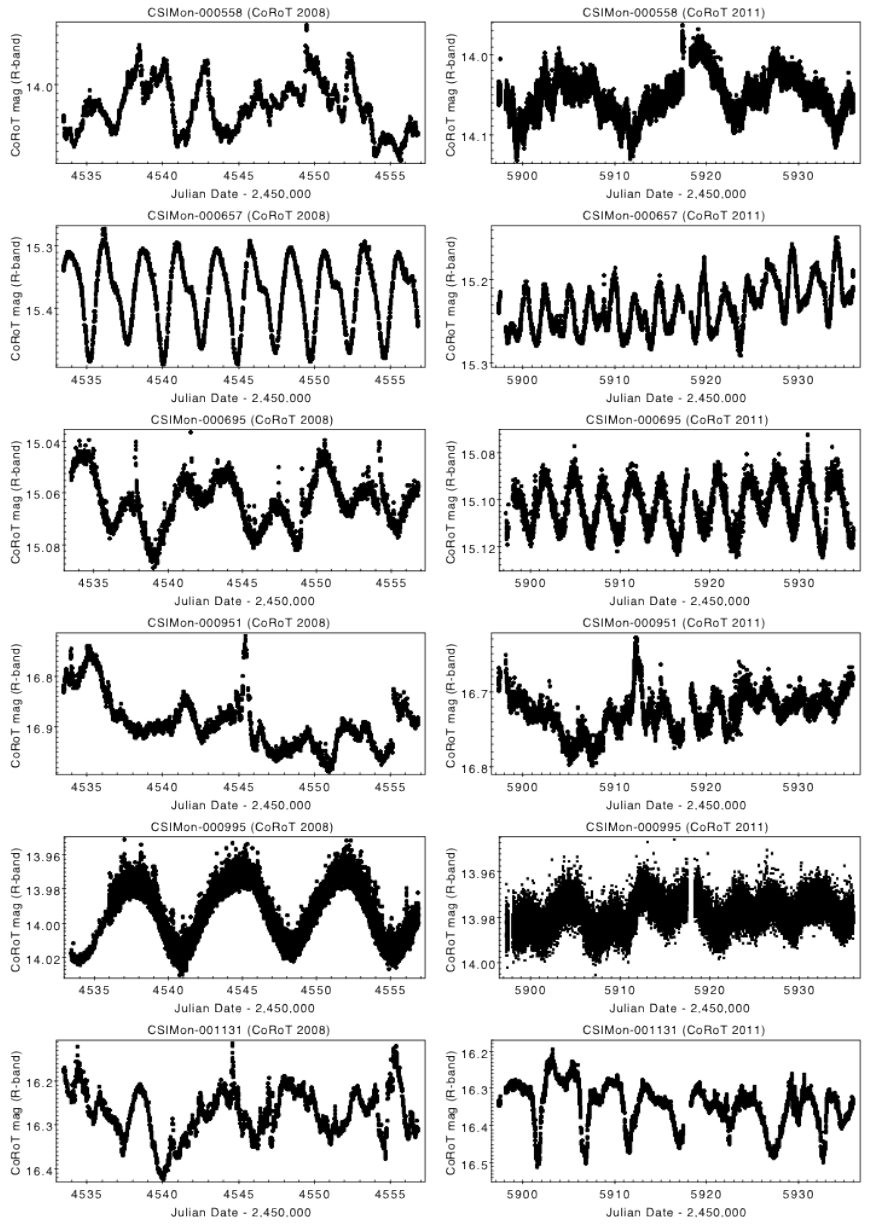

Fig. C.1. Comparison of the light curves obtained with CoRoT in 2008 (left) and 2011 (right) for objects with discrepant period estimates between Affer et al. (2013) and this study.

by different choices in bin size and phase. This is illustrated in Fig. B.1.

The upper panel of Fig. B.1 shows how the observed histogram shape and features evolve when varying the bin size. A smaller bin size of $0.5 \mathrm{~d}$ (not shown here) would produce an histogram with the same broad features and better resolved peaks than that shown in Fig. 5 and reported in the upper left diagram of Fig. B.1. Conversely, increasing the bin size would determine the peak at shorter periods to progressively merge with the peak at longer periods; this trend can be seen when reading the upper panel of Fig. B.1 from the left to the right.

Similarly, the lower panel of Fig. B.1 shows the change in shape of the period histogram when shifting the bin centers along the period axis. Small shifts (left and middle panel in the lower part of Fig. B.1) do not have a strong impact on the global properties of the period histogram: hints of two peaks can still be observed when we adopt a bin phase of 0.2. However, as discussed in Sect.4.1, larger shifts, comparable to the width of a single peak, would determine these to redistribute into two neighboring histogram channels, hence transforming the distribution into a flatter distribution with no significant peaks between 0 and $5 \mathrm{~d}$ (lower right panel of Fig. B.1).

\section{Appendix C: Discrepant period estimates between this work and Affer et al. (2013)}

As shown in Fig. 4, in a small number of cases the period estimates derived in the present study are in contrast with previous period estimates reported in the literature. In this Appendix, we illustrate and discuss the objects individually that are common to our sample and Affer et al.'s (2013) sample, that are outliers with respect to the equality line on the left panel of Fig. 4. A detailed comparison of the light curves on which the period analysis was performed in the two studies is shown in Fig. C.1. For comparison purposes, in some of the cases examined in this and in the next Appendix we will also refer to the preliminary results of a similar analysis of rotation that is being conducted on the mid-IR time series photometry obtained with Spitzer/IRAC during the CSI 2264 campaign (Rebull et al., in prep.). The relevant light curves at $3.6 \mu \mathrm{m}$ are shown in Fig. C.2.

CSIMon-000021. The period reported in Affer et al. (2013) is about twice that reported in the present study. This object is classified as a narrow dipper in Stauffer et al. (2015), and fading events recur in a regular pattern at a period of $3.15 \mathrm{~d}$. The 

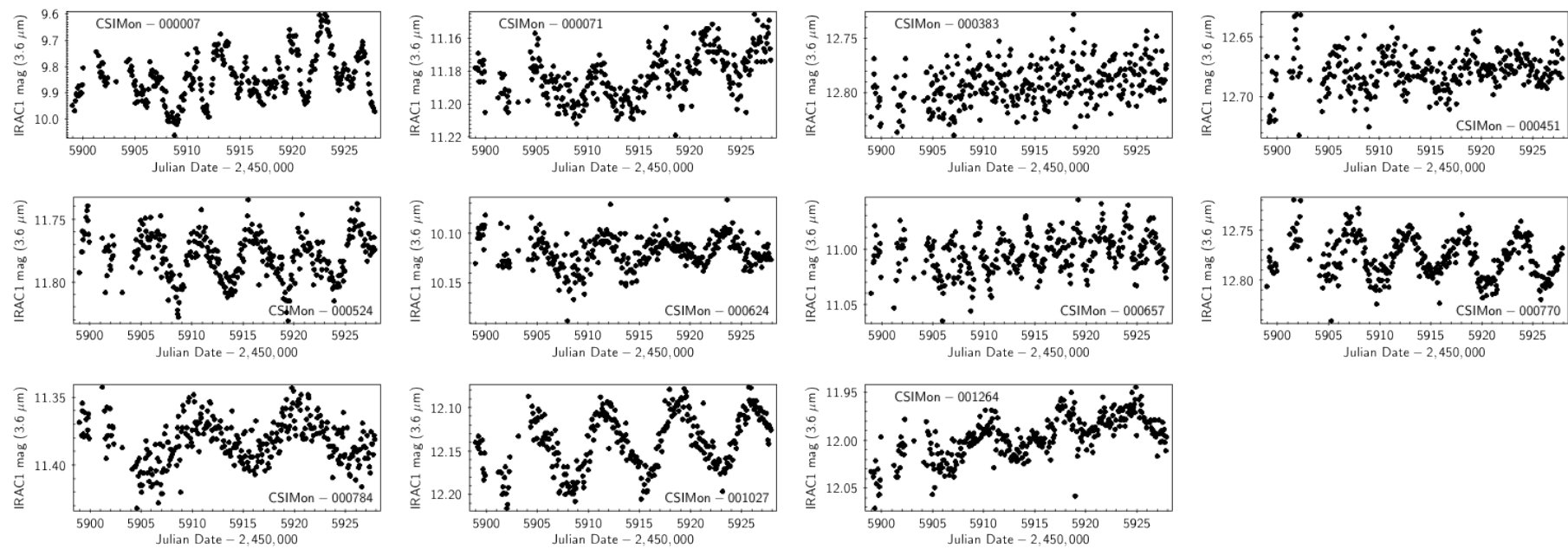

Fig. C.2. Mid-IR light curves, obtained with the Spitzer/IRAC $3.6 \mu \mathrm{m}$ channel, for some of the sources discussed in Appendices C and D. A detailed description of Spitzer/IRAC data obtained within the CSI 2264 campaign is presented in Cody et al. (2014).

CoRoT light curve obtained in 2008 instead appears as a superposition of a spot modulation component, which has a visual period close to the value measured here, and of a dip component, which seems to occur at a longer periodicity of about twice the spot modulation periodicity.

CSIMon-000071. The period reported in Affer et al. (2013) is about half that found in the present study. The light curve "unit" from the CoRoT run of 2008 appears to be M-shaped, with the secondary minimum only slightly less deep than the first minimum. Conversely, the 2011 light curve has a single minimum and a period of $5.41 \mathrm{~d}$, consistent with the preliminary results obtained from the period analysis of CSI 2264 Spitzer/IRAC light curves (Rebull et al., in prep.).

CSIMon-000103. The period reported here is about twice the period in Affer et al. (2013), but is consistent with the period estimate reported in Lamm et al. (2004). Similar to the case of CSIMon-000071, the CoRoT light curve observed in 2008 for this object had two minima in a period unit; as illustrated in Fig. 2, a periodogram-based analysis of such cases may incur in an erroneous period detection at the half period.

CSIMon-000168. This is a long-period object, with a period estimate of $10.02 \mathrm{~d}$ from this study and of $8.61 \mathrm{~d}$ from Affer et al. (2013). The two period estimates are only marginally inconsistent when we consider the associated uncertainty from Eq. (4); note that no error estimate is reported in Affer et al. (2013). As shown in Fig. C.1, at both epochs the light curve is not entirely smooth.

CSIMon-000314. The period reported in Affer et al. (2013) is about twice the period reported here. As illustrated in Fig. C.1, the light curve of this object is not very well behaved at any epochs. No other period estimates are available for this object from other datasets in the CSI 2264 campaign or from previous studies in the literature.
CSIMon-000448. The period reported in Affer et al. (2013) is about twice the period reported here. As in the case on CSIMon000314, the light curve is not entirely smooth.

CSIMon-000498. The period reported in Affer et al. (2013) is about twice the period reported here. As shown in Fig. C.1, the flux modulation appears to be more regular at the 2011 epoch than at the 2008 epoch. Lamm et al. (2004) also found a period value close to the one reported here.

CSIMon-000558. Case similar to CSIMon-000168: long-period object, with measured period of $11.71 \mathrm{~d}$ in this study and of $10.17 \mathrm{~d}$ in Affer et al. (2013). The two estimates are only marginally inconsistent within the error estimated on our derivation of period.

CSIMon-000657. The period reported in Affer et al. (2013) is about half the period reported here. Lamm et al. (2004) report a period estimate consistent with that of Affer et al. (2013), and preliminary results from the period analysis of Spitzer/IRAC light curves appear to agree with these. Figure C. 1 shows that the 2008 CoRoT light curve may actually consist of two separate, alternating features of slightly different shape; this would imply that the periodogram peak indicated in Affer et al. (2013) corresponds to half the actual rotation rate.

CSIMon-000695. The period reported in Affer et al. (2013) is about thrice that found here. Figure C. 1 shows that a modulation is well seen in the CoRoT 2011 light curve, whereas the pattern is more fragmented on the 2008 light curve.

CSIMon-000951. The period reported in Affer et al. (2013) is significantly larger than that found in this study. As can be observed in Fig. C.1, the light curve is partly irregular at any epochs. We note that the period estimate reported in Lamm et al. (2004) is consistent with the one that we report here.

CSIMon-000995. Another object with long periodicity; Affer et al. (2013) report $P=7.02 \mathrm{~d}$, while we derive here 

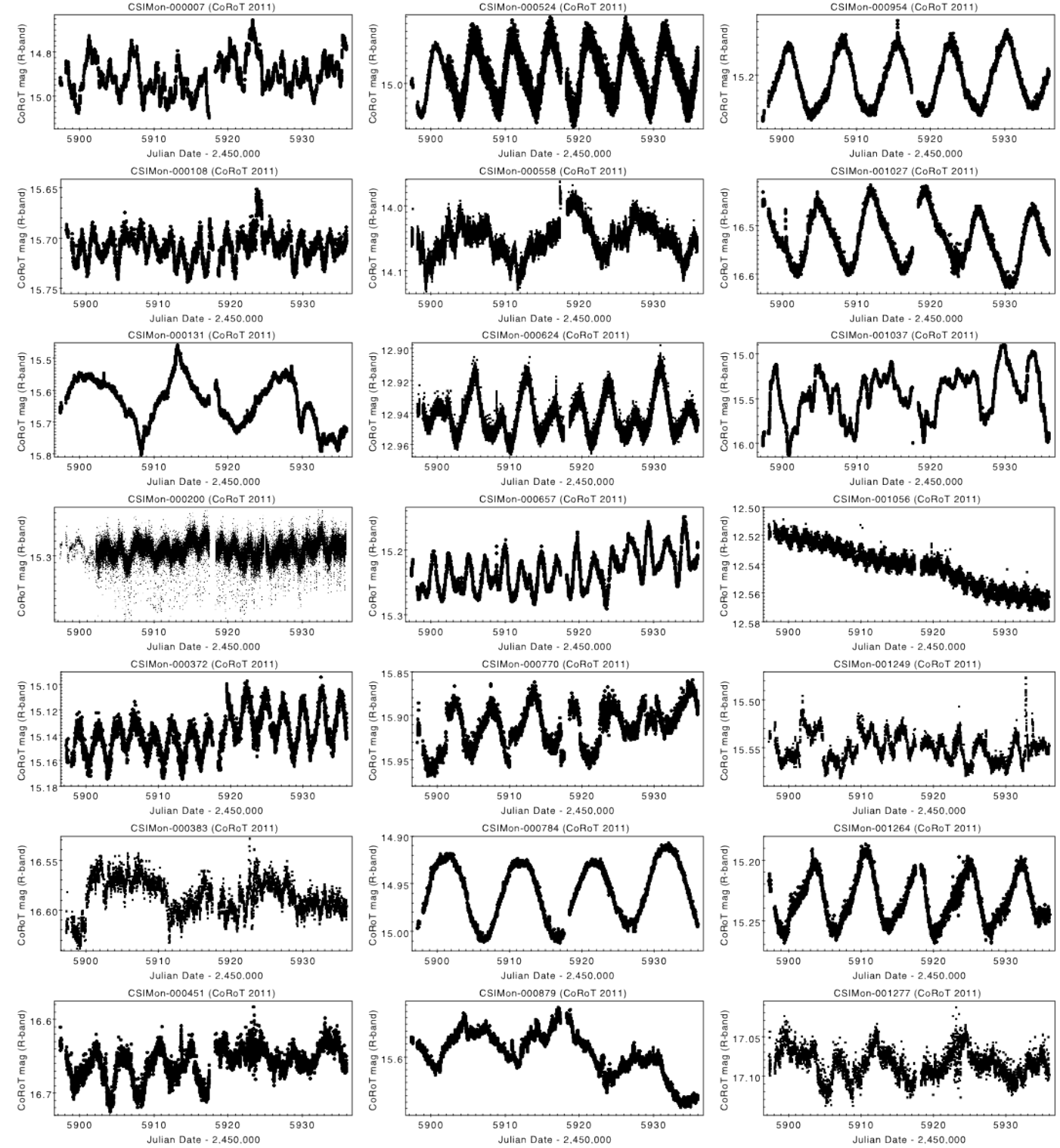

Fig. D.1. CoRoT 2011 light curves for objects whose period estimate derived in this study are in disagreement with the periods reported in Cieza \& Baliber (2007).

$P=9.28$ d. The light curve comparison shown in Fig. C.1 illustrates that the modulated pattern was better traced in 2008 .

CSIMon-001131. The period reported in Affer et al. (2013) is about twice the period we report here. The light curve comparison shown in Fig. C.1 illustrates that the flux variations were better behaved in 2011 .

\section{Appendix D: Discrepant period estimates between this work and Cieza \& Baliber (2007, after Lamm et al. 2004 and Makidon et al. 2004)}

Figure D.1 illustrates the CoRoT light curves analyzed in this study for objects whose derived period is in disagreement with the estimate reported in Cieza \& Baliber (2007). In the following, these discrepant cases are discussed individually. For some of them, we also discuss indications of periodicity deduced from CSI 2264 Spitzer/IRAC photometry; the relevant light curves at $3.6 \mu \mathrm{m}$ are shown in Fig. C.2.
CSIMon-000007. The period reported in Cieza \& Baliber (2007) for this object is close to $1 \mathrm{~d}(0.93 \mathrm{~d})$, whereas it has a period of $3.19 \mathrm{~d}$ in our study (see Table 4). The light curve profile is partly irregular (this is one of the objects classified as dominated by stochastic accretion bursts by Stauffer et al. 2014); preliminary results from the analysis of the Spitzer/IRAC dataset (Rebull et al., in prep.) would also suggest a periodicity of $3.17 \mathrm{~d}$.

CSIMon-000108. The period reported in Cieza \& Baliber (2007) for this object is of $1.37 \mathrm{~d}$, whereas it has a period of $4.06 \mathrm{~d}$ in this study. The light curve unit appears to have two maxima; indication for the period reported in this study derives primarily from the ACF and SL methods.

CSIMon-000131. The period reported in Cieza \& Baliber (2007) is about half that found in this study (6.4 d and $12.87 \mathrm{~d}$, respectively). A long-period modulation can be seen in the CoRoT 2011 light curve, although it has an irregular variability component superimposed. 
CSIMon-000200. The period reported in Cieza \& Baliber (2007) is about twice that found in this study (3.88 d and $1.92 \mathrm{~d}$, respectively). The light curve exhibits a fairly small amplitude of variability, but a modulation effect is clearly seen in the CoRoT light curve. The light curve unit might be M-shaped (with two maxima), but that is not entirely evident from the light variation pattern and the derived period diagrams; for this reason, we opted here for the shorter period.

CSIMon-000372. The period reported in Cieza \& Baliber (2007) is about half that found in this study (1.3 d and $2.57 \mathrm{~d}$, respectively). A modulation at a period of about $2.5 \mathrm{~d}$ is clearly seen on the CoRoT 2011 light curve shown in Fig. D.1, and this result is supported by the analysis of the former CoRoT dataset by Affer et al. (2013).

CSIMon-000383. The period reported in Cieza \& Baliber (2007) is about half that found in this study $(0.51 \mathrm{~d}$ and $1.03 \mathrm{~d}$, respectively). The CoRoT 2011 light curve for this object is affected by instrument systematics, which may impact the results of the period analysis. Preliminary results of the analysis of Spitzer/IRAC light curves (Rebull et al., in prep.) seem to support the period value reported in Cieza \& Baliber (2007), after Makidon et al. (2004).

CSIMon-000451. The period reported in Cieza \& Baliber (2007) for this object is $0.68 \mathrm{~d}$, whereas it has a period of $4.52 \mathrm{~d}$ in our study (see Table 4). A modulation effect of several days is well observed during the first half of the CoRoT 2011 light curve, although it becomes more irregular during the second fraction of the monitored time span. A close periodicity to the one we report here is suggested by preliminary results of the Spitzer/IRAC light curve analysis (Rebull et al., in prep.).

CSIMon-000524. The period reported in Cieza \& Baliber (2007) for this object is $1.23 \mathrm{~d}$, whereas a period of $5.15 \mathrm{~d}$ is found here. The periodic pattern is clearly outlined in the CoRoT light curve, and the period found here is supported by the Spitzer/IRAC light curves (Rebull et al., in prep.)

CSIMon-000558. The period reported in Cieza \& Baliber (2007) for this object is close to $1 \mathrm{~d}(0.88 \mathrm{~d})$, whereas it has a period of $11.71 \mathrm{~d}$ in our study (see Table 4). A long-term modulation can be clearly observed on the CoRoT 2011 light curve, and is supported by Affer et al.'s (2013) results. No significant evidence of shorter periodicities results from our analysis.

CSIMon-000624. The period reported in Cieza \& Baliber (2007) is about half that found in this study (3.73 d and $7.47 \mathrm{~d}$, respectively). The CoRoT 2011 light curve appears to be the alternation of taller and shorter maxima; the indication for the period reported here derives from the ACF and SL methods, whereas the periodogram peaks at half that value (as discussed for the case in Fig. 2). A similar period to the one reported here is suggested by the preliminary results of the Spitzer/IRAC light curve analysis (Rebull et al., in prep.).

CSIMon-000657. The period reported in Cieza \& Baliber (2007) is about half that found in this study $(2.43 \mathrm{~d}$ and $4.83 \mathrm{~d}$, respectively). Affer et al. (2013) also report a period consistent with that listed in Cieza \& Baliber (2007; see discussion about this object in Appendix C). The light curve unit in the CoRoT 2011 dataset appears to have a complex and time-varying shape, with several maxima. The value of period reported here is based on the ACF and SL diagnostic tools; the periodogram peaks at half its value.

CSIMon-000770. The period reported in Cieza \& Baliber (2007) for this object is close to $1 \mathrm{~d}(0.84 \mathrm{~d})$, whereas it has a period of $5.44 \mathrm{~d}$ in our study (see Table 4 ). The same value of period that we find here is suggested by preliminary Spitzer/IRAC results (Rebull et al., in prep.), and this was also reported in Affer et al. (2013) from the analysis of the previous CoRoT run on NGC 2264.

CSIMon-000784. The period reported in Cieza \& Baliber (2007) for this object is close to $1 \mathrm{~d}(0.91 \mathrm{~d})$, whereas it has a period of $10.10 \mathrm{~d}$ in our study (see Table 4). The modulation is clearly seen in the CoRoT 2011 light curve (see Fig. D.1; the same value of period is suggested by Spitzer/IRAC data.

CSIMon-000879. The period reported in Cieza \& Baliber (2007) for this object is close to $1 \mathrm{~d}(0.91 \mathrm{~d})$, whereas it has a period of $11.40 \mathrm{~d}$ in our study (see Table 4 ). The light curve for this object is not very regular, but a long-period modulation can be detected fairly clearly by eye (see Fig. D.1).

CSIMon-000954. The period reported in Cieza \& Baliber (2007) for this object is close to $1 \mathrm{~d}(0.88 \mathrm{~d})$, whereas it has a period of $7.35 \mathrm{~d}$ in our study (see Table 4 ). The modulation pattern with a periodicity of several days is very clearly seen in the CoRoT 2011 light curve.

CSIMon-001027. The period reported in Cieza \& Baliber (2007) for this object is close to $1 \mathrm{~d}(1.15 \mathrm{~d})$, whereas it has a period of $7.22 \mathrm{~d}$ in our study (see Table 4). As in the previous case, the modulation pattern with a periodicity of several days is very clearly seen in the CoRoT 2011 light curve. A very similar result is derived from the analysis of Spitzer/IRAC light curves (Rebull et al., in prep.).

CSIMon-001037. The period reported in Cieza \& Baliber (2007) for this object is $12.09 \mathrm{~d}$, whereas a period of $8.88 \mathrm{~d}$ is found here. The actual light curve pattern is fairly irregular, which may affect the accuracy of the derived period for this object, although a global effect of modulation with long periodicity can be detected. No periodicity was detected for this object in the study of Affer et al. (2013).

CSIMon-001056. The period reported in Cieza \& Baliber (2007) is about half that found in this study $(0.78 \mathrm{~d}$ and $1.52 \mathrm{~d}$, respectively). The CoRoT 2011 light curve has a small amplitude, superimposed over a spurious long-term trend, but the modulated pattern can be clearly seen.

CSIMon-001249. The period reported in Cieza \& Baliber (2007) is about twice that found in this study (3.87 d and $1.95 \mathrm{~d}$, respectively). The CoRoT 2011 light curve is partly affected by instrument systematics. 

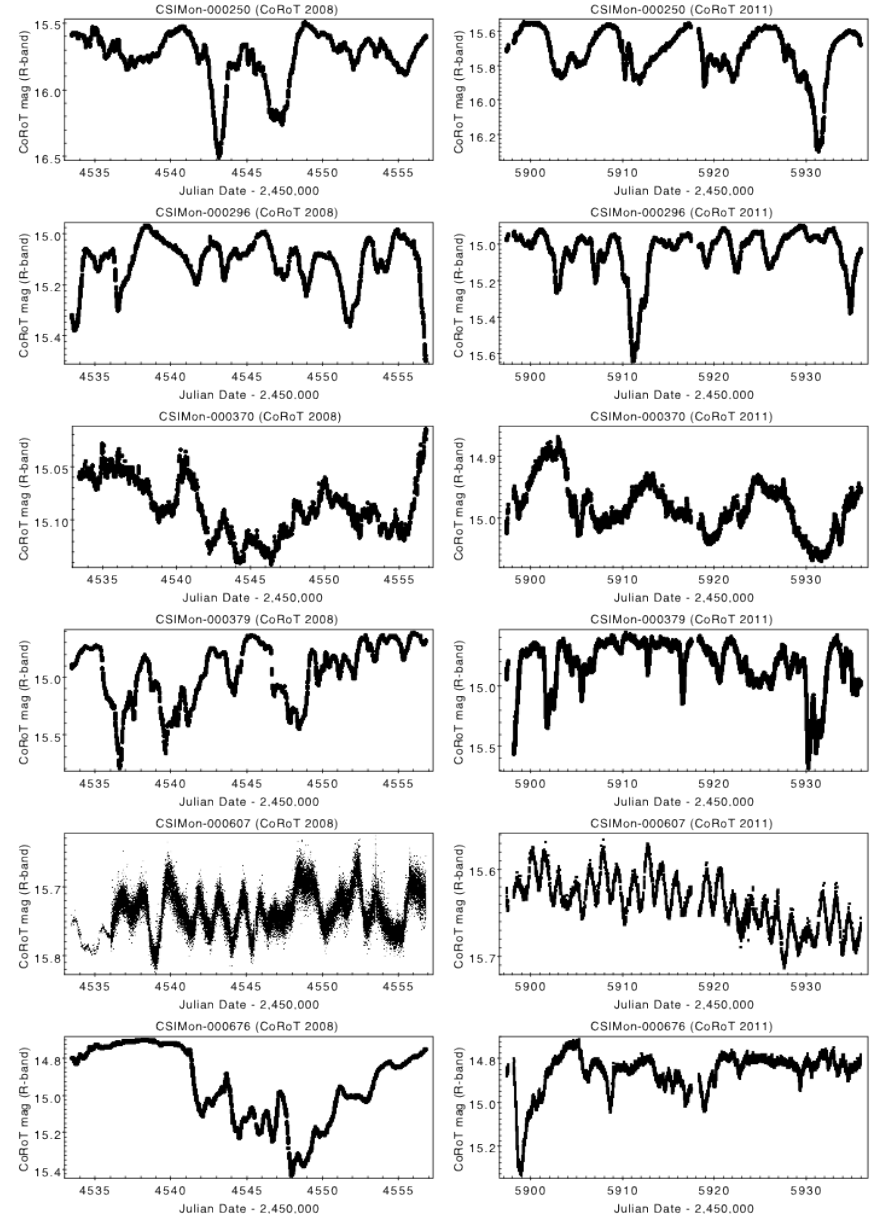
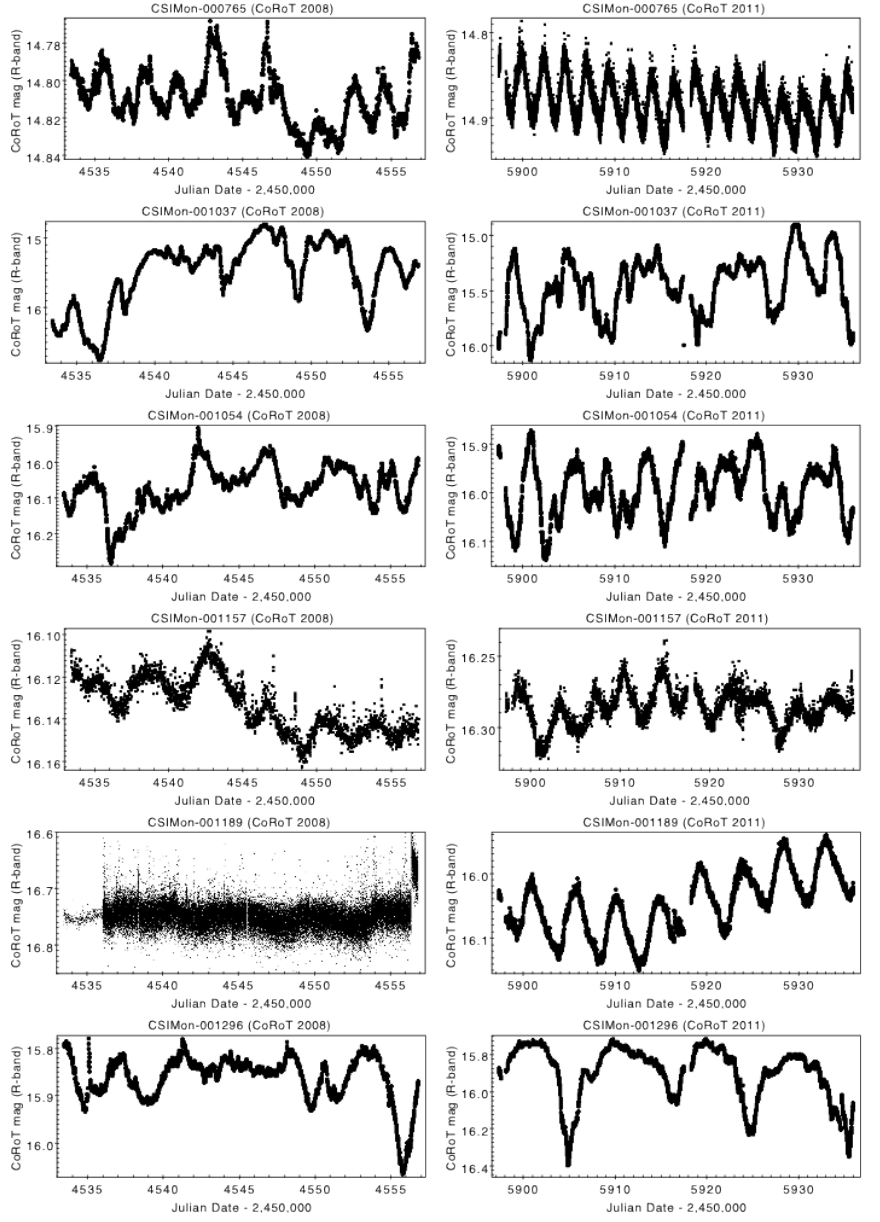

Fig. E.1. Twelve cases of objects (six on the left side, six on the right side of the picture) that had aperiodic CoRoT light curves in 2008 (periodicity data from Affer et al. 2013) but are assigned a periodicity here based on their 2011 CoRoT light curves. Each object corresponds to a pair of panels: the first illustrates the CoRoT 2008 light curve; the second illustrates the CoRoT 2011 light curve. Cases shown are, from top to bottom: CSIMon250, 296, 370, 379, 607, 676 (left side); CSIMon-765, 1037, 1054, 1157, 1189, 1296 (right side).

CSIMon-001264. The period reported in Cieza \& Baliber (2007) for this object is close to $1 \mathrm{~d}(0.88 \mathrm{~d})$, whereas it has a period of $7.17 \mathrm{~d}$ in our study (see Table 4). A modulated pattern with a periodicity of several days is clearly seen for this object on the CoRoT 2011 light curve. A close periodicity to the one we report here was found by Affer et al. (2013), and it also appears from the preliminary results from Spitzer/IRAC light curves (Rebull et al., in prep.).

CSIMon-001277. The period reported in Cieza \& Baliber (2007) for this object is $5.29 \mathrm{~d}$, whereas a period of $12.21 \mathrm{~d}$ is found here. The light curve in the CoRoT 2011 dataset is not very well behaved, which may impact our period determination for this object.

\section{Appendix E: Objects which appear to have evolved from periodic to aperiodic or vice versa between the two CoRoT runs}

About $5 \%$ of objects in our sample (Table 4), found to be periodic here, were reported as non-periodic in the study of Affer et al. (2013). Similarly, about 5\% of objects in our sample, classified as non-periodic in Table 4 , had a periodicity assigned in Affer et al. (2013).
Some of these cases can be attributed to the fact that their light curves exhibit a certain degree of irregularity, or that the observed flux variations develop on fairly long timescales ( $\sim$ weeks); these situations translate to more unclear period assessment. In other cases, the light curves at one or the other epoch are affected by systematics, that hampers the period analysis. For a fraction of cases, however, the discrepant result on the light curve periodicity/non-periodicity at the two epochs reflects a real evolution in photometric behavior between the two epochs.

Figure E.1 illustrates twelve cases of objects that exhibited aperiodic light curves in 2008 but have a period measured from the 2011 epoch and reported in Table 4. We can identify two main groups among the examples shown. The first (e.g., the CTTS CSIMon-000370 and CSIMon-000765, respectively third panel on the left and first panel on the right side of the picture, from the top) consists of objects with nicely modulated light curves at one epoch and more irregular light curve shapes at the other epoch. These light curve changes may be driven by a variation in the accretion activity of the objects: more intense in the first epoch, with light curves dominated by a changing mix of cold magnetic spots and hot accretion spots at the stellar surface (e.g., Herbst et al. 1994); more moderate at the second epoch, when well-behaved cold spot modulation prevails in the light curve. The second group of cases is exemplified by objects CSIMon-000296 and CSIMon-001296 (CTTS; second panel on 

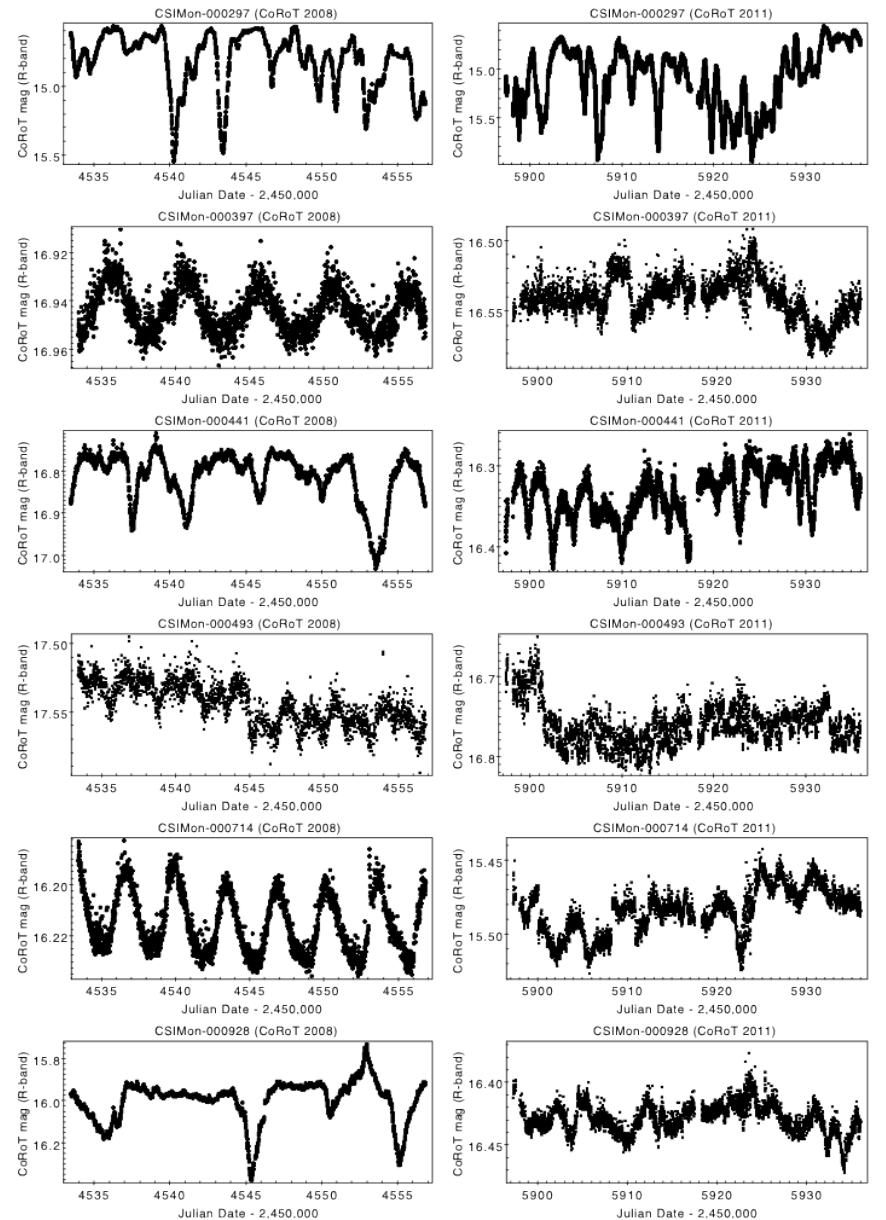
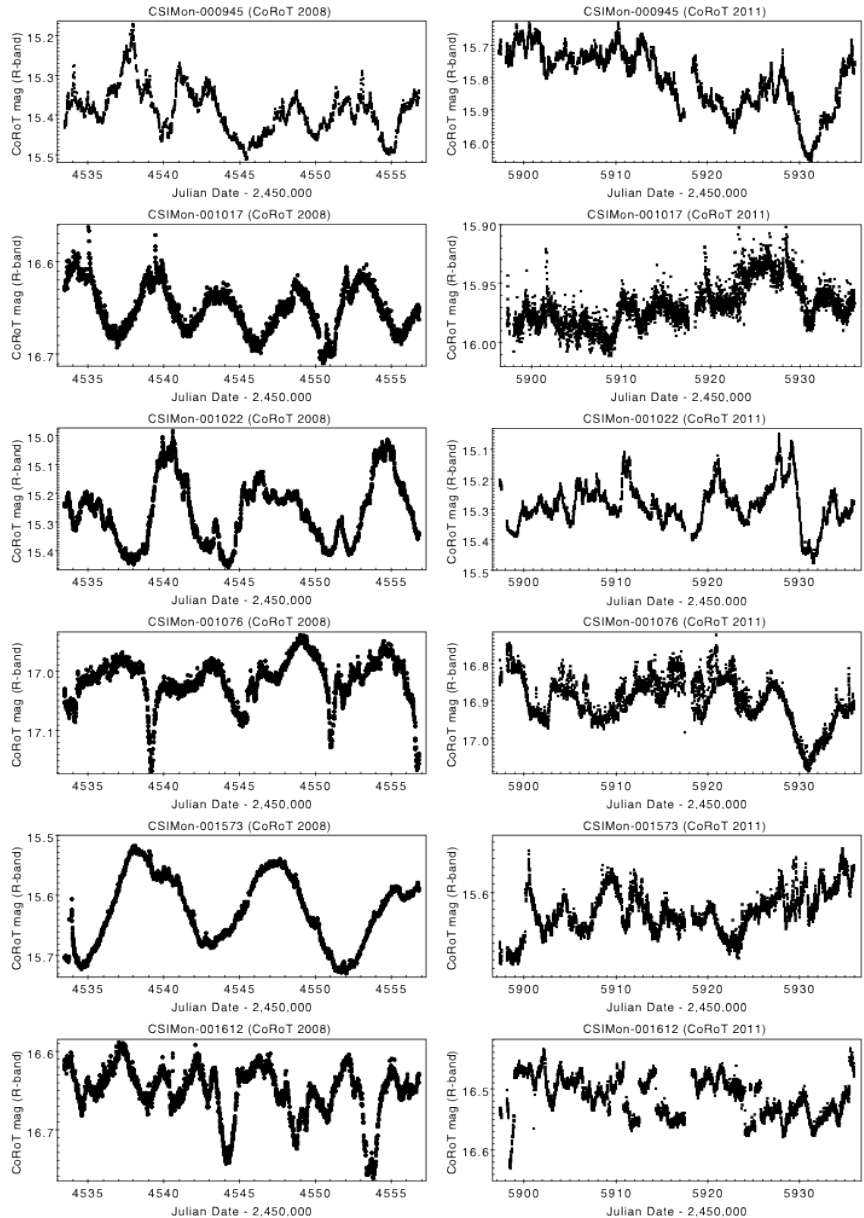

Fig. E.2. Twelve cases of objects (six on the left side, six on the right side of the picture) that had periodic CoRoT light curves in 2008 (periodicity data from Affer et al. 2013) but are classified as aperiodic here based on their 2011 CoRoT light curves. Each object corresponds to a pair of panels: the first illustrates the CoRoT 2008 light curve; the second illustrates the CoRoT 2011 light curve. Cases shown are, from top to bottom: CSIMon-297, 397, 441, 493, 714, 928 (left side); CSIMon-945, 1017, 1022, 1076, 1573, 1612 (right side).

the left and last panel on the right side of the picture, from the top); these exhibit a dipper-like light curve (flat luminosity maximum interspersed by flux dips associated with extinction events from circumstellar material; McGinnis et al. 2015). The extinction events, possibly linked to inner disk warps at the base of the accretion funnels, occur aperiodically at one epoch and periodically at the other; as discussed in McGinnis et al. (2015), this may be due to a transition between unstable and stable accretion regimes. Finally, special mention goes to the object CSIMon001189 (fifth panel from the top on the right side of Fig. E.1, a WTTS whose nearly flat-band light curve observed in 2008 (uniform spot distribution + instrumental noise?) evolved into a smooth modulated pattern recorded in 2011.
Similarly, Fig. E.2 illustrates twelve cases of objects with detected periodicity in Affer et al. (2013) from 2008 light curves, that appear to be aperiodic in the 2011 epoch. Again, we can identify the two main types of photometric behaviors and evolution discussed in the previous paragraph. In some cases, e.g., the CTTS CSIMon-001573 (fifth panel from the top on the right side of the picture), the predominantly modulated light curve pattern observed in 2008 evolved into a more irregular, possibly hot-spot dominated flux variation trend in 2011. In other cases, e.g., the CTTS CSIMon-000928 (last panel from the top on the right side of the figure), the periodic, AA Tau-like dipper profile observed at the 2008 epoch evolved into an aperiodic light curve trend in 2011. 


\section{Appendix F: Additional table}

Table F.1. Periodicity of CoRoT light curves for NGC 2264 members.

\begin{tabular}{|c|c|c|c|c|c|c|c|c|c|c|}
\hline CSIMon-\# & $\mathrm{RA}^{1}$ & $\operatorname{Dec}^{1}$ & Class $^{2}$ & CoRoT_2011 & $\mathrm{LC}^{3}$ & $M_{\star}\left(M_{\odot}\right)$ & $P_{2011}^{\mathrm{CoRoT}}(\mathrm{d})$ & $\mathrm{FAP}^{4}$ & $\mathrm{Q}^{5}$ & $P_{2008}^{\mathrm{CoRoT}}(\mathrm{d})^{6}$ \\
\hline 000005 & 100.32145 & 9.89435 & $\mathrm{w}$ & 616849481 & QPS & 0.31 & 3.998 & $5.0 \mathrm{E}-3$ & 1.13 & - \\
\hline 000006 & 100.52982 & 9.89571 & & 223998980 & $\mathrm{~N}$ & & & & & \\
\hline $000007^{*}$ & 100.47095 & 9.96739 & $\mathrm{c}$ & 223994721 & B & 0.69 & 3.192 & $<1 \mathrm{E}-4$ & 0.75 & \\
\hline 000008 & 100.45248 & 9.90322 & $\mathrm{w}$ & 616826337 & & 0.80 & & & & - \\
\hline 000009 & 100.53812 & 9.80132 & $\mathrm{w}$ & 223999591 & $\mathrm{U}$ & 1.30 & & & & \\
\hline 000011 & 100.32187 & 9.90900 & $\mathrm{c}$ & 223985009 & $\mathrm{~S}$ & 0.70 & & & & \\
\hline 000012 & 100.28892 & 9.93559 & & 602099712 & QPS & 1.13 & 2.961 & $<1 \mathrm{E}-4$ & 0.20 & - \\
\hline 000014 & 100.52775 & 9.69215 & $\mathrm{w}$ & 602087973 & $\mathrm{U}$ & 0.66 & & & & - \\
\hline 000015 & 100.53798 & 9.98410 & $\mathrm{w}$ & 223999581 & $\mathrm{~N}$ & 0.65 & & & & \\
\hline 000017 & 100.38329 & 10.00677 & $\mathrm{c}$ & 223988827 & $P$ & 1.13 & 4.771 & $<1 \mathrm{E}-4$ & -0.03 & 4.767 \\
\hline $000018^{\#,}$ & 100.30510 & 9.91908 & $\mathrm{w}$ & 223983925 & $\mathrm{Be}$ ? & 1.47 & 4.26 & $<1 \mathrm{E}-4$ & & 3.704 \\
\hline 000020 & 100.53847 & 9.73428 & $\mathrm{w}$ & 602091922 & QPS & 0.71 & 5.179 & $<1 \mathrm{E}-4$ & 0.27 & - \\
\hline 000021 & 100.24771 & 9.99595 & $\mathrm{c}$ & 223980412 & QPD & 1.20 & 3.147 & $<1 \mathrm{E}-4$ & 0.15 & 6.39 \\
\hline 000022 & 100.26904 & 10.01185 & $\mathrm{w}$ & 603809295 & $\mathrm{U}$ & 0.24 & & & & - \\
\hline 000024 & 100.48687 & 9.79589 & $\mathrm{w}$ & 603414392 & $\mathrm{~N}$ & 0.44 & & & & - \\
\hline 000029 & 100.26367 & 9.96528 & $\mathrm{w}$ & 223981349 & QPS & 0.70 & 8.012 & $<1 \mathrm{E}-4$ & 0.13 & 8.014 \\
\hline 000033 & 100.28027 & 9.97533 & $\mathrm{w}$ & 223982407 & $\mathrm{P}$ & 1.20 & 2.586 & $<1 \mathrm{E}-4$ & 0.09 & 2.582 \\
\hline 000035 & 100.44896 & 9.86731 & $\mathrm{w}$ & 223993180 & $\mathrm{P}$ & 1.69 & 2.413 & $<1 \mathrm{E}-4$ & 0.56 & 2.411 \\
\hline 000038 & 100.29532 & 10.01137 & w & 223983310 & QPS & 1.40 & 3.615 & $<1 \mathrm{E}-4$ & -0.55 & 3.589 \\
\hline 000045 & 100.47106 & 10.00069 & w & 616803281 & QP & 0.28 & 0.85 & $5.0 \mathrm{E}-4$ & - & - \\
\hline 000047 & 100.38365 & 10.01796 & $\mathrm{w}$ & 223988855 & $\mathrm{P}$ & & 1.342 & $<1 \mathrm{E}-4$ & 0.16 & - \\
\hline 000048 & 100.46442 & 9.89518 & & 223994268 & QPS & 1.40 & 3.631 & $<1 \mathrm{E}-4$ & 0.47 & 3.762 \\
\hline 000050 & 100.25639 & 10.01014 & $\mathrm{w}$ & 223980944 & QPS & 1.22 & 3.554 & $<1 \mathrm{E}-4$ & 0.10 & - \\
\hline 000051 & 100.30753 & 9.92890 & w & 223984075 & $\mathrm{Be}$ & 1.58 & 3.673 & $<1 \mathrm{E}-4$ & & 3.793 \\
\hline 000051 & 100.30753 & 9.92890 & $\mathrm{w}$ & 223984075 & $\mathrm{Be}$ & 1.58 & 3.221 & $2.5 \mathrm{E}-3$ & & "'”' \\
\hline 000055 & 100.39549 & 10.02980 & $\mathrm{w}$ & 616803382 & & 0.36 & & & & - \\
\hline 000056 & 100.47150 & 9.84649 & $\mathrm{c}$ & 223994760 & QPD & 1.16 & 5.833 & $<1 \mathrm{E}-4$ & 0.14 & 5.634 \\
\hline 000057 & 100.26642 & 9.96930 & w & 223981535 & $\mathrm{P}$ & 0.30 & 4.544 & $<1 \mathrm{E}-4$ & 0.01 & 4.557 \\
\hline 000058 & 100.53625 & 9.68922 & $\mathrm{c}$ & 616895632 & QPS & 1.29 & 2.142 & $<1 \mathrm{E}-4$ & 0.22 & - \\
\hline
\end{tabular}

Notes. ${ }^{(1)}$ Coordinates from the 2MASS survey. ${ }^{(2)}$ "c" = CTTS; "w" = WTTS. ${ }^{(3)}$ CoRoT light curve morphology class (cf. Appendix A; Cody et al. 2014): "B" = burster; "U" = unclassifiable variable type;"S" = stochastic; "N" = non-variable; "D" = dipper; "QPS" = quasi-periodic symmetric; "QPD" = quasi-periodic dipper; "P" = periodic; "MP" = multi-periodic; "EB" = eclipsing binary; "L" = long-timescale variable; "Be" = beats. (4) False Alarm Probability = fraction of times a periodogram power higher than that corresponding to the extracted period occurs, at the same frequency, among 10000 "noise-like" light curves built by dividing the original light curve in $12 \mathrm{~h}$-long segments and reassembling them in random order. ${ }^{(5)}$ Ratio of the amounts of effective light curve rms (i.e., $r m s^{2}-\sigma^{2}$ ) measured after and before subtracting the periodic trend from the light curve (see definition in Cody et al. 2014). A "-" indicates that the computed value of $Q$, associated with the period listed, is not reported because affected by systematics or by an erroneous estimate of the photometric uncertainty on the light curve. $Q$ is not reported for multi-periodic (MP) objects. ${ }^{(6)}$ From Affer et al. (2013). Blank space = aperiodic; “-" = object not present in Affer et al.'s sample. N.B.: multiple periods are not investigated in the study of Affer et al. (2013). (7) A period of $3.91 \mathrm{~d}$ is reported for this object in the analysis of McGinnis et al. (2015) and adopted in Sousa et al. (2016). The LSP obtained for this object presents a first peak at $P=3.91 \mathrm{~d}$ and a second, slightly lower peak at $P=7.83 \mathrm{~d}$. Conversely, the ACF analysis presents a slightly higher feature close to $P=8 \mathrm{~d}$, and a strong indication of periodicity at $P \sim 7.8 \mathrm{~d}$ is conveyed by the SL method, whereas no significant indication of periodicity at $P \sim 4 \mathrm{~d}$ appears in the latter. The light curve of this object appears as a sequence of dips which vary considerably in shape and especially depth along the monitored interval of time; however, the phased light curve at $P=7.83 \mathrm{~d}$ suggests that dips might come in pairs, alternating deeper and shallower minima, which may correspond, e.g., to a primary and a secondary opposite warps in the inner disk. Based notably on the SL result, we report here the longer periodicity of 7.83 d, although no decisive evidence in either direction can be achieved from our data. ${ }^{(8)}$ A periodic signal is present, but the waveform is strongly variable. ${ }^{(9)}$ The periodicity is only detected in part of the light curve. ${ }^{(10)}$ The results of the statistical tests do not strongly support the period detection here, but a visual inspection of the light curve indeed suggests the presence of a periodic pattern. ${ }^{(1)}$ The light curve seems to suggest a long periodicity of about $24.5 \mathrm{~d}$, but this is beyond what can be accurately probed here with our time coverage. ${ }^{(12)}$ Case similar, and more evident, to that of CSIMon-000296. The light curve exhibits a clear alternation of deeper and shallower minima, with the former being more jagged and the latter being sharper in shape; each pair of dips may represent two opposite warps in the inner disk. The periodogram provides a period indication at $P=4.48 \mathrm{~d}$, which is the value reported in McGinnis et al. (2015) for this object; conversely, a clear indication of periodicity at $P=9.102 \mathrm{~d}$ is provided by both the ACF and the SL analysis. We therefore consider this latter period estimate to provide a better match to the observed light curve for CSIMon-014132. ${ }^{(*)}$ More uncertain period estimate. ${ }^{(\#)}$ Spectroscopic binary. ${ }^{0}$ Cases with possible (uncertain) additional periodicities: CSIMon-000018 (3.612 d); CSIMon-000090 (3.571 d, $4.783 \mathrm{~d}$ ); CSIMon-000217 (7.463 d); CSIMon-000220 (7.675 d); CSIMon-000525 (hint of an additional long periodicity at $24 \mathrm{~d}$, but this is beyond what can be accurately probed here with our time coverage); CSIMon-000705 (9.389 d, only seen in the first part of the light curve); CSIMon-001067 (3.581 d); CSIMon-001303 (7.771 d). ${ }^{(a)}$ Jumps in the light curves affect the periodogram analysis. ${ }^{(b)}$ The periodogram peaks at a value corresponding to the half-period, whereas the correct periodicity is identified using the ACF and the SL methods. ${ }^{(c)}$ The periodogram peaks at a value corresponding to half that reported as period here; more ambiguous case. ${ }^{(d)}$ The light curve shows a spurious long-term trend that severely affects the LSP diagnostics for the detection of the short periodicity. 
Table F.1. continued.

\begin{tabular}{|c|c|c|c|c|c|c|c|c|c|c|}
\hline CSIMon-\# & $\mathrm{RA}^{1}$ & $\mathrm{Dec}^{1}$ & Class $^{2}$ & CoRoT_2011 & $\mathrm{LC}^{3}$ & $M_{\star}\left(M_{\odot}\right)$ & $P_{2011}^{\mathrm{CoRoT}}(\mathrm{d})$ & FAP $^{4}$ & $\overline{Q^{5}}$ & $P_{2008}^{\mathrm{CoRoT}}(\mathrm{d})^{6}$ \\
\hline 000060 & 100.31388 & 9.91415 & $\mathrm{~W}$ & 616826525 & $\mathrm{~N}$ & 0.29 & & & & - \\
\hline 000062 & 100.59126 & 9.80918 & $\mathrm{c}$ & 224003566 & $\mathrm{~N}$ & 0.90 & & & & \\
\hline 000063 & 100.29972 & 9.99479 & $\mathrm{c}$ & 616826518 & $\mathrm{~N}$ & 0.32 & & & & - \\
\hline 000066 & 100.26490 & 10.00982 & $\mathrm{~W}$ & 603431452 & QPS & 0.45 & 11.24 & $<1 \mathrm{E}-4$ & 0.07 & 11.25 \\
\hline 000067 & 100.48470 & 9.83495 & & 603420197 & $\mathrm{~N}$ & & & & & \\
\hline 000069 & 100.53066 & 9.82972 & & 223999063 & $\mathrm{~N}$ & & & & & \\
\hline 000071 & 100.25105 & 9.98046 & w & 223980621 & QPS & 1.69 & 5.41 & $<1 \mathrm{E}-4$ & 0.32 & 3.049 \\
\hline 000075 & 100.29829 & 10.03990 & W & 223983509 & $\mathrm{P}$ & 1.48 & 2.385 & $<1 \mathrm{E}-4$ & 0.09 & 2.39 \\
\hline 000080 & 100.32480 & 10.06725 & $\mathrm{c}$ & 616803514 & $\mathrm{U}$ & 0.28 & & & & - \\
\hline 000087 & 100.27743 & 9.59585 & w & 602083898 & $\mathrm{~L}$ & 1.07 & & & & - \\
\hline 000088 & 100.39181 & 9.35371 & $\mathrm{~W}$ & 616969757 & $\mathrm{U}$ & 0.32 & & & & - \\
\hline 000090 & 100.28733 & 9.56278 & $\mathrm{c}$ & 616919796 & $\mathrm{Be}$ & 0.30 & 4.115 & $<1 \mathrm{E}-4$ & & 4.042 \\
\hline 000095 & 100.18384 & 9.39872 & W & 223976494 & $\mathrm{P}$ & 1.00 & 2.256 & $<1 \mathrm{E}-4$ & 0.10 & 2.267 \\
\hline 000096 & 100.24432 & 9.76515 & W & 616872594 & & 0.63 & & & & - \\
\hline 000098 & 100.22645 & 9.33462 & & 223979114 & & & 0.767 & $1.0 \mathrm{E}-2^{a}$ & -0.20 & - \\
\hline 000102 & 100.18016 & 9.52084 & w & 400007786 & QPS & 0.30 & 8.9 & $<1 \mathrm{E}-4$ & -0.07 & - \\
\hline 000103 & 100.24807 & 9.58637 & $\mathrm{c}$ & 223980447 & QPS & 0.93 & 3.348 & $<1 \mathrm{E}-4$ & 0.21 & 1.675 \\
\hline 000105 & 100.21000 & 9.81390 & W & 616849431 & $\mathrm{~N}$ & 1.28 & & & & - \\
\hline 000108 & 100.31183 & 9.54330 & w & 616919655 & QPS & 0.30 & 4.058 & $5.0 \mathrm{E}-1^{b}$ & -0.17 & - \\
\hline 000109 & 100.40601 & 9.62440 & $\mathrm{c}$ & 223990338 & $\mathrm{~N}$ & & & & & - \\
\hline 000111 & 100.24379 & 9.55883 & & 223980201 & $\mathrm{U}$ & & & & & - \\
\hline 000117 & 100.22555 & 9.81206 & $\mathrm{c}$ & 602095753 & $\mathrm{~B}$ & 0.32 & & & & - \\
\hline 000119 & 100.33749 & 9.56006 & $\mathrm{c}$ & 223985987 & $\mathrm{QP}$ & 0.91 & 3.31 & $<1 \mathrm{E}-4$ & 0.63 & 3.308 \\
\hline 000122 & 100.44630 & 9.63463 & W & 223993002 & QPS & 0.95 & 5.319 & $<1 \mathrm{E}-4$ & 0.05 & - \\
\hline 000123 & 100.28419 & 9.56926 & w & 616919795 & $\mathrm{~N}$ & 0.31 & & & & - \\
\hline 000126 & 100.24099 & 9.68894 & $\mathrm{c}$ & 616895876 & D & 0.62 & & & & - \\
\hline 000131 & 100.20535 & 9.39732 & $\mathrm{c}$ & 616969822 & $\mathrm{U}$ & 0.78 & 12.867 & $<1 \mathrm{E}-4$ & 0.44 & - \\
\hline 000134 & 100.31009 & 9.44953 & $\mathrm{c}$ & 603808964 & $\mathrm{QP}$ & 0.28 & 3.017 & $3.0 \mathrm{E}-2$ & - & \\
\hline 000139 & 100.22364 & 9.96668 & w & 616826651 & QPS & 0.45 & 8.042 & $<1 \mathrm{E}-4$ & 0.26 & - \\
\hline 000141 & 100.26129 & 9.38862 & & 603808908 & QPS & & 4.652 & $<1 \mathrm{E}-4$ & 0.24 & - \\
\hline 000145 & 100.34776 & 9.76631 & w & 616872632 & U & 0.71 & & & & - \\
\hline 000146 & 100.19060 & 9.97463 & w & 223976871 & $\mathrm{~N}$ & 0.85 & & & & - \\
\hline 000153 & 100.24963 & 9.78457 & $\mathrm{c}$ & 400007889 & QPS & 0.29 & 1.896 & $<1 \mathrm{E}-4$ & 0.35 & - \\
\hline 000158 & 100.18684 & 9.77732 & w & 605538580 & & 0.95 & 10.108 & $<1 \mathrm{E}-4$ & -0.45 & - \\
\hline 000159 & 100.21445 & 9.62068 & W & 602087947 & QPS & 0.45 & 8.798 & $<1 \mathrm{E}-4$ & 0.14 & - \\
\hline 000160 & 100.24929 & 9.86359 & w & 605538519 & QPS & 0.36 & 1.792 & $<1 \mathrm{E}-4$ & 0.18 & 1.805 \\
\hline 000164 & 100.26880 & 9.50376 & W & 616919778 & MP & 0.29 & 0.669 & $<1 \mathrm{E}-4$ & & - \\
\hline 000164 & 100.26880 & 9.50376 & w & 616919778 & MP & 0.29 & 0.904 & $7.0 \mathrm{E}-4$ & & - \\
\hline 000168 & 100.42866 & 9.41899 & $\mathrm{c}$ & 223991832 & $\mathrm{QP}$ & 0.90 & 10.019 & $<1 \mathrm{E}-4$ & 0.6 & 8.608 \\
\hline 000169 & 100.45027 & 9.71203 & W & 223993277 & QPS & 1.55 & 1.173 & $<1 \mathrm{E}-4$ & 0.21 & 1.184 \\
\hline 000172 & 100.29297 & 9.36376 & w & 616969725 & $\mathrm{U}$ & 0.33 & & & & 8.08 \\
\hline 000176 & 100.21752 & 9.87531 & w & 602266743 & QPS & 0.36 & 7.694 & $<1 \mathrm{E}-4$ & 0.03 & - \\
\hline 000177 & 100.27584 & 9.60638 & $\mathrm{c}$ & 223982136 & QPS & 1.48 & 3.029 & $<1 \mathrm{E}-4$ & 0.22 & 3.018 \\
\hline 000183 & 100.31879 & 9.43564 & & 602079851 & U & & 6.273 & $<1 \mathrm{E}-4$ & 0.52 & - \\
\hline 000184 & 100.33018 & 9.51354 & & 616919664 & $\mathrm{~N}$ & 0.16 & & & & - \\
\hline 000185 & 100.41154 & 9.53663 & $\mathrm{c}$ & 616919566 & $\mathrm{~S}$ & 1.22 & & & & \\
\hline 000188 & 100.25719 & 9.93097 & W & 602099710 & QPS & 0.28 & 1.74 & $1.0 \mathrm{E}-2$ & - & - \\
\hline 000192 & 100.20837 & 9.74840 & $\mathrm{c}$ & 616872583 & $\mathrm{~N}$ & 0.30 & & & & - \\
\hline 000198 & 100.33183 & 9.52900 & w & 223985611 & QPS & 1.19 & 4.996 & $2.0 \mathrm{E}-1^{b}$ & 0.80 & 4.94 \\
\hline 000200 & 100.28339 & 9.51120 & w & 616919794 & QPS & 1.12 & 1.929 & $<1 \mathrm{E}-4$ & & - \\
\hline 000204 & 100.19670 & 9.88588 & & 602095749 & $\mathrm{~N}$ & 0.37 & & & & - \\
\hline 000206 & 100.24747 & 9.95985 & w & 616826502 & $\mathrm{~N}$ & 0.70 & & & & - \\
\hline 000207 & 100.24598 & 9.81841 & $\mathrm{~W}$ & 602095756 & QPS & 0.34 & 1.996 & $<1 \mathrm{E}-4$ & 0.28 & - \\
\hline 000216 & 100.26538 & 9.47233 & $\mathrm{~W}$ & 616944007 & & 0.45 & & & & - \\
\hline 000217 & 100.27903 & 9.68180 & $\mathrm{~W}$ & 400007956 & MP? & 0.27 & 1.262 & $9.0 \mathrm{E}-2$ & & 1.26 \\
\hline 000219 & 100.32868 & 9.59839 & $\mathrm{c}$ & 616919663 & $\mathrm{~N}$ & 0.23 & & & & - \\
\hline 000220 & 100.35228 & 9.62653 & $\mathrm{c}$ & 616895930 & MP? & 0.30 & 0.75 & $<1 \mathrm{E}-4$ & 0.52 & \\
\hline 000223 & 100.23094 & 9.62326 & W & 602087949 & $\mathrm{P}$ & 0.91 & 1.9 & $<1 \mathrm{E}-4$ & 0.10 & - \\
\hline 000226 & 100.27236 & 9.55374 & $\mathrm{~W}$ & 603402475 & $\mathrm{P}$ & 1.20 & 1.206 & $<1 \mathrm{E}-4$ & 0.00 & - \\
\hline 000227 & 100.22477 & 9.84948 & w & 605538529 & $\mathrm{~N}$ & 0.26 & & & & \\
\hline
\end{tabular}


Table F.1. continued.

\begin{tabular}{|c|c|c|c|c|c|c|c|c|c|c|}
\hline CSIMon-\# & $\mathrm{RA}^{1}$ & $\operatorname{Dec}^{1}$ & Class $^{2}$ & CoRoT_2011 & $\mathrm{LC}^{3}$ & $M_{\star}\left(M_{\odot}\right)$ & $P_{2011}^{\mathrm{CoRoT}}(\mathrm{d})$ & $\mathrm{FAP}^{4}$ & $\mathrm{Q}^{5}$ & $P_{2008}^{\mathrm{CoRoT}}(\mathrm{d})^{6}$ \\
\hline 000235 & 100.24226 & 9.87655 & & 605538496 & $\mathrm{U}$ & & & & & - \\
\hline 000236 & 100.26056 & 9.58217 & $\mathrm{w}$ & 223981174 & $\mathrm{P}$ & 1.37 & 1.979 & $<1 \mathrm{E}-4$ & 0.11 & 1.974 \\
\hline 000237 & 100.29033 & 9.41520 & w & 616944029 & $\mathrm{P}$ & 0.45 & 3.381 & $<1 \mathrm{E}-4$ & 0.13 & - \\
\hline 000241 & 100.34598 & 9.45741 & $\mathrm{w}$ & 223986498 & QPS & 1.85 & 3.25 & $<1 \mathrm{E}-4$ & 0.16 & 3.206 \\
\hline 000242 & 100.29940 & 9.44206 & $\mathrm{c}$ & 602079796 & $\mathrm{D}$ & 0.45 & & & & \\
\hline 000247 & 100.28035 & 9.83240 & $\mathrm{w}$ & 616849465 & & 0.23 & & & & - \\
\hline 000250 & 100.25206 & 9.75086 & $\mathrm{c}$ & 223980688 & QPD & 1.35 & 8.929 & $<1 \mathrm{E}-4$ & 0.37 & \\
\hline 000253 & 100.30370 & 9.76689 & w & 616872613 & $\mathrm{~N}$ & 0.25 & & & & - \\
\hline 000255 & 100.42801 & 9.71574 & $\mathrm{w}$ & 223991789 & $\mathrm{P}$ & 0.62 & 3.927 & $<1 \mathrm{E}-4$ & 0.14 & 3.956 \\
\hline 000256 & 100.43427 & 9.41733 & $\mathrm{~W}$ & 223992193 & EB & 0.36 & 3.874 & $6.0 \mathrm{E}-3^{b}$ & 0.45 & - \\
\hline 000263 & 100.26081 & 9.58698 & w & 602083896 & QPS & 1.20 & 4.287 & $<1 \mathrm{E}-4$ & -0.07 & - \\
\hline 000273 & 100.32653 & 9.66143 & $\mathrm{c}$ & 616895921 & $\mathrm{~N}$ & 0.45 & & & & - \\
\hline 000274 & 100.27864 & 9.38924 & $\mathrm{w}$ & 602075361 & QPS & 2.20 & 12.123 & $<1 \mathrm{E}-4$ & - & 11.92 \\
\hline 000279 & 100.33823 & 9.53743 & $\mathrm{c}$ & 603402480 & QPS & 0.32 & 7.935 & $<1 \mathrm{E}-4$ & 0.45 & - \\
\hline 000280 & 100.17088 & 9.46509 & $\mathrm{c}$ & 616944098 & $\mathrm{~N}$ & 0.99 & & & & \\
\hline 000289 & 100.19650 & 9.48049 & & 616943962 & $\mathrm{U}$ & & & & & \\
\hline 000290 & 100.24440 & 9.60366 & $\mathrm{c}$ & 223980233 & QPS & 0.25 & 5.940 & $<1 \mathrm{E}-4$ & & \\
\hline 000292 & 100.44757 & 9.70010 & $\mathrm{w}$ & 223993084 & QPS & 0.45 & 6.573 & $<1 \mathrm{E}-4$ & 0.11 & 6.456 \\
\hline 000294 & 100.26819 & 9.45852 & $\mathrm{w}$ & 616944010 & & 0.28 & & & & - \\
\hline $000296^{7}$ & 100.21079 & 9.91592 & $\mathrm{c}$ & 602099706 & QPD & 1.42 & 7.83 & $<1 \mathrm{E}-4$ & 0.57 & \\
\hline 000297 & 100.18817 & 9.47901 & $\mathrm{c}$ & 223976747 & $\mathrm{D}$ & 1.42 & & & & 3.173 \\
\hline 000298 & 100.27368 & 9.90520 & $\mathrm{w}$ & 605538656 & $\mathrm{Be}$ & 0.45 & 1.308 & $<1 \mathrm{E}-4$ & 0.33 & 1.289 \\
\hline 000298 & 100.27368 & 9.90520 & $\mathrm{w}$ & 605538656 & $\mathrm{Be}$ & 0.45 & 1.246 & $<1 \mathrm{E}-4$ & & "" \\
\hline 000305 & 100.23951 & 9.91596 & & 605538647 & $\mathrm{~N}$ & & & & & - \\
\hline 000306 & 100.30207 & 9.77236 & $\mathrm{w}$ & 616872612 & $\mathrm{Be}$ & 0.23 & 0.452 & $<1 \mathrm{E}-4$ & 0.14 & - \\
\hline 000306 & 100.30207 & 9.77236 & $\mathrm{w}$ & 616872612 & $\mathrm{Be}$ & 0.23 & 0.425 & $1.0 \mathrm{E}-2$ & & - \\
\hline 000311 & 100.48245 & 9.66614 & $\mathrm{~W}$ & 616895733 & QPS & 0.32 & 6.497 & $<1 \mathrm{E}-4$ & -0.15 & - \\
\hline 000314 & 100.18579 & 9.54061 & $\mathrm{c}$ & 616919732 & QPD & 0.29 & 3.279 & $2.0 \mathrm{E}-4$ & 0.80 & 6.3 \\
\hline 000319 & 100.21445 & 9.52969 & $\mathrm{~W}$ & 616919745 & $\mathrm{~N}$ & 0.33 & & & & - \\
\hline 000325 & 100.24726 & 9.92227 & $\mathrm{c}$ & 605538641 & $\mathrm{D}$ & 1.99 & & & & - \\
\hline 000326 & 100.24511 & 9.65520 & $\mathrm{c}$ & 223980258 & QP & 0.66 & 6.642 & $<1 \mathrm{E}-4$ & 0.6 & 6.99 \\
\hline 000328 & 100.36252 & 9.50364 & $\mathrm{c}$ & 400007735 & $\mathrm{~N}$ & 0.45 & & & & 9.996 \\
\hline 000330 & 100.38172 & 9.80911 & w & 223988742 & QPS & 1.55 & 5.054 & $<1 \mathrm{E}-4$ & 0.02 & 5.025 \\
\hline 000335 & 100.40540 & 9.75182 & $\mathrm{c}$ & 223990299 & $\mathrm{P}$ & 1.40 & 4.577 & $<1 \mathrm{E}-4$ & 0.00 & 4.469 \\
\hline 000338 & 100.47703 & 9.48775 & & 223995167 & $\mathrm{~N}$ & & & & & \\
\hline 000339 & 100.39308 & 9.43150 & $\mathrm{~W}$ & 603396408 & $\mathrm{U}$ & 0.30 & & & & - \\
\hline 000340 & 100.48303 & 9.67968 & $\mathrm{c}$ & 616895736 & QP & 0.33 & 1.225 & $3.0 \mathrm{E}-3$ & 0.53 & - \\
\hline 000341 & 100.22608 & 9.82232 & $\mathrm{c}$ & 616849439 & $\mathrm{~B}$ & 0.53 & & & & \\
\hline 000342 & 100.23227 & 9.77934 & $\mathrm{c}$ & 616872592 & $\mathrm{D}$ & 0.25 & & & & - \\
\hline 000344 & 100.28963 & 9.59041 & $\mathrm{w}$ & 602083902 & $\mathrm{P}$ & 0.36 & 0.856 & $<1 \mathrm{E}-4$ & 0.10 & - \\
\hline 000345 & 100.18776 & 9.85769 & w & 616849563 & & 0.35 & & & & - \\
\hline 000346 & 100.28789 & 9.50255 & $\mathrm{c}$ & 603402478 & $\mathrm{~S}$ & 0.70 & & & & - \\
\hline 000348 & 100.20871 & 9.87432 & w & 616849429 & QPS & 0.25 & 5.189 & $1.0 \mathrm{E}-4$ & 0.62 & - \\
\hline 000351 & 100.34502 & 9.49416 & $\mathrm{w}$ & 616943891 & MP & 0.93 & 10.488 & $<1 \mathrm{E}-4$ & & - \\
\hline 000351 & 100.34502 & 9.49416 & $\mathrm{w}$ & 616943891 & MP & 0.93 & 1.064 & $4.0 \mathrm{E}-2$ & 0.93 & - \\
\hline 000354 & 100.28680 & 9.39528 & $\mathrm{w}$ & 223982779 & QPS & 0.55 & 1.727 & $<1 \mathrm{E}-4$ & 0.46 & 1.882 \\
\hline 000356 & 100.27111 & 9.82300 & $\mathrm{c}$ & 616849458 & $\mathrm{~N}$ & 0.20 & & & & 4.364 \\
\hline 000357 & 100.27396 & 9.51698 & $\mathrm{c}$ & 616919781 & $\mathrm{~N}$ & 1.20 & & & & - \\
\hline 000358 & 100.27803 & 9.79099 & $\mathrm{c}$ & 400007959 & QPD & 0.29 & 5.821 & $<1 \mathrm{E}-4$ & 0.15 & 5.738 \\
\hline 000368 & 100.24334 & 9.45697 & w & 616943994 & $\mathrm{U}$ & 0.24 & 1.031 & $2.0 \mathrm{E}-1$ & & 1.029 \\
\hline 000370 & 100.23663 & 9.63025 & $\mathrm{c}$ & 223979728 & QPS & 1.20 & 11.838 & $<1 \mathrm{E}-4$ & 0.32 & \\
\hline 000372 & 100.33561 & 9.75990 & w & 223985845 & $\mathrm{P}$ & 1.26 & 2.567 & $<1 \mathrm{E}-4$ & 0.12 & 2.604 \\
\hline 000375 & 100.42884 & 9.39107 & w & 616969611 & QPS & 0.31 & 8.725 & $<1 \mathrm{E}-4$ & 0.43 & - \\
\hline 000377 & 100.45361 & 9.72037 & $\mathrm{w}$ & 223993499 & $\mathrm{U}$ & 1.98 & & & & \\
\hline 000378 & 100.22048 & 9.74841 & $\mathrm{c}$ & 616872605 & QPS & 1.06 & 11.029 & $<1 \mathrm{E}-4$ & 0.21 & - \\
\hline $000379^{8}$ & 100.27069 & 9.84613 & $\mathrm{c}$ & 223981811 & $\mathrm{D}$ & 1.60 & 3.66 & $<1 \mathrm{E}-4$ & 0.78 & \\
\hline 000383 & 100.23242 & 9.67172 & $\mathrm{w}$ & 400007851 & QP & 0.32 & 1.027 & $2.0 \mathrm{E}-1$ & - & - \\
\hline 000389 & 100.20319 & 9.54513 & $\mathrm{~W}$ & 616919741 & $\mathrm{U}$ & 1.25 & & & & - \\
\hline 000394 & 100.28234 & 9.68749 & $\mathrm{w}$ & 616895903 & QPS & 0.62 & 1.963 & $<1 \mathrm{E}-4$ & 0.36 & - \\
\hline
\end{tabular}


Table F.1. continued.

\begin{tabular}{|c|c|c|c|c|c|c|c|c|c|c|}
\hline CSIMon-\# & $\mathrm{RA}^{1}$ & $\operatorname{Dec}^{1}$ & Class $^{2}$ & CoRoT_2011 & $\mathrm{LC}^{3}$ & $M_{\star}\left(M_{\odot}\right)$ & $P_{2011}^{\mathrm{CoRoT}}(\mathrm{d})$ & $\mathrm{FAP}^{4}$ & $\mathrm{Q}^{5}$ & $P_{2008}^{\text {CoRoT }}(\mathrm{d})^{6}$ \\
\hline 000395 & 100.29228 & 9.52463 & $\mathrm{~W}$ & 616919644 & QPS & 0.28 & 1.654 & $2.0 \mathrm{E}-2$ & - & - \\
\hline 000397 & 100.29497 & 9.77812 & W & 400007809 & $\mathrm{U}$ & 0.27 & & & & \\
\hline 000406 & 100.24864 & 9.47881 & $\mathrm{c}$ & 616943998 & B & 1.13 & 6.631 & $<1 \mathrm{E}-4$ & 0.50 & - \\
\hline 000407 & 100.34179 & 9.85350 & $\mathrm{w}$ & 616849492 & QPS & 0.72 & 4.504 & $1.0 \mathrm{E}-4$ & 0.41 & - \\
\hline 000410 & 100.27674 & 9.90298 & & 605538659 & $\mathrm{P}$ & 0.32 & 0.833 & $<1 \mathrm{E}-4$ & -1.29 & - \\
\hline 000412 & 100.19630 & 9.54449 & $\mathrm{c}$ & 616919737 & B & 0.45 & 6.679 & $<1 \mathrm{E}-4$ & 0.51 & - \\
\hline 000413 & 100.44350 & 9.71856 & W & 603414387 & $\mathrm{P}$ & 0.95 & 4.281 & $<1 \mathrm{E}-4$ & -0.01 & - \\
\hline 000415 & 100.18274 & 9.80848 & w & 616849557 & $\mathrm{P}$ & 0.45 & 0.961 & $<1 \mathrm{E}-4$ & 0.42 & - \\
\hline 000420 & 100.22579 & 9.93111 & W & 602099707 & QPS & 0.66 & 8.1 & $<1 \mathrm{E}-4$ & -0.42 & - \\
\hline 000423 & 100.31191 & 9.43199 & $\mathrm{c}$ & 602079850 & $\mathrm{D}$ & 0.26 & & & & - \\
\hline 000424 & 100.31334 & 9.63267 & $\mathrm{c}$ & 616895917 & $\mathrm{~N}$ & 0.45 & & & & - \\
\hline 000425 & 100.31951 & 9.49786 & $\mathrm{c}$ & 616943882 & $\mathrm{~S}$ & 1.22 & 7.51 & $8.0 \mathrm{E}-3$ & 0.5 & - \\
\hline 000426 & 100.33108 & 9.50799 & $\mathrm{c}$ & 616919665 & $\mathrm{U}$ & 1.20 & & & & - \\
\hline 000427 & 100.17683 & 9.53906 & w & 223976099 & QPS & 1.12 & 14.813 & $<1 \mathrm{E}-4$ & 0.22 & 14.17 \\
\hline 000430 & 100.23655 & 9.50419 & $\mathrm{w}$ & 223979719 & $\mathrm{Be}$ & 0.52 & 0.533 & $<1 \mathrm{E}-4$ & 0.27 & - \\
\hline 000430 & 100.23655 & 9.50419 & W & 223979719 & $\mathrm{Be}$ & 0.52 & 0.552 & $<1 \mathrm{E}-4$ & & - \\
\hline 000433 & 100.25462 & 9.58117 & $\mathrm{c}$ & 616919770 & QPD & 0.44 & 9.798 & $<1 \mathrm{E}-4$ & 0.50 & - \\
\hline 000434 & 100.26691 & 9.58924 & $\mathrm{c}$ & 616919776 & MP & 0.33 & 7.485 & $<1 \mathrm{E}-4$ & & - \\
\hline 000434 & 100.26691 & 9.58924 & $\mathrm{c}$ & 616919776 & MP & 0.33 & 0.725 & $1.0 \mathrm{E}-4$ & 0.83 & - \\
\hline 000438 & 100.26427 & 9.50139 & $\mathrm{~W}$ & 616919773 & $\mathrm{P}$ & 0.34 & 1.308 & $<1 \mathrm{E}-4$ & -0.33 & - \\
\hline 000440 & 100.41271 & 9.49394 & w & 223990764 & $\mathrm{U}$ & 0.74 & & & & \\
\hline 000441 & 100.24206 & 9.61485 & $\mathrm{c}$ & 223980048 & QPD & 0.36 & & & & 12.5 \\
\hline 000443 & 100.36481 & 9.53213 & w & 223987667 & $\mathrm{U}$ & 1.30 & & & & - \\
\hline 000444 & 100.43574 & 9.70346 & w & 223992277 & QPS & 0.36 & 10.246 & $<1 \mathrm{E}-4$ & 0.00 & - \\
\hline 000445 & 100.51066 & 9.61458 & W & 223997570 & MP? & 0.95 & 3.651 & $<1 \mathrm{E}-4$ & & 3.66 \\
\hline $000448^{9}$ & 100.26502 & 9.50808 & $\mathrm{c}$ & 602083897 & $\mathrm{U}$ & 0.25 & 4.731 & $5.0 \mathrm{E}-4$ & 0.78 & 9.73 \\
\hline 000450 & 100.49184 & 9.71841 & $\mathrm{w}$ & 602091914 & $\mathrm{P}$ & 0.94 & 2.102 & $<1 \mathrm{E}-4$ & 0.07 & - \\
\hline 000451 & 100.46447 & 9.73602 & w & 616872431 & QPS & 0.45 & 4.515 & $<1 \mathrm{E}-4$ & 0.21 & - \\
\hline 000454 & 100.26513 & 9.60130 & & 616895890 & $\mathrm{~N}$ & & & & & - \\
\hline 000456 & 100.21475 & 9.72339 & $\mathrm{c}$ & 616872585 & QPD & 1.41 & 5.054 & $<1 \mathrm{E}-4$ & 0.62 & - \\
\hline 000457 & 100.27805 & 9.57935 & $\mathrm{c}$ & 616919789 & S & 1.82 & & & & \\
\hline 000461 & 100.47901 & 9.50181 & w & 616919638 & QPS & 0.35 & 3.429 & $<1 \mathrm{E}-4$ & 0.26 & - \\
\hline $000462^{10}$ & 100.17576 & 9.56040 & $\mathrm{c}$ & 223976028 & $\mathrm{D}$ & 1.60 & 1.913 & $5.0 \mathrm{E}-2$ & & \\
\hline $000468^{\#}$ & 100.28694 & 9.76696 & W & 223982794 & $\mathrm{Be}$ & 1.30 & 4.146 & $<1 \mathrm{E}-4$ & & - \\
\hline $000468^{\#}$ & 100.28694 & 9.76696 & $\mathrm{~W}$ & 223982794 & $\mathrm{Be}$ & 1.30 & 4.933 & $<1 \mathrm{E}-4$ & & - \\
\hline 000469 & 100.17142 & 9.56607 & $\mathrm{c}$ & 602083890 & $\mathrm{~S}$ & 0.71 & & & & - \\
\hline 000470 & 100.18431 & 9.89426 & & 602095747 & $\mathrm{~N}$ & & & & & - \\
\hline 000474 & 100.27837 & 9.45892 & $\mathrm{c}$ & 603396438 & B & & & & & - \\
\hline 000477 & 100.31560 & 9.43806 & w & 223984608 & $\mathrm{P}$ & 1.22 & 6.227 & $<1 \mathrm{E}-4$ & 0.02 & 6.098 \\
\hline 000484 & 100.27258 & 9.56930 & $\mathrm{c}$ & 616919779 & & 0.13 & 19.5 & $<1 \mathrm{E}-4$ & & - \\
\hline 000486 & 100.26684 & 9.81911 & w & 602095758 & QPS & 0.69 & 12.385 & $<1 \mathrm{E}-4$ & 0.02 & - \\
\hline 000488 & 100.24094 & 9.94167 & w & 223979980 & & 1.60 & 0.583 & $<1 \mathrm{E}-4$ & - & 0.577 \\
\hline 000491 & 100.23401 & 9.60857 & $\mathrm{c}$ & 616895873 & $\mathrm{~L}$ & 1.70 & & & & - \\
\hline 000493 & 100.35452 & 9.60004 & w & 400008004 & & 0.27 & & & & 3.19 \\
\hline 000497 & 100.25919 & 9.86446 & $\mathrm{w}$ & 616849449 & $\mathrm{P}$ & 2.09 & 9.988 & $<1 \mathrm{E}-4$ & 0.06 & 10.0 \\
\hline 000498 & 100.19793 & 9.82470 & $\mathrm{c}$ & 616849574 & QPD & 1.90 & 4.3 & $<1 \mathrm{E}-4$ & 0.15 & 8.53 \\
\hline 000499 & 100.26896 & 9.42175 & $\mathrm{w}$ & 616944012 & $\mathrm{~N}$ & 0.25 & & & & - \\
\hline 000500 & 100.23279 & 9.85847 & & 616849441 & $\mathrm{U}$ & 0.38 & & & & - \\
\hline 000510 & 100.26787 & 9.41449 & $\mathrm{c}$ & 602079845 & B & 0.62 & & & & 14.99 \\
\hline 000515 & 100.40097 & 9.65568 & w & 223989989 & $\mathrm{P}$ & 0.72 & 6.542 & $<1 \mathrm{E}-4$ & -0.08 & 6.547 \\
\hline 000517 & 100.26964 & 9.60742 & w & 223981753 & MP & 2.40 & 2.772 & $4.0 \mathrm{E}-4$ & 0.36 & 2.971 \\
\hline 000517 & 100.26964 & 9.60742 & w & 223981753 & MP & 2.40 & 3.004 & $1.0 \mathrm{E}-3$ & 0.36 & "" \\
\hline 000518 & 100.25705 & 9.80614 & $\mathrm{w}$ & 223980989 & QPS & 1.21 & 6.546 & $<1 \mathrm{E}-4$ & 0.51 & - \\
\hline 000519 & 100.29133 & 9.50560 & W & 616919643 & QPS & 0.34 & 6.008 & $6.0 \mathrm{E}-4$ & - & - \\
\hline 000524 & 100.26806 & 9.80614 & w & 616849453 & $\mathrm{P}$ & 1.31 & 5.152 & $<1 \mathrm{E}-4$ & 0.06 & - \\
\hline 000525 & 100.21323 & 9.74612 & $\mathrm{c}$ & 223978308 & QPD,MP? & 1.80 & 1.992 & $<1 \mathrm{E}-4$ & & 5.374 \\
\hline 000529 & 100.21334 & 9.46610 & $\mathrm{w}$ & 616943973 & $\mathrm{QP}$ & 0.31 & 7.163 & $<1 \mathrm{E}-4$ & - & - \\
\hline 000530 & 100.33079 & 9.36309 & $\mathrm{c}$ & 602075358 & $\mathrm{~N}$ & 0.82 & & & & - \\
\hline
\end{tabular}


Table F.1. continued.

\begin{tabular}{|c|c|c|c|c|c|c|c|c|c|c|}
\hline CSIMon-\# & $\mathrm{RA}^{1}$ & $\operatorname{Dec}^{1}$ & Class $^{2}$ & CoRoT_2011 & $\mathrm{LC}^{3}$ & $M_{\star}\left(M_{\odot}\right)$ & $P_{2011}^{\text {CoRoT }}(\mathrm{d})$ & $\mathrm{FAP}^{4}$ & $\mathrm{Q}^{5}$ & $P_{2008}^{\mathrm{CoRoT}}(\mathrm{d})^{6}$ \\
\hline 000536 & 100.21666 & 9.75132 & W & 400007394 & QPS & 0.45 & 3.402 & $<1 \mathrm{E}-4$ & - & 3.443 \\
\hline 000548 & 100.28568 & 9.71432 & $\mathrm{~W}$ & 603414376 & QPS & 0.45 & 10.7 & $<1 \mathrm{E}-4$ & -1.46 & - \\
\hline 000555 & 100.35191 & 9.54589 & $\mathrm{~W}$ & 616919676 & QP & 0.19 & 1.048 & $8.0 \mathrm{E}-4$ & 0.62 & - \\
\hline 000558 & 100.41561 & 9.67442 & $\mathrm{c}$ & 223990964 & QPS & 1.60 & 11.708 & $<1 \mathrm{E}-4$ & 0.43 & 10.17 \\
\hline 000559 & 100.35105 & 9.53172 & $\mathrm{~W}$ & 223986811 & & 0.95 & 7.956 & $<1 \mathrm{E}-4$ & 0.47 & 7.92 \\
\hline 000560 & 100.31547 & 9.63841 & $\mathrm{w}$ & 223984600 & QPS & 1.34 & 5.313 & $<1 \mathrm{E}-4$ & -0.13 & 5.343 \\
\hline 000563 & 100.42436 & 9.55060 & $\mathrm{~W}$ & 616919579 & $\mathrm{~N}$ & 0.23 & & & & - \\
\hline 000565 & 100.25325 & 9.85621 & $\mathrm{w}$ & 616849446 & $\mathrm{P}$ & 1.35 & 4.375 & $<1 \mathrm{E}-4$ & 0.02 & 4.344 \\
\hline 000566 & 100.21982 & 9.71678 & $\mathrm{c}$ & 400007955 & $\mathrm{U}$ & 0.26 & & & & \\
\hline 000567 & 100.23500 & 9.59813 & $\mathrm{c}$ & 616919752 & $\mathrm{~B}$ & 1.45 & & & & - \\
\hline 000568 & 100.22405 & 9.51084 & $\mathrm{w}$ & 223978947 & $\mathrm{U}$ & 1.80 & & & & 8.5 \\
\hline 000574 & 100.21192 & 9.93140 & $\mathrm{w}$ & 223978227 & $\mathrm{P}$ & 0.70 & 3.794 & $<1 \mathrm{E}-4$ & 0.15 & 3.779 \\
\hline 000577 & 100.43258 & 9.68055 & $\mathrm{c}$ & 616895846 & S & 1.62 & & & & - \\
\hline 000578 & 100.45846 & 9.49228 & $\mathrm{c}$ & 223993840 & $\mathrm{P}$ & 0.94 & 3.269 & $<1 \mathrm{E}-4$ & 0.13 & 3.25 \\
\hline 000581 & 100.20040 & 9.89427 & w & 602095750 & QPS & 1.00 & 5.521 & $5.0 \mathrm{E}-4$ & 0.36 & - \\
\hline 000583 & 100.22171 & 9.49839 & $\mathrm{~W}$ & 400008031 & & 0.28 & & & & - \\
\hline 000586 & 100.24166 & 9.69209 & & 605538448 & $\mathrm{U}$ & & & & & - \\
\hline 000590 & 100.24549 & 9.48131 & $\mathrm{c}$ & 603396403 & $\mathrm{~S}$ & 0.45 & & & & - \\
\hline 000596 & 100.44768 & 9.63129 & $\mathrm{w}$ & 602087963 & $\mathrm{P}$ & 0.45 & 9.142 & $<1 \mathrm{E}-4$ & 0.08 & - \\
\hline 000598 & 100.24721 & 9.77128 & $\mathrm{c}$ & 616872597 & B & 0.45 & & & & - \\
\hline 000604 & 100.27124 & 9.86236 & $\mathrm{~W}$ & 616849455 & $\mathrm{P}$ & 1.20 & 9.958 & $<1 \mathrm{E}-4$ & 0.03 & - \\
\hline 000606 & 100.31028 & 9.55595 & W & 223984253 & $\mathrm{P}$ & 1.20 & 10.798 & $<1 \mathrm{E}-4$ & 0.05 & 10.42 \\
\hline 000607 & 100.31516 & 9.44262 & $\mathrm{~W}$ & 223984572 & $\mathrm{Be}$ & 0.28 & 1.581 & $<1 \mathrm{E}-4$ & 0.31 & \\
\hline 000607 & 100.31516 & 9.44262 & $\mathrm{w}$ & 223984572 & $\mathrm{Be}$ & 0.28 & 1.277 & $2.0 \mathrm{E}-2$ & & \\
\hline 000609 & 100.41503 & 9.55553 & $\mathrm{~W}$ & 603402484 & $\mathrm{~N}$ & 0.29 & & & & - \\
\hline 000610 & 100.18907 & 9.63095 & $\mathrm{w}$ & 616896016 & $\mathrm{~N}$ & 0.67 & & & & - \\
\hline 000612 & 100.24250 & 9.92905 & $\mathrm{w}$ & 602266739 & $\mathrm{P}$ & 0.30 & 4.304 & $<1 \mathrm{E}-4$ & 0.05 & - \\
\hline 000613 & 100.27406 & 9.80486 & $\mathrm{c}$ & 616849463 & $\mathrm{~S}$ & 0.78 & & & & - \\
\hline 000614 & 100.17435 & 9.69406 & w & 616896008 & $\mathrm{~N}$ & 0.27 & & & & 1.805 \\
\hline 000617 & 100.28953 & 9.86389 & $\mathrm{~W}$ & 602095761 & QPS & 0.30 & 5.956 & $<1 \mathrm{E}-4$ & 0.15 & - \\
\hline 000619 & 100.31142 & 9.57033 & $\mathrm{c}$ & 603402479 & $\mathrm{D}$ & 0.69 & 6.404 & $5.0 \mathrm{E}-2$ & 0.57 & - \\
\hline 000620 & 100.33643 & 9.50333 & w & 602083907 & QPS & 0.36 & 8.6 & $<1 \mathrm{E}-4$ & 0.16 & - \\
\hline 000622 & 100.34081 & 9.75860 & $\mathrm{w}$ & 602091881 & QPS & 1.16 & 12.629 & $<1 \mathrm{E}-4$ & -0.05 & - \\
\hline 000624 & 100.26835 & 9.86390 & $\mathrm{w}$ & 616849454 & QPS & 1.85 & 7.47 & $8.0 \mathrm{E}-1^{b}$ & 0.50 & 7.5 \\
\hline 000629 & 100.27497 & 9.59762 & w & 616919784 & $\mathrm{U}$ & 1.20 & & & & - \\
\hline 000631 & 100.27462 & 9.38222 & & 223982068 & $\mathrm{~N}$ & & & & & - \\
\hline 000636 & 100.20351 & 9.72379 & $\mathrm{c}$ & 616872578 & $\mathrm{~S}$ & 0.62 & & & & - \\
\hline 000637 & 100.20504 & 9.96071 & $\mathrm{c}$ & 616826638 & $\mathrm{~L}$ & 0.45 & 12.306 & $<1 \mathrm{E}-4$ & & 12.5 \\
\hline 000638 & 100.26900 & 9.91213 & & 602266765 & $\mathrm{~N}$ & & & & & - \\
\hline 000646 & 100.19197 & 9.82152 & w & 616849567 & $\mathrm{P}$ & 2.72 & 0.746 & $<1 \mathrm{E}-4$ & 0.06 & 0.747 \\
\hline 000647 & 100.26233 & 9.79842 & $\mathrm{w}$ & 223981285 & MP & 1.41 & 1.181 & $<1 \mathrm{E}-4$ & & 1.152 \\
\hline 000647 & 100.26233 & 9.79842 & $\mathrm{w}$ & 223981285 & MP & 1.41 & 1.073 & $3.0 \mathrm{E}-3$ & & "" \\
\hline 000647 & 100.26233 & 9.79842 & W & 223981285 & MP & 1.41 & 1.119 & $3.0 \mathrm{E}-3$ & & "" \\
\hline 000647 & 100.26233 & 9.79842 & W & 223981285 & MP & 1.41 & 1.231 & $5.0 \mathrm{E}-3$ & & "" \\
\hline 000650 & 100.25409 & 9.54568 & $\mathrm{c}$ & 223980807 & $\mathrm{D}$ & 2.50 & & & & \\
\hline 000656 & 100.33554 & 9.79165 & $\mathrm{w}$ & 616872623 & $\mathrm{P}$ & 0.36 & 4.442 & $<1 \mathrm{E}-4$ & 0.14 & - \\
\hline 000657 & 100.32378 & 9.49060 & $\mathrm{~W}$ & 400007328 & QPS & 0.30 & 4.829 & $8.0 \mathrm{E}-1^{b}$ & 0.37 & 2.434 \\
\hline 000660 & 100.25215 & 9.48775 & $\mathrm{c}$ & 223980693 & QPD & 1.40 & 5.125 & $<1 \mathrm{E}-4$ & 0.34 & 5.282 \\
\hline 000667 & 100.36989 & 9.64413 & $\mathrm{c}$ & 223987997 & $\mathrm{D}$ & 1.53 & 5.897 & $1.0 \mathrm{E}-3$ & 0.62 & 6.456 \\
\hline 000676 & 100.44917 & 9.56935 & $\mathrm{c}$ & 223993199 & $\mathrm{D}$ & 1.20 & 9.404 & $<1 \mathrm{E}-4$ & 0.69 & \\
\hline 000677 & 100.24718 & 9.37275 & $\mathrm{~W}$ & 616969698 & $\mathrm{~N}$ & 0.23 & & & & - \\
\hline 000679 & 100.26681 & 9.39229 & & 223981550 & $\mathrm{U}$ & & & & & 14.58 \\
\hline 000680 & 100.30555 & 9.46872 & w & 603396406 & $\mathrm{P}$ & 1.09 & 5.768 & $<1 \mathrm{E}-4$ & 0.06 & - \\
\hline 000681 & 100.37965 & 9.44949 & $\mathrm{c}$ & 603808965 & $\mathrm{D}$ & 1.69 & & & & \\
\hline 000688 & 100.27123 & 9.81331 & W & 616849460 & QPS & 0.62 & 3.756 & $<1 \mathrm{E}-4$ & 0.02 & 3.748 \\
\hline 000689 & 100.21422 & 9.89006 & & 605538681 & $\mathrm{~N}$ & & & & & - \\
\hline 000695 & 100.38543 & 9.63537 & w & 223988965 & $\mathrm{P}$ & 0.90 & 3.235 & $<1 \mathrm{E}-4$ & -0.19 & 9.5 \\
\hline 000697 & 100.39398 & 9.60902 & $\mathrm{c}$ & 223989567 & $\mathrm{U}$ & 0.45 & & & & \\
\hline 000705 & 100.29283 & 9.55697 & $\mathrm{w}$ & 616919645 & MP? & 0.30 & 0.679 & $2.0 \mathrm{E}-2$ & & \\
\hline 000714 & 100.34228 & 9.35863 & w & 616969724 & & 0.30 & & & & 3.405 \\
\hline
\end{tabular}


Table F.1. continued.

\begin{tabular}{|c|c|c|c|c|c|c|c|c|c|c|}
\hline CSIMon-\# & $\mathrm{RA}^{1}$ & $\operatorname{Dec}^{1}$ & Class $^{2}$ & CoRoT_2011 & $\mathrm{LC}^{3}$ & $M_{\star}\left(M_{\odot}\right)$ & $P_{2011}^{\mathrm{CoRoT}}(\mathrm{d})$ & FAP $^{4}$ & $\mathrm{Q}^{5}$ & $P_{2008}^{\mathrm{CoRoT}}(\mathrm{d})^{6}$ \\
\hline 000717 & 100.31298 & 9.44565 & $\mathrm{c}$ & 616943877 & QPD & 0.53 & 8.558 & $<1 \mathrm{E}-4$ & 0.49 & - \\
\hline 000718 & 100.26733 & 9.34564 & $\mathrm{c}$ & 616969715 & $\mathrm{~N}$ & 0.34 & & & & - \\
\hline 000719 & 100.26848 & 9.85725 & $\mathrm{w}$ & 602095759 & QPS & 1.20 & 3.975 & $<1 \mathrm{E}-4$ & 0.28 & 4.024 \\
\hline 000723 & 100.21888 & 9.84961 & $\mathrm{w}$ & 616849436 & $\mathrm{U}$ & 0.32 & & & & - \\
\hline 000724 & 100.21947 & 9.73917 & w & 400007529 & QPS & 0.36 & 5.127 & $<1 \mathrm{E}-4$ & 0.18 & 4.842 \\
\hline 000728 & 100.28241 & 9.73417 & $\mathrm{w}$ & 223982535 & QPS & 1.58 & 5.158 & $<1 \mathrm{E}-4$ & 0.17 & 5.052 \\
\hline 000733 & 100.20749 & 9.79200 & $\mathrm{c}$ & 616872582 & $\mathrm{~N}$ & 0.25 & & & & - \\
\hline 000743 & 100.28086 & 9.51970 & $\mathrm{w}$ & 603402476 & $\mathrm{~N}$ & 0.32 & & & & - \\
\hline 000744 & 100.18384 & 9.83265 & & 616849558 & $\mathrm{~N}$ & & & & & - \\
\hline 000745 & 100.20112 & 9.61073 & $\mathrm{~W}$ & 603408576 & QPS & 0.45 & 1.669 & $<1 \mathrm{E}-4$ & 0.37 & - \\
\hline 000747 & 100.34176 & 9.72021 & $\mathrm{w}$ & 616872626 & $\mathrm{P}$ & 0.95 & 6.61 & $<1 \mathrm{E}-4$ & 0.00 & - \\
\hline 000749 & 100.30890 & 9.44460 & w & 616943875 & $\mathrm{P}$ & 0.45 & 1.436 & $<1 \mathrm{E}-4$ & 0.06 & - \\
\hline 000753 & 100.27486 & 9.65392 & $\mathrm{c}$ & 616895898 & $\mathrm{~N}$ & 0.21 & & & & 7.67 \\
\hline 000754 & 100.22094 & 9.88318 & $\mathrm{c}$ & 605538488 & $\mathrm{P}$ & 0.64 & 0.969 & $<1 \mathrm{E}-4$ & 0.13 & - \\
\hline 000755 & 100.19562 & 9.81333 & w & 605538556 & $\mathrm{Be}$ & 0.95 & 3.5 & $<1 \mathrm{E}-4$ & & - \\
\hline 000755 & 100.19562 & 9.81333 & $\mathrm{w}$ & 605538556 & $\mathrm{Be}$ & 0.95 & 4.054 & $<1 \mathrm{E}-4$ & & - \\
\hline 000756 & 100.18974 & 9.85641 & & 616849565 & $\mathrm{U}$ & & & & & - \\
\hline 000765 & 100.22349 & 9.55688 & $\mathrm{c}$ & 223978921 & QPS & 1.45 & 2.371 & $<1 \mathrm{E}-4$ & 0.22 & \\
\hline $000766^{8}$ & 100.20158 & 9.81069 & $\mathrm{c}$ & 602095741 & $\mathrm{~S}$ & 0.53 & 2.798 & $<1 \mathrm{E}-4$ & 0.63 & - \\
\hline 000770 & 100.31032 & 9.62065 & $\mathrm{w}$ & 616895918 & QPS & 0.66 & 5.442 & $<1 \mathrm{E}-4$ & -0.05 & 5.405 \\
\hline 000771 & 100.32610 & 9.56489 & $\mathrm{c}$ & 223985261 & U & 1.38 & & & & 18.08 \\
\hline $000774^{*}$ & 100.24519 & 9.51592 & $\mathrm{c}$ & 223980264 & S & 1.83 & 3.49 & $2.0 \mathrm{E}-4$ & 0.82 & 3.482 \\
\hline 000777 & 100.26328 & 9.43417 & w & 400007811 & $\mathrm{U}$ & 0.67 & & & & - \\
\hline 000779* & 100.20080 & 9.45026 & w & 602079840 & $\mathrm{U}$ & 0.21 & 3.131 & $1.0 \mathrm{E}-3$ & 1.27 & - \\
\hline 000784 & 100.25591 & 9.56895 & w & 616919771 & $\mathrm{P}$ & 1.20 & 10.098 & $<1 \mathrm{E}-4$ & -0.01 & - \\
\hline 000794 & 100.30442 & 9.38455 & $\mathrm{w}$ & 616969735 & & 0.47 & 4.031 & $2.0 \mathrm{E}-1^{a}$ & - & - \\
\hline 000797 & 100.20071 & 9.40329 & & 603808962 & $\mathrm{~N}$ & & & & & - \\
\hline 000798 & 100.23731 & 9.81134 & $\mathrm{w}$ & 223979759 & QPS & 1.57 & 3.808 & $<1 \mathrm{E}-4$ & -0.33 & 3.84 \\
\hline 000804 & 100.23216 & 9.85385 & $\mathrm{c}$ & 616849440 & B & 1.06 & 3.271 & $<1 \mathrm{E}-4$ & 0.41 & 3.217 \\
\hline 000805 & 100.43724 & 9.74455 & $\mathrm{w}$ & 223992383 & & 0.71 & 3.425 & $9.0 \mathrm{E}-3$ & 0.51 & 3.38 \\
\hline 000808 & 100.21494 & 9.47905 & $\mathrm{c}$ & 603396401 & B & 1.24 & & & & - \\
\hline 000809 & 100.26456 & 9.52181 & w & 223981406 & $\mathrm{P}$ & 1.58 & 2.167 & $<1 \mathrm{E}-4$ & 0.09 & 2.157 \\
\hline 000810 & 100.29096 & 9.45339 & $\mathrm{c}$ & 605538241 & $\mathrm{P}$ & 1.20 & 2.925 & $<1 \mathrm{E}-4$ & 0.06 & 2.914 \\
\hline 000811 & 100.18004 & 9.78534 & $\mathrm{c}$ & 605538574 & QPD & 0.91 & 7.844 & $<1 \mathrm{E}-4$ & 0.41 & 8.49 \\
\hline 000819 & 100.37159 & 9.65997 & w & 223988099 & QPS & 1.65 & 3.333 & $<1 \mathrm{E}-4$ & 0.23 & 3.273 \\
\hline 000823 & 100.20889 & 9.95111 & $\mathrm{c}$ & 603809233 & U & 0.30 & & & & - \\
\hline 000826 & 100.21700 & 9.87052 & $\mathrm{w}$ & 616849435 & $\mathrm{U}$ & 0.67 & 14.531 & $<1 \mathrm{E}-4$ & 0.55 & - \\
\hline $000842^{\#}$ & 100.24214 & 9.85375 & $\mathrm{~W}$ & 605538526 & MP & 0.33 & 11.094 & $<1 \mathrm{E}-4$ & & - \\
\hline $000842^{\#}$ & 100.24214 & 9.85375 & $\mathrm{w}$ & 605538526 & MP & 0.33 & 1.917 & $2.0 \mathrm{E}-4$ & & - \\
\hline 000843 & 100.21404 & 9.50371 & $\mathrm{w}$ & 400007916 & $\mathrm{~N}$ & 0.21 & & & & - \\
\hline 000848 & 100.35306 & 9.43983 & $\mathrm{w}$ & 223986923 & QPS & 1.35 & 8.506 & $<1 \mathrm{E}-4$ & -0.14 & 8.3 \\
\hline 000869 & 100.23942 & 9.48981 & w & 603808963 & $\mathrm{P}$ & 0.32 & 8.898 & $<1 \mathrm{E}-4$ & 0.42 & 8.854 \\
\hline 000870 & 100.20729 & 9.88296 & & 616849427 & $\mathrm{U}$ & 0.30 & & & & - \\
\hline 000872 & 100.19208 & 9.79727 & $\mathrm{w}$ & 602266744 & QPS & 0.36 & 5.927 & $<1 \mathrm{E}-4$ & 1.36 & - \\
\hline 000876 & 100.30052 & 9.49774 & $\mathrm{w}$ & 616944031 & $\mathrm{U}$ & 0.21 & & & & - \\
\hline 000877 & 100.31993 & 9.45839 & $\mathrm{c}$ & 616943883 & $\mathrm{~B}$ & 1.33 & 5.177 & $<1 \mathrm{E}-4$ & 0.50 & - \\
\hline 000878 & 100.34572 & 9.49187 & $\mathrm{w}$ & 616943892 & $\mathrm{~N}$ & 0.28 & & & & - \\
\hline 000879 & 100.26409 & 9.67912 & $\mathrm{c}$ & 603408580 & $\mathrm{~S}$ & 0.45 & 11.398 & $<1 \mathrm{E}-4$ & 0.25 & - \\
\hline 000881 & 100.28715 & 9.68745 & $\mathrm{w}$ & 616895909 & $\mathrm{P}$ & 1.16 & 3.919 & $<1 \mathrm{E}-4$ & 0.18 & - \\
\hline 000886 & 100.18767 & 9.76161 & W & 616872573 & $\mathrm{P}$ & 0.71 & 4.612 & $<1 \mathrm{E}-4$ & 0.05 & 4.625 \\
\hline 000890 & 100.22993 & 9.84716 & W & 616849420 & $\mathrm{P}$ & 1.05 & 1.165 & $<1 \mathrm{E}-4$ & 0.05 & 1.165 \\
\hline 000892 & 100.25646 & 9.47031 & & 223980955 & QPS & 2.26 & 2.415 & $1.0 \mathrm{E}-2$ & 0.54 & - \\
\hline 000894 & 100.31422 & 9.77766 & $\mathrm{w}$ & 223984520 & $\mathrm{P}$ & 1.60 & 1.463 & $<1 \mathrm{E}-4$ & 0.14 & 1.469 \\
\hline 000901 & 100.18062 & 9.84986 & $\mathrm{w}$ & 616849547 & QPS & 0.69 & 9.031 & $<1 \mathrm{E}-4$ & 0.21 & 9.114 \\
\hline 000906 & 100.27711 & 9.89594 & & 605538675 & $\mathrm{~N}$ & 0.37 & & & & - \\
\hline 000907 & 100.39952 & 9.67830 & $\mathrm{w}$ & 616895801 & $\mathrm{~N}$ & 0.30 & & & & - \\
\hline 000910 & 100.19965 & 9.55087 & $\mathrm{w}$ & 603809014 & QPS & 0.31 & 2.581 & $<1 \mathrm{E}-4$ & 0.57 & 2.568 \\
\hline 000913 & 100.24680 & 9.88552 & & 605538690 & $\mathrm{U}$ & & & & & - \\
\hline
\end{tabular}


Table F.1. continued.

\begin{tabular}{|c|c|c|c|c|c|c|c|c|c|c|}
\hline CSIMon-\# & $\mathrm{RA}^{1}$ & $\operatorname{Dec}^{1}$ & Class $^{2}$ & CoRoT_2011 & $\mathrm{LC}^{3}$ & $M_{\star}\left(M_{\odot}\right)$ & $P_{2011}^{\mathrm{CoRoT}}(\mathrm{d})$ & FAP $^{4}$ & $\mathrm{Q}^{5}$ & $P_{2008}^{\mathrm{CoRoT}}(\mathrm{d})^{6}$ \\
\hline 000914 & 100.21629 & 9.63219 & $\mathrm{c}$ & 602087948 & $\mathrm{U}$ & & & & & - \\
\hline 000919 & 100.30541 & 9.53064 & $\mathrm{c}$ & 616919654 & B & 0.25 & & & & - \\
\hline 000923 & 100.42167 & 9.54519 & & 223991355 & $\mathrm{U}$ & & & & & \\
\hline 000925 & 100.19408 & 9.36140 & & 223977092 & $\mathrm{U}$ & & & & & \\
\hline 000926 & 100.27678 & 9.47746 & $\mathrm{c}$ & 400007687 & QPS & 0.40 & 12.323 & $<1 \mathrm{E}-4$ & - & \\
\hline 000927 & 100.41256 & 9.55449 & w & 616919568 & QPS & 0.25 & 5.912 & $<1 \mathrm{E}-4$ & 0.05 & - \\
\hline 000928 & 100.35674 & 9.57862 & $\mathrm{c}$ & 223987178 & $\mathrm{~N}$ & 0.63 & & & & 9.84 \\
\hline 000931 & 100.32534 & 9.64042 & $\mathrm{c}$ & 616895920 & $\mathrm{~N}$ & 0.29 & & & & \\
\hline 000932 & 100.18689 & 9.96229 & w & 223976672 & $\mathrm{P}$ & 0.90 & 15.373 & $<1 \mathrm{E}-4$ & 0.09 & 15.0 \\
\hline 000936 & 100.27988 & 9.45816 & $\mathrm{c}$ & 605538236 & B & 1.20 & & & & - \\
\hline 000937 & 100.21896 & 9.86833 & $\mathrm{c}$ & 603809175 & $\mathrm{~N}$ & 0.69 & & & & \\
\hline 000938 & 100.17636 & 9.57362 & $\mathrm{~W}$ & 616919726 & QP & 0.29 & 7.037 & $1.0 \mathrm{E}-3$ & 0.44 & - \\
\hline 000941 & 100.36975 & 9.45299 & w & 400007743 & $\mathrm{P}$ & 0.30 & 1.313 & $<1 \mathrm{E}-4$ & 0.61 & - \\
\hline 000945 & 100.20787 & 9.61375 & $\mathrm{c}$ & 223977953 & B & 1.40 & & & & 4.919 \\
\hline 000948 & 100.36316 & 9.58504 & w & 223987553 & QPS & 1.49 & 1.546 & $<1 \mathrm{E}-4$ & 0.08 & 1.544 \\
\hline 000949 & 100.29927 & 9.39239 & & 616969729 & $\mathrm{~N}$ & 0.31 & & & & - \\
\hline 000951 & 100.32468 & 9.48365 & $\mathrm{c}$ & 602079852 & QP & 0.33 & 2.913 & $1.0 \mathrm{E}-4$ & 0.61 & 10.44 \\
\hline 000954 & 100.28063 & 9.43196 & $\mathrm{~W}$ & 603396405 & $\mathrm{P}$ & 1.40 & 7.352 & $<1 \mathrm{E}-4$ & 0.04 & - \\
\hline 000958 & 100.23104 & 9.15800 & w & 603808801 & & 0.42 & 0.712 & $1.7 \mathrm{E}-1^{d}$ & - & - \\
\hline 000964 & 100.27966 & 9.21065 & $\mathrm{c}$ & 223982375 & QPS & 0.95 & 3.289 & $<1 \mathrm{E}-4$ & 0.54 & 3.32 \\
\hline 000965 & 100.19170 & 9.29947 & $\mathrm{c}$ & 616996507 & QPS & 0.36 & 9.688 & $<1 \mathrm{E}-4$ & 0.01 & 9.786 \\
\hline 000967 & 100.30676 & 9.23151 & W & 223984026 & $\mathrm{Be}$ & 1.20 & 3.352 & $<1 \mathrm{E}-4$ & & - \\
\hline 000967 & 100.30676 & 9.23151 & w & 223984026 & $\mathrm{Be}$ & 1.20 & 2.781 & $<1 \mathrm{E}-4$ & & - \\
\hline 000977 & 100.24625 & 9.28319 & w & 616996570 & $\mathrm{P}$ & 0.25 & 0.719 & $<1 \mathrm{E}-4$ & 0.66 & - \\
\hline 000981 & 100.29208 & 9.24239 & & 223983112 & $\mathrm{U}$ & & & & & - \\
\hline 000985 & 100.06316 & 10.03274 & $\mathrm{c}$ & 223969098 & $\mathrm{D}$ & 0.90 & & & & \\
\hline 000989 & 100.03549 & 9.73707 & $\mathrm{w}$ & 223967301 & $\mathrm{P}$ & 0.45 & 0.958 & $<1 \mathrm{E}-4$ & 0.14 & 0.957 \\
\hline 000990 & 100.15702 & 9.66106 & w & 616896002 & $\mathrm{~N}$ & 0.75 & & & & - \\
\hline 000991 & 100.08045 & 9.80829 & w & 616849658 & $\mathrm{P}$ & 0.62 & 1.033 & $<1 \mathrm{E}-4$ & 0.06 & - \\
\hline 000995 & 100.10707 & 9.97660 & w & 223971866 & QPS & 1.75 & 9.284 & $1.0 \mathrm{E}-4$ & 0.55 & 7.015 \\
\hline 000996 & 100.17216 & 9.85068 & $\mathrm{c}$ & 616849542 & S & 0.69 & & & & 7.812 \\
\hline 001000 & 100.04867 & 9.76557 & $\mathrm{w}$ & 602091887 & $\mathrm{P}$ & 0.36 & 1.433 & $<1 \mathrm{E}-4$ & 0.31 & - \\
\hline 001001 & 99.98408 & 9.51282 & & 223963815 & MP & & 2.971 & $<1 \mathrm{E}-4$ & & \\
\hline 001001 & 99.98408 & 9.51282 & & 223963815 & MP & & 2.706 & $<1 \mathrm{E}-4$ & & \\
\hline 001001 & 99.98408 & 9.51282 & & 223963815 & MP & & 3.269 & $7.0 \mathrm{E}-3$ & & \\
\hline 001002 & 100.10938 & 9.63374 & w & 223971984 & $\mathrm{P}$ & 0.61 & 6.298 & $<1 \mathrm{E}-4$ & 0.06 & 6.281 \\
\hline 001003 & 100.10686 & 9.99994 & $\mathrm{c}$ & 616803611 & $\mathrm{U}$ & 0.24 & 3.454 & $2.0 \mathrm{E}-4$ & 0.55 & 3.469 \\
\hline 001005 & 100.02174 & 9.84904 & $\mathrm{~W}$ & 616849610 & QPS & 0.33 & 1.769 & $<1 \mathrm{E}-4$ & 0.45 & - \\
\hline 001009 & 99.94280 & 9.80551 & w & 223960995 & $\mathrm{~N}$ & 0.65 & & & & \\
\hline 001012 & 100.13880 & 9.98137 & w & 223973818 & $\mathrm{U}$ & 0.65 & 4.465 & $<1 \mathrm{E}-4$ & 2.21 & - \\
\hline 001015 & 99.91381 & 9.93322 & w & 223958963 & MP & 0.62 & 4.246 & $7.0 \mathrm{E}-1^{b}$ & & 0.859 \\
\hline 001015 & 99.91381 & 9.93322 & w & 223958963 & MP & 0.62 & 0.858 & $<1 \mathrm{E}-4$ & & "" \\
\hline 001016 & 100.08762 & 9.60888 & w & 223970694 & $\mathrm{P}$ & 1.67 & 1.483 & $<1 \mathrm{E}-4$ & 0.27 & 1.467 \\
\hline 001017 & 100.09888 & 9.92329 & $\mathrm{c}$ & 223971383 & B & 0.30 & & & & 4.648 \\
\hline 001022 & 100.16297 & 9.84962 & $\mathrm{c}$ & 616849543 & B & 1.50 & & & & 13.88 \\
\hline $001023^{\#}$ & 100.04293 & 9.64862 & w & 223967803 & MP & 0.69 & 3.827 & $<1 \mathrm{E}-4$ & & 3.841 \\
\hline $001023^{\#}$ & 100.04293 & 9.64862 & w & 223967803 & MP & 0.69 & 0.629 & $<1 \mathrm{E}-4$ & & "" \\
\hline 001027 & 100.15500 & 9.51941 & w & 616919872 & $\mathrm{P}$ & 0.33 & 7.217 & $<1 \mathrm{E}-4$ & 0.04 & - \\
\hline 001031 & 100.16687 & 9.58415 & $\mathrm{c}$ & 616919878 & QPS & 1.46 & 4.448 & $<1 \mathrm{E}-4$ & 0.42 & - \\
\hline 001037 & 100.12858 & 9.57792 & $\mathrm{c}$ & 223973200 & QPS & 1.45 & 8.877 & $<1 \mathrm{E}-4$ & 0.55 & \\
\hline 001038 & 100.09427 & 9.82952 & $\mathrm{c}$ & 602095739 & $\mathrm{D}$ & 0.66 & 6.383 & $5.0 \mathrm{E}-3$ & 0.62 & - \\
\hline 001047 & 99.98171 & 9.79216 & w & 223963678 & MP & 0.30 & 0.677 & $<1 \mathrm{E}-4$ & & 0.676 \\
\hline 001047 & 99.98171 & 9.79216 & w & 223963678 & MP & 0.30 & 0.914 & $2.0 \mathrm{E}-3$ & & "" \\
\hline 001048 & 100.01110 & 9.59007 & $\mathrm{c}$ & 616919938 & $\mathrm{~B}$ & 0.30 & & & & - \\
\hline 001053 & 100.17140 & 9.88236 & $\mathrm{c}$ & 602095745 & $\mathrm{~S}$ & 0.91 & 11.838 & $<1 \mathrm{E}-4$ & 0.52 & - \\
\hline 001054 & 100.15217 & 9.84600 & $\mathrm{c}$ & 400007538 & $\mathrm{~S}$ & 0.36 & 8.142 & $2.0 \mathrm{E}-2^{c}$ & 0.49 & \\
\hline 001055 & 100.16845 & 9.84734 & $\mathrm{c}$ & 616849545 & QPS & 0.68 & 3.731 & $<1 \mathrm{E}-4$ & 0.23 & 3.748 \\
\hline 001056 & 100.02624 & 9.59904 & w & 616919952 & $\mathrm{P}$ & 0.36 & 1.519 & $5.0 \mathrm{E}-1^{d}$ & 0.44 & - \\
\hline
\end{tabular}


Table F.1. continued.

\begin{tabular}{|c|c|c|c|c|c|c|c|c|c|c|}
\hline CSIMon-\# & $\mathrm{RA}^{1}$ & $\operatorname{Dec}^{1}$ & Class $^{2}$ & CoRoT_2011 & $\mathrm{LC}^{3}$ & $M_{\star}\left(M_{\odot}\right)$ & $P_{2011}^{\text {CoRoT }}(\mathrm{d})$ & $\mathrm{FAP}^{4}$ & $\mathrm{Q}^{5}$ & $P_{2008}^{\text {CoRoT }}(\mathrm{d})^{6}$ \\
\hline 001059 & 100.08598 & 9.68055 & $\mathrm{c}$ & 616895951 & QPS & 0.22 & 5.819 & $<1 \mathrm{E}-4$ & 0.20 & - \\
\hline 001061 & 100.10065 & 9.57013 & $\mathrm{c}$ & 616919835 & $\mathrm{~S}$ & 0.64 & & & & - \\
\hline 001062 & 100.10348 & 9.88652 & & 616849505 & $\mathrm{U}$ & & & & & - \\
\hline 001064 & 100.14658 & 9.86579 & $\mathrm{c}$ & 616849538 & $\mathrm{P}$ & 0.45 & 2.698 & $<1 \mathrm{E}-4$ & -0.06 & - \\
\hline 001067 & 100.04552 & 9.90725 & $\mathrm{~W}$ & 602099690 & MP? & 0.29 & 1.148 & $6.0 \mathrm{E}-4$ & & - \\
\hline 001072 & 100.13061 & 9.69147 & w & 223973318 & $\mathrm{~N}$ & 1.01 & & & & \\
\hline 001074 & 100.03512 & 9.99052 & W & 616826786 & & 0.28 & & & & - \\
\hline 001076 & 100.15916 & 9.49792 & $\mathrm{c}$ & 605424384 & $\mathrm{U}$ & 0.45 & & & & 5.884 \\
\hline 001077 & 100.17760 & 9.89659 & & 616849552 & QPS & 1.00 & 8.746 & $<1 \mathrm{E}-4$ & -0.05 & - \\
\hline 001081 & 100.08732 & 9.39841 & w & 223970675 & $\mathrm{~N}$ & 0.76 & & & & - \\
\hline 001082 & 100.00467 & 9.59265 & w & 616919920 & QPS & 0.33 & 9.185 & $<1 \mathrm{E}-4$ & - & 9.114 \\
\hline 001085 & 100.13667 & 9.85815 & $\mathrm{~W}$ & 223973692 & $\mathrm{P}$ & 0.91 & 3.452 & $<1 \mathrm{E}-4$ & 0.09 & 3.456 \\
\hline 001087 & 100.00504 & 9.71013 & w & 616872743 & & 0.30 & 3.931 & $1.0 \mathrm{E}-2$ & & - \\
\hline 001089 & 100.12452 & 9.83622 & W & 223972960 & $\mathrm{U}$ & 1.55 & & & & \\
\hline 001092 & 100.16125 & 9.61581 & w & 602087946 & QPS & 0.30 & 1.317 & $<1 \mathrm{E}-4$ & - & - \\
\hline 001094 & 100.13183 & 9.80649 & $\mathrm{c}$ & 603420177 & QPS & 1.14 & 4.246 & $<1 \mathrm{E}-4$ & 0.39 & - \\
\hline 001099 & 100.17235 & 9.90385 & $\mathrm{c}$ & 223975844 & QPS & 1.52 & 3.419 & $<1 \mathrm{E}-4$ & 0.36 & 3.332 \\
\hline 001100 & 100.16393 & 9.57930 & $\mathrm{c}$ & 616919877 & $\mathrm{U}$ & 0.90 & & & & - \\
\hline 001101 & 100.04533 & 9.64467 & W & 603408567 & $\mathrm{U}$ & 0.53 & & & & - \\
\hline 001102 & 100.05041 & 9.82508 & $\mathrm{w}$ & 602095736 & $\mathrm{U}$ & 1.20 & & & & - \\
\hline 001105 & 100.12026 & 9.55154 & W & 616919856 & $\mathrm{Be}$ & 0.30 & 0.758 & $<1 \mathrm{E}-4$ & & - \\
\hline 001105 & 100.12026 & 9.55154 & w & 616919856 & $\mathrm{Be}$ & 0.30 & 0.958 & $<1 \mathrm{E}-4$ & & - \\
\hline 001114 & 99.88913 & 9.86714 & $\mathrm{c}$ & 223957142 & QPS & 0.40 & 2.579 & $<1 \mathrm{E}-4$ & 0.41 & 2.568 \\
\hline 001115 & 100.00896 & 9.75395 & $\mathrm{w}$ & 223965459 & $\mathrm{P}$ & 0.83 & 1.352 & $<1 \mathrm{E}-4$ & 0.03 & 1.351 \\
\hline 001126 & 100.04007 & 9.69540 & w & 223967602 & & 1.62 & 1.233 & $<1 \mathrm{E}-4$ & 0.19 & 1.236 \\
\hline 001131 & 99.89339 & 9.91422 & $\mathrm{c}$ & 223957455 & QPD & 0.36 & 5.144 & $<1 \mathrm{E}-4$ & 0.44 & 10.16 \\
\hline 001132 & 100.10781 & 9.84933 & $\mathrm{c}$ & 602095740 & QPS & 0.33 & 2.958 & $<1 \mathrm{E}-4$ & 0.51 & - \\
\hline 001133 & 99.99820 & 9.94008 & $\mathrm{w}$ & 223964746 & $\mathrm{P}$ & 0.95 & 1.25 & $<1 \mathrm{E}-4$ & 0.10 & - \\
\hline 001140 & 99.92278 & 9.77213 & $\mathrm{c}$ & 223959618 & QPD & 1.31 & 3.917 & $<1 \mathrm{E}-4$ & 0.48 & 3.922 \\
\hline 001142 & 100.14361 & 9.58839 & W & 616919870 & $\mathrm{~N}$ & 0.36 & & & & - \\
\hline 001144 & 100.09620 & 9.46176 & $\mathrm{c}$ & 223971231 & QP & 1.12 & 4.106 & $1.0 \mathrm{E}-4$ & 0.75 & \\
\hline 001147 & 99.95761 & 9.93939 & $\mathrm{~W}$ & 223962024 & $\mathrm{~N}$ & 0.88 & & & & \\
\hline 001149 & 100.12741 & 9.83736 & $\mathrm{c}$ & 603420176 & $\mathrm{~N}$ & 0.30 & & & & - \\
\hline 001152 & 100.16453 & 9.81102 & $\mathrm{~W}$ & 605539518 & $\mathrm{U}$ & 1.30 & & & & - \\
\hline 001156 & 100.13984 & 9.56013 & $\mathrm{c}$ & 616919866 & $\mathrm{U}$ & 0.30 & 16.335 & $<1 \mathrm{E}-4$ & 0.24 & - \\
\hline 001157 & 100.08369 & 9.47460 & $\mathrm{c}$ & 223970440 & QPS & 0.95 & 3.813 & $<1 \mathrm{E}-4$ & 0.43 & \\
\hline 001158 & 99.85939 & 9.68634 & $\mathrm{~W}$ & 223955032 & QPS & 0.62 & 5.546 & $<1 \mathrm{E}-4$ & -0.06 & 5.436 \\
\hline 001163 & 100.12398 & 9.99260 & w & 223972918 & $\mathrm{U}$ & 0.65 & & & & \\
\hline 001167 & 100.15781 & 9.58166 & $\mathrm{c}$ & 400007528 & QPS & 0.30 & 8.804 & $<1 \mathrm{E}-4$ & 0.22 & 9.42 \\
\hline 001171 & 99.89160 & 9.82245 & $\mathrm{c}$ & 223957322 & $\mathrm{D}$ & 0.70 & & & & 18.05 \\
\hline 001172 & 100.02658 & 9.65932 & $\mathrm{~W}$ & 616896077 & QPS & 0.65 & 6.492 & $<1 \mathrm{E}-4$ & 0.06 & - \\
\hline 001174 & 100.05711 & 9.94183 & $\mathrm{c}$ & 616826810 & B & 0.35 & 6.84 & $4.0 \mathrm{E}-3$ & 0.80 & \\
\hline 001181 & 100.07507 & 9.83947 & $\mathrm{~W}$ & 616849652 & QPS & 0.35 & 6.1 & $<1 \mathrm{E}-4$ & 0.64 & - \\
\hline 001187 & 100.05904 & 9.57453 & $\mathrm{c}$ & 602083884 & B & 0.40 & 3.102 & $3.0 \mathrm{E}-4$ & 0.86 & - \\
\hline 001189 & 100.16258 & 9.60000 & $\mathrm{w}$ & 223975253 & $\mathrm{P}$ & 0.63 & 4.581 & $<1 \mathrm{E}-4$ & 0.55 & \\
\hline 001193 & 99.89478 & 9.78170 & $\mathrm{~W}$ & 602091877 & $\mathrm{U}$ & 0.66 & & & & - \\
\hline 001194 & 100.15287 & 9.36811 & w & 223974689 & $\mathrm{~N}$ & 0.65 & & & & \\
\hline 001195 & 100.14539 & 9.90199 & W & 400007919 & $\mathrm{U}$ & 0.25 & & & & \\
\hline 001196 & 99.98682 & 9.74033 & & 223963994 & $\mathrm{U}$ & & & & & \\
\hline 001197 & 100.10900 & 9.61923 & $\mathrm{c}$ & 603408572 & QPS & 0.30 & 4.027 & $<1 \mathrm{E}-4$ & 0.50 & - \\
\hline 001199 & 100.17434 & 9.86241 & $\mathrm{c}$ & 602095746 & QPS & 1.20 & 3.617 & $<1 \mathrm{E}-4$ & 0.57 & 3.674 \\
\hline 001200 & 100.05182 & 9.73973 & $\mathrm{w}$ & 223968398 & $\mathrm{P}$ & 0.45 & 2.694 & $<1 \mathrm{E}-4$ & 0.38 & 2.702 \\
\hline 001201 & 100.15263 & 9.80638 & $\mathrm{~W}$ & 605539512 & $\mathrm{P}$ & 1.16 & 16.435 & $<1 \mathrm{E}-4$ & $<0$ & 15.25 \\
\hline 001204 & 99.95670 & 9.55611 & $\mathrm{~W}$ & 223961941 & QPS & 1.12 & 6.552 & $<1 \mathrm{E}-4$ & 0.04 & 6.52 \\
\hline 001205 & 100.12003 & 9.80664 & w & 400007344 & $\mathrm{P}$ & 1.13 & 6.861 & $<1 \mathrm{E}-4$ & 0.02 & - \\
\hline 001209 & 100.00597 & 9.51826 & $\mathrm{w}$ & 223965280 & $\mathrm{~N}$ & 0.64 & & & & \\
\hline
\end{tabular}


Table F.1. continued.

\begin{tabular}{|c|c|c|c|c|c|c|c|c|c|c|}
\hline CSIMon-\# & $\mathrm{RA}^{1}$ & $\operatorname{Dec}^{1}$ & Class $^{2}$ & CoRoT_2011 & $\mathrm{LC}^{3}$ & $M_{\star}\left(M_{\odot}\right)$ & $P_{2011}^{\mathrm{CoRoT}}(\mathrm{d})$ & FAP $^{4}$ & $\mathrm{Q}^{5}$ & $P_{2008}^{\text {CoRoT }}(\mathrm{d})^{6}$ \\
\hline 001217 & 100.15274 & 9.86756 & $\mathrm{c}$ & 616849540 & $\mathrm{~B}$ & 1.30 & 7.865 & $<1 \mathrm{E}-4$ & 0.59 & - \\
\hline 001218 & 100.11910 & 9.59657 & $\mathrm{~W}$ & 616919855 & $\mathrm{U}$ & 0.62 & 4.592 & $<1 \mathrm{E}-4$ & - & - \\
\hline 001219 & 100.11435 & 9.87510 & $\mathrm{c}$ & 616849516 & $\mathrm{~N}$ & 0.23 & & & & - \\
\hline 001221 & 100.09760 & 9.91543 & $\mathrm{c}$ & 616826682 & & 0.22 & 8.221 & $<1 \mathrm{E}-4$ & 0.56 & - \\
\hline 001222 & 100.17577 & 9.81426 & & 605538554 & $\mathrm{U}$ & & & & & - \\
\hline 001223 & 100.02502 & 9.82851 & $\mathrm{c}$ & 616849613 & QPS & 0.36 & 8.113 & $<1 \mathrm{E}-4$ & 0.27 & - \\
\hline 001226 & 100.06400 & 9.71178 & W & 602091889 & $\mathrm{~N}$ & 0.70 & & & & - \\
\hline 001232 & 100.15773 & 9.83083 & w & 602095744 & $\mathrm{U}$ & 0.31 & & & & - \\
\hline 001234 & 100.04634 & 9.63499 & $\mathrm{c}$ & 223968039 & QP & 0.94 & 9.606 & $<1 \mathrm{E}-4$ & 0.62 & \\
\hline $001236^{\#}$ & 100.09258 & 9.90801 & w & 223971008 & MP & 0.61 & 7.204 & $<1 \mathrm{E}-4$ & & 7.38 \\
\hline $001236^{\#}$ & 100.09258 & 9.90801 & w & 223971008 & MP & 0.61 & 2.396 & $<1 \mathrm{E}-4$ & & "" \\
\hline 001238 & 99.96290 & 9.60913 & w & 616896186 & $\mathrm{P}$ & 0.43 & 1.363 & $<1 \mathrm{E}-4$ & 0.08 & - \\
\hline 001239 & 99.91183 & 9.86440 & w & 223958794 & $\mathrm{~N}$ & 1.47 & & & & \\
\hline 001240 & 100.10996 & 9.94970 & $\mathrm{c}$ & 616826701 & & & 1.792 & $2.0 \mathrm{E}-2$ & - & - \\
\hline 001247 & 100.12060 & 9.70475 & $\mathrm{w}$ & 223972691 & QPS & 0.93 & 7.344 & $<1 \mathrm{E}-4$ & 0.28 & 7.206 \\
\hline 001248 & 99.94468 & 9.68164 & w & 223961132 & $\mathrm{P}$ & 1.39 & 3.842 & $<1 \mathrm{E}-4$ & 0.02 & 3.839 \\
\hline 001249 & 100.08446 & 9.93510 & $\mathrm{c}$ & 616826670 & QP & 0.30 & 1.954 & $<1 \mathrm{E}-4$ & 0.42 & - \\
\hline 001250 & 100.08611 & 9.57623 & w & 603402460 & QPS & 0.75 & 2.385 & $<1 \mathrm{E}-4$ & 0.60 & - \\
\hline 001251 & 100.13262 & 9.60018 & w & 616895982 & $\mathrm{U}$ & 0.33 & & & & - \\
\hline 001254 & 100.07084 & 9.77590 & w & 602091890 & $\mathrm{P}$ & 0.63 & 0.779 & $<1 \mathrm{E}-4$ & 0.11 & - \\
\hline 001256 & 99.92321 & 9.57794 & $\mathrm{w}$ & 223959652 & $\mathrm{P}$ & 1.36 & 3.65 & $<1 \mathrm{E}-4$ & 0.10 & 3.732 \\
\hline 001259 & 100.14511 & 9.76257 & $\mathrm{w}$ & 616872718 & $\mathrm{~N}$ & 0.32 & & & & - \\
\hline $001261^{11}$ & 100.07197 & 9.42894 & w & 602079822 & & 0.25 & & & & - \\
\hline 001264 & 100.12756 & 9.76961 & w & 602091893 & $\mathrm{P}$ & 1.32 & 7.171 & $<1 \mathrm{E}-4$ & -0.12 & 7.151 \\
\hline 001265 & 100.04535 & 9.66871 & w & 616896095 & $\mathrm{U}$ & 0.28 & 12.261 & $<1 \mathrm{E}-4$ & 1.43 & - \\
\hline 001271 & 100.01113 & 9.69690 & w & 616896061 & $\mathrm{~N}$ & 0.32 & & & & 10.0 \\
\hline 001274 & 100.17260 & 9.80267 & w & 605539508 & $\mathrm{P}$ & 1.20 & 4.712 & $<1 \mathrm{E}-4$ & 0.08 & 4.743 \\
\hline 001275 & 100.11978 & 9.51669 & $\mathrm{c}$ & 223972652 & $\mathrm{~S}$ & 1.60 & & & & \\
\hline 001277 & 100.11050 & 9.58946 & w & 602083887 & & 0.44 & 12.206 & $<1 \mathrm{E}-4$ & 4.99 & - \\
\hline 001278 & 100.17415 & 9.83120 & w & 616849549 & $\mathrm{P}$ & 0.63 & 1.05 & $<1 \mathrm{E}-4$ & 0.41 & 1.049 \\
\hline 001279 & 100.13016 & 9.51862 & w & 223973292 & $\mathrm{P}$ & 0.91 & 1.975 & $<1 \mathrm{E}-4$ & 0.04 & 1.974 \\
\hline 001281 & 99.84457 & 9.28438 & $\mathrm{w}$ & 223953966 & MP & 1.60 & 4.132 & $<1 \mathrm{E}-4$ & & 3.987 \\
\hline 001281 & 99.84457 & 9.28438 & $\mathrm{w}$ & 223953966 & MP & 1.60 & 0.254 & $5.0 \mathrm{E}-1^{d}$ & & "" \\
\hline 001281 & 99.84457 & 9.28438 & $\mathrm{w}$ & 223953966 & MP & 1.60 & 0.084 & & & "" \\
\hline 001284 & 100.05605 & 9.32427 & w & 223968646 & $\mathrm{~N}$ & 1.10 & & & & \\
\hline 001286 & 100.04946 & 9.35282 & w & 223968235 & $\mathrm{P}$ & 0.45 & 5.423 & $<1 \mathrm{E}-4$ & 0.11 & - \\
\hline 001290 & 99.87659 & 9.56040 & w & 223956264 & $\mathrm{P}$ & 0.70 & 2.248 & $<1 \mathrm{E}-4$ & 0.10 & 2.229 \\
\hline 001291 & 99.96803 & 9.31930 & W & 223962712 & $\mathrm{~N}$ & 0.45 & & & & \\
\hline 001292 & 99.94881 & 9.43520 & w & 223961409 & MP & 1.48 & 1.025 & $9.0 \mathrm{E}-3$ & 1.07 & 1.104 \\
\hline 001292 & 99.94881 & 9.43520 & w & 223961409 & MP & 1.48 & 1.15 & $1.0 \mathrm{E}-2$ & & "" \\
\hline 001294 & 100.02303 & 9.37390 & $\mathrm{c}$ & 616970063 & QP & 0.92 & 6.723 & $1.0 \mathrm{E}-4$ & 0.48 & - \\
\hline 001295 & 100.06584 & 9.35924 & w & 602075331 & $\mathrm{P}$ & 0.45 & 0.643 & $<1 \mathrm{E}-4$ & 0.45 & - \\
\hline 001296 & 99.76562 & 9.67317 & $\mathrm{c}$ & 223948127 & QPD & 0.69 & 9.725 & $<1 \mathrm{E}-4$ & 0.30 & \\
\hline 001298 & 100.05850 & 9.34126 & $\mathrm{w}$ & 223968804 & QPS & 1.45 & 1.292 & $<1 \mathrm{E}-4$ & 0.23 & 1.295 \\
\hline 001300 & 99.90011 & 9.40728 & $\mathrm{w}$ & 223957908 & $\mathrm{~N}$ & 0.68 & & & & \\
\hline 001302 & 99.81543 & 9.49149 & w & 223951822 & $\mathrm{~N}$ & 0.68 & & & & \\
\hline 001303 & 100.01678 & 9.45196 & W & 223965989 & MP? & 0.64 & 0.821 & $<1 \mathrm{E}-4$ & & 0.819 \\
\hline 001304 & 100.05672 & 9.41372 & $\mathrm{c}$ & 223968688 & $\mathrm{Be}$ & 2.17 & 1.081 & $<1 \mathrm{E}-4$ & & 1.117 \\
\hline 001304 & 100.05672 & 9.41372 & $\mathrm{c}$ & 223968688 & $\mathrm{Be}$ & 2.17 & 1.127 & $<1 \mathrm{E}-4$ & & "" \\
\hline 001306 & 99.86619 & 9.47752 & & 223955517 & $\mathrm{U}$ & & & & & \\
\hline 001307 & 99.84556 & 9.60470 & $\mathrm{w}$ & 223954040 & QPS & 1.17 & 9.585 & $<1 \mathrm{E}-4$ & 0.06 & 9.684 \\
\hline 001308 & 99.99685 & 9.45679 & $\mathrm{c}$ & 223964667 & QPD & 0.63 & 6.717 & $<1 \mathrm{E}-4$ & 0.40 & 6.456 \\
\hline 001309 & 99.92771 & 9.53093 & w & 223959949 & $\mathrm{~N}$ & 0.45 & & & & \\
\hline 001310 & 99.87232 & 9.34970 & w & 223955994 & $\mathrm{U}$ & 1.29 & & & & \\
\hline 001312 & 99.86491 & 9.38590 & & 223955438 & $\mathrm{~N}$ & & & & & \\
\hline 001313 & 100.15139 & 9.31597 & w & 223974593 & $\mathrm{Be}$ & 0.45 & 1.156 & $<1 \mathrm{E}-4$ & 0.28 & 1.156 \\
\hline 001313 & 100.15139 & 9.31597 & w & 223974593 & $\mathrm{Be}$ & 0.45 & 0.906 & $<1 \mathrm{E}-4$ & & "" \\
\hline 001359 & 100.27631 & 9.49189 & w & 223982169 & QPS & 0.29 & 3.181 & $<1 \mathrm{E}-4$ & -0.17 & 3.162 \\
\hline 001386 & 99.82098 & 9.97093 & W & 223952236 & $\mathrm{~N}$ & 1.20 & & & & \\
\hline 001388 & 99.88739 & 9.94156 & w & 223957004 & $\mathrm{~N}$ & 1.10 & & & & \\
\hline
\end{tabular}


Table F.1. continued.

\begin{tabular}{|c|c|c|c|c|c|c|c|c|c|c|}
\hline CSIMon-\# & $\mathrm{RA}^{1}$ & $\operatorname{Dec}^{1}$ & Class $^{2}$ & CoRoT_2011 & $\mathrm{LC}^{3}$ & $M_{\star}\left(M_{\odot}\right)$ & $P_{2011}^{\mathrm{CoRoT}}(\mathrm{d})$ & $\mathrm{FAP}^{4}$ & $\mathrm{Q}^{5}$ & $P_{2008}^{\mathrm{CoRoT}}(\mathrm{d})^{6}$ \\
\hline 001389 & 99.95082 & 9.98490 & & 223961560 & $\mathrm{U}$ & & & & & \\
\hline 001573 & 100.05239 & 10.09457 & $\mathrm{c}$ & 223968439 & $\mathrm{~S}$ & 0.62 & & & & 8.688 \\
\hline 001579 & 100.14630 & 10.07272 & w & 223974272 & QPS & 0.69 & 1.34 & $<1 \mathrm{E}-4$ & 0.23 & - \\
\hline 001581 & 100.39892 & 10.07105 & $\mathrm{~W}$ & 602103885 & & 0.27 & & & & - \\
\hline 001588 & 100.37020 & 10.15404 & & 223988020 & $\mathrm{U}$ & & & & & \\
\hline 001590 & 100.41213 & 10.15986 & & 605537061 & $\mathrm{P}$ & & 3.175 & $<1 \mathrm{E}-4$ & 0.36 & - \\
\hline 001594 & 100.07184 & 10.22646 & & 223969672 & $\mathrm{Be}$ & & 0.800 & $<1 \mathrm{E}-4$ & & \\
\hline 001594 & 100.07184 & 10.22646 & & 223969672 & $\mathrm{Be}$ & & 0.902 & $<1 \mathrm{E}-4$ & & \\
\hline 001596 & 100.28734 & 10.23947 & & 223982807 & $\mathrm{~N}$ & & & & & \\
\hline 001597 & 100.25637 & 10.24891 & & 223980941 & $\mathrm{U}$ & & 3.819 & $<1 \mathrm{E}-4$ & 0.50 & 3.794 \\
\hline 001598 & 100.35009 & 10.24228 & $\mathrm{c}$ & 602113781 & $\mathrm{U}$ & & & & & - \\
\hline 001599 & 100.06796 & 10.31211 & $\mathrm{c}$ & 616735324 & $\mathrm{U}$ & & 0.946 & $6.0 \mathrm{E}-3$ & - & - \\
\hline 001610 & 99.97960 & 9.36463 & w & 602075320 & $\mathrm{~N}$ & 0.44 & & & & - \\
\hline 001612 & 100.24800 & 9.49767 & $\mathrm{c}$ & 616943997 & & 0.30 & & & & 9.34 \\
\hline 001618 & 100.44000 & 9.65865 & w & 603408592 & & 0.30 & & & & - \\
\hline 001627 & 100.28042 & 10.22539 & & 223982423 & & & & & & 9.026 \\
\hline 001628 & 100.17600 & 9.81436 & & 605538554 & $\mathrm{~N}$ & & & & & - \\
\hline 005009 & 100.54130 & 9.79835 & $\mathrm{c}$ & 602091907 & $\mathrm{U}$ & 0.32 & & & & - \\
\hline 005589 & 100.16910 & 9.46370 & w & 603396398 & & 0.30 & 12.519 & $<1 \mathrm{E}-4$ & $\sim 0$ & - \\
\hline 005664 & 100.22704 & 9.15886 & $\mathrm{c}$ & 223979150 & QP & 0.45 & 1.192 & $<1 \mathrm{E}-4$ & $<0$ & - \\
\hline 005745 & 100.51808 & 9.16136 & $\mathrm{c}$ & 617022483 & & 0.32 & 15.946 & $<1 \mathrm{E}-4$ & & - \\
\hline 005836 & 100.37126 & 9.30428 & $\mathrm{c}$ & 602075360 & & 0.34 & & & & - \\
\hline 006037 & 99.87232 & 9.72772 & w & 223955976 & & 0.45 & 3.261 & $<1 \mathrm{E}-4$ & 0.16 & - \\
\hline 006079 & 99.87131 & 9.71071 & $\mathrm{c}$ & 223955882 & EB & 0.45 & 0.511 & $<1 \mathrm{E}-4$ & 0.05 & - \\
\hline 006324 & 99.95312 & 9.29311 & $\mathrm{c}$ & 616996779 & & 0.36 & & & & - \\
\hline 006325 & 100.06033 & 9.22703 & $\mathrm{c}$ & 616996720 & & 0.54 & 0.956 & $7.0 \mathrm{E}-3$ & - & - \\
\hline 006465 & 99.85485 & 9.54393 & $\mathrm{c}$ & 223954720 & EB & 0.88 & 2.829 & $9.0 \mathrm{E}-1^{b}$ & 0.29 & - \\
\hline $006491^{*}$ & 99.85626 & 9.52761 & $\mathrm{c}$ & 616920065 & $\mathrm{Be}$ ? & 1.08 & 2.452 & $3.0 \mathrm{E}-4$ & & - \\
\hline $006491^{*}$ & 99.85626 & 9.52761 & $\mathrm{c}$ & 616920065 & $\mathrm{Be} ?$ & 1.08 & 2.75 & $5.0 \mathrm{E}-4$ & & - \\
\hline $006491^{*}$ & 99.85626 & 9.52761 & $\mathrm{c}$ & 616920065 & $\mathrm{Be} ?$ & 1.08 & 2.271 & $2.5 \mathrm{E}-3$ & & - \\
\hline 006930 & 99.76704 & 9.27055 & $\mathrm{c}$ & 223948224 & & 0.65 & & & & - \\
\hline 006991 & 99.84170 & 10.10648 & $\mathrm{c}$ & 223953770 & & 0.53 & & & & - \\
\hline 007004 & 99.85813 & 10.08542 & $\mathrm{c}$ & 223954942 & & 0.80 & & & & - \\
\hline $014132^{12}$ & 99.76481 & 9.27110 & $\mathrm{c}$ & 602070634 & QPD & 0.28 & 9.102 & $<1 \mathrm{E}-4$ & 0.24 & - \\
\hline
\end{tabular}

